\title{
UNIVERSAL DISPERSION TABLES \\ 1. LOVE WAVES ACROSS OCEANS AND CONTINENTS ON A SPHERICAL EARTH
}

\author{
By DON L. ANDERson
}

\begin{abstract}
The variational approach to surface wave dispersion problems has been largely replaced by the powerful method of Haskell which is exact and particularly convenient for use on digital computers. This paper shows how the two methods may be combined to yield dispersion curves which can be used to interpret data from any layered structure.

A set of graphs and tables is presented which can be used to calculate the dispersion of Love waves in the period range of 4 to 1000 seconds over any spherical earth model. In addition, it is possible to determine by inspection which portion of the earth is contributing to a set of observed dispersion data thereby facilitating the design of an appropriate earth model. These tables can be used to determine how much freedom can be taken with proposed models without violating dispersion data. Application to the inverse problem is immediate.
\end{abstract}

\section{INTRODUCTION}

The calculation of the dispersion relationships for surface waves travelling over realistic spherical earth models is a fundamental seismological problem, arising both in earth structure and in source mechanism studies. It is a routine matter now, for those having access to high speed computers, to calculate the dispersion appropriate for any mode of propagation in a given radially symmetric earth model. Fairly complete fundamental mode solutions have been published for the standard earth models of Gutenberg, Jeffreys, Lehmann, Bullen and Bullard, and minor variations of these velocity and density structures. Comparison of these theoretical calculations with long period observational data show that the Gutenberg or Lehmann velocity structures and the Bullen A density structures provide better agreement than the alternatives, but none of the standard earth models is completely satisfactory. Because of the large number of parameters involved in defining a realistic earth model and the complicated way in which they enter into the dispersion relations, it is difficult to determine which parameters must be changed in order to achieve better agreement between the theory and the data. Even in crustal studies where the total thickness of the earth's crust is the only quantity of interst, good agreement between observed dispersion and a curve from a set of standard curves where only the total crustal thickness is varied is seldom obtained. This indicates that the velocity-density structure of the standard model must also be varied. Published dispersion curves or families of dispersion eurves do not permit the estimation of the effect of these variations. A large volume of dispersion curve families would be required to extend the standard curve matching method to include the effects of crustal and mantle layering and different crustal velocitydensity structures.

The trial and error method of modifying a test structure and recomputing dispersion is a slowly converging process without clear-cut guide lines as to the necessary modifications. Even with a high-speed digital computer the calculations are so tedious that only the most tentative efforts have been made to modify a trial struc- 
ture until there is good agreement between theory and data, thereby taking full advantage of the surface wave information. Even in the few cases where good agreement has been obtained between a theoretical dispersion curve and observed dispersion data, it has not been possible to make any statements concerning the possibility of other structures fitting equally well. It is not usually clear which features of the proposed model are necessary and which may be modified without violating the data. It is particularly difficult for other earth scientists to assess the relative merits of various competing earth models.

The present series of papers was motivated by the above considerations but have been expanded to allow groups without access to electronic computers to conveniently compute dispersion for complicated structures by hand or with a desk calculator.

A method is developed here which is a modification of Jeffreys' (1961) suggested use of Rayleigh's principle. Haskell's matrix method is used to calculate the period relationship (phase velocity vs. period) and the eigenfunctions for a layered medium. The eigenfunction is considered invariant for small changes in the physical parameters in the calculation of variational partial derivatives. The result is a series of tables and graphs which show the effect of every parameter in the waveguide at all periods. This information, along with the theoretical dispersion results for the trial model, permits the rapid calculation of the dispersion for any other model by hand or with a desk calculator.

This initial paper presents results pertinent for fundamental Love wave phase velocity calculations in the period range 4 to 1000 seconds. Later papers will deal with Rayleigh waves, higher modes and free oscillations. The tables presented here can also be used to compute Love wave attenuation simply by allowing the parameter perturbations to become complex.

\section{Theory}

Although the variational method has been used to calculate approximately the steady state response of a radially or vertically inhomogeneous waveguide, the method finds its proper role as a corrective scheme to investigate the effect of small changes in the waveguide. It should be pointed out that even though the differences among the various proposed earth models are physically significant, they are mathematically small, and the restriction to "small changes" is not severe.

The analysis follows that of Jeffreys (1961) generalized to multi-layered media. The following analysis also holds for a spherical body with the change of variables given in Anderson and Toksöz (1963). If the horizontal transverse displacement for a wave travelling in the $x$-direction is

$$
v(z) \cos (\omega t-k x)
$$

the average values of the kinetic energy, $T$, and the elastic potential energy, $V$, are

$$
4 T=\int \rho \omega^{2} v^{2} d z, \quad 4 V=\int \mu\left(k^{2} v^{2}+v^{\prime 2}\right) d z
$$


where the integration extends over the entire depth of the waveguide and the prime denotes differentiation with respect to the depth variable $z$.

Since the kinetic energy and the potential energy, averaged over a cycle, are equal

$$
\omega^{2} I_{0}=k^{2} I_{1}+I_{2}
$$

where

$$
\begin{aligned}
& I_{0}=\int \rho v^{2} d z \\
& I_{1}=\int \mu v^{2} d z \\
& I_{2}=\int \mu v^{\prime 2} d z
\end{aligned}
$$

The dispersion relation $\omega=\omega(k)$ for the structure being studied can be obtained by a variety of techniques including numerical integration of the equations of motion, a variational technique or the Thomson-Haskell matrix method. The latter is particularly convenient for use on computers and has the additional advantage, for the problem at hand, of yielding closed form expressions for the above integrals. The theory of the matrix method applied to a variety of surface wave problems is treated by Haskell (1953), Anderson (1961), Anderson (1962), Harkrider and Anderson (1962), Gilbert and MacDonald (1960) and Anderson and Toksöz (1963). It need not be discussed further here. The other methods can also be used as a basis for the sort of calculations presented here, but they would have the disadvantages inherent in any completely numerical method.

Once having the dispersion relation and the eigenfunctions we wish to investigate the change in phase velocity at a given period corresponding to a given change in a physical parameter. If we vary $\mu$, keeping $\omega$ and $\rho$ fixed, we have, to first order,

$$
\frac{\delta c}{\delta \mu}=\left(c k^{2} \frac{\delta I_{1}}{\delta \mu}+c \frac{\delta I_{2}}{\delta \mu}\right) /\left(2 I_{1} k^{2}\right)
$$

Similarly, if we vary $\rho$, keeping $\omega$ and $\mu$ constant

$$
\frac{\delta c}{\delta \rho}=\left(-c^{3} \frac{\delta I_{0}}{\delta \rho}\right) / 2 I_{1}
$$

In a layered structure of $n$ homogeneous layers

$$
\begin{aligned}
& I_{0}=\sum_{m=1}^{n} \int_{h_{m}}^{h_{m+1}} \rho_{m} v^{2} d z=\sum_{m=1}^{n} I_{0_{m}} \\
& I_{1}=\sum_{m=1}^{n} \int_{h_{m}}^{h_{m}+1} \mu_{m} v^{2} d z=\sum_{m=1}^{n} I_{1_{m}} \\
& I_{2}=\sum_{m=1}^{n} \int_{h_{m}}^{h_{m+1}} \mu_{m} v^{\prime 2} d z=\sum_{m=1}^{n} I_{2_{m}}
\end{aligned}
$$


By Rayleigh's Principle, for a perturbation of parameters only in the $m$ th layer

$$
\begin{aligned}
& \delta I_{0}=\delta I_{0_{m}}=\int_{h_{m}}^{h_{m+1}} \delta \rho_{m} v^{2} d z \\
& \delta I_{1}=\delta I_{1_{m}}=\int_{h_{m}}^{h_{m+1}} \delta \mu_{m} v^{2} d z \\
& \delta I_{2}=\delta I_{2_{m}}=\int_{h_{m}}^{h_{m+1}} \delta \mu_{m} v^{\prime 2} d z
\end{aligned}
$$

Because of the linearization the total effect on phase velocity is

$$
d c=\sum_{m=1}^{n}\left(\frac{\delta c}{\delta \mu}\right)_{m} d \mu_{m}+\sum_{m=1}^{n}\left(\frac{\delta c}{\delta \rho}\right)_{m} d \rho_{m}
$$

The unperturbed layers, of course, do not contribute to the summation.

In the Haskell formulation of the surface wave problem the motion-stress vector in the $m$ th layer, $\mathfrak{D}_{m}(z)$, can be written

$$
\mathfrak{D}_{m}(z)=B_{m} \mathfrak{D}_{m}\left(z_{m}\right)
$$

Where $m$ is the layer index and the depth corresponding to $z=z_{m}$ is the top of the $m$ th layer. With the convention

$$
D_{m}(z)=\left(\dot{v} / c, p_{z y}\right)
$$

it follows that

$$
\dot{v}(z) / c=B_{11} \dot{v}\left(z_{m}\right) / c+B_{12} p_{y z}\left(z_{m}\right)
$$

where

$$
\begin{aligned}
B_{11} & =\cos k r_{\beta_{m}} d_{m} \\
B_{12} & =i\left(\mu_{m} r_{\beta_{m}}\right)^{-1} \sin k r_{\beta_{m}} d_{m} \\
d_{m} & =h_{m+1}-h_{m} \\
r_{\beta_{m}} & =\left(c^{2} / \beta_{m}{ }^{2}-1\right)^{1 / 2}
\end{aligned}
$$

The energy integrals can now be calculated exactly

$$
\begin{array}{r}
I_{0_{m}}=-\frac{1}{k^{2}}\left[\left(\frac{\dot{v}}{c}\right)_{m}^{2}\left(\frac{h_{m}}{2}+\frac{\sin 2 Q}{4 k r_{\beta_{m}}}\right)-\left(\frac{\dot{v}}{c}\right)_{m}\left(p_{y z}\right)_{m} \mu_{m}{ }^{-1} r_{\beta_{m}}^{-2}\left(\frac{1-\cos 2 Q}{2 k}\right)\right. \\
\left.+\left(p_{y z}\right)^{2}{ }_{m} \mu_{m}{ }^{-2} r_{\beta_{m}}^{-2}\left(\frac{h_{m}}{2}-\frac{\sin 2 Q}{4 k r_{\beta_{m}}}\right)\right]
\end{array}
$$




$$
\begin{aligned}
& I_{1_{m}}=\frac{\mu_{m}}{\rho_{m}} I_{0_{m}} \\
& I_{2_{m}}=-\left(\frac{\dot{v}}{c}\right)^{2} \mu_{m} r^{2} \beta_{m}^{2}\left[\frac{h_{m}}{2}-\frac{\sin 2 Q}{4 k r_{\beta_{m}}}\right] \\
& -\left(\frac{\dot{v}}{c}\right)_{m}\left(p_{y z}\right)_{m}\left[\frac{1-\cos 2 Q}{2 k}\right]-\left(p_{y z}\right)_{m}^{2} \mu_{m}{ }^{-1}\left[\frac{h_{m}}{2}+\frac{\sin 2 Q}{4 k r_{\beta_{m}}}\right]
\end{aligned}
$$

where $Q=k r_{\beta_{m}} d_{m}$.

The earth stretching transformations of Anderson and Toksöz (1963) were used in the calculation of $I_{0}, I_{1}$, and $I_{2}$, these integrals now representing good approximations for the spherical earth problem.

The effect of other parameters in the system can easily be investigated from the above equations. For example, the following relations are useful

$$
\begin{aligned}
\left(\rho \frac{\delta c}{\delta \rho}\right)_{\beta} & =\left(\rho \frac{\delta c}{\delta \rho}\right)_{\mu}+\left(\mu \frac{\delta c}{\delta \mu}\right)_{\rho} \\
\left(h \frac{\delta c}{\delta h}\right)_{\rho, \mu, \beta} & \approx\left(\rho \frac{\delta c}{\delta \rho}\right)_{\beta} \\
\left(\beta \frac{\delta c}{\delta \beta}\right)_{\rho} & \approx 2\left(\mu \frac{\delta c}{\delta \mu}\right)_{\rho}
\end{aligned}
$$

where $h$ is a layer thickness and the subscripts are quantities being held constant.

\section{Use of Tables and Curves}

The calculation of dispersion over any spherical, layered earth model involves the use of two tables. The first gives the layer parameters for the trial model and the second gives the partial derivatives of phase velocity with respect to these layer parameters at various periods. Complete tables are presented here for two trial earth models - one oceanic and one continental. A series of partial derivative graphs are presented for several models to illustrate their behavior. In addition to the partial derivative tables, a set of tables is given which indicates the effect of a $10 \%$ change in the parameter. The numbers in the partial derivative tables are, in fact, derived from these latter tables.

Table 1 gives the layer index, layer thickness, and depth, shear velocity, density and rigidity for each layer in a model designated Gutenberg-Birch II. This is a continental type model with a density distribution that is closely related to the velocity distribution. Table 2 gives the corresponding values for a model designated CIT13F. This is a model that was designed in the course of a study of the oceanic mantle and gives a good fit to oceanic Love wave data. These models are the trial structures upon which the basic partial derivative tables are based. Tables 1 and 2 also tabulate modifications of these models in which the crust and upper mantle are split into finer layers for detailed studies of this region using shorter period Love 


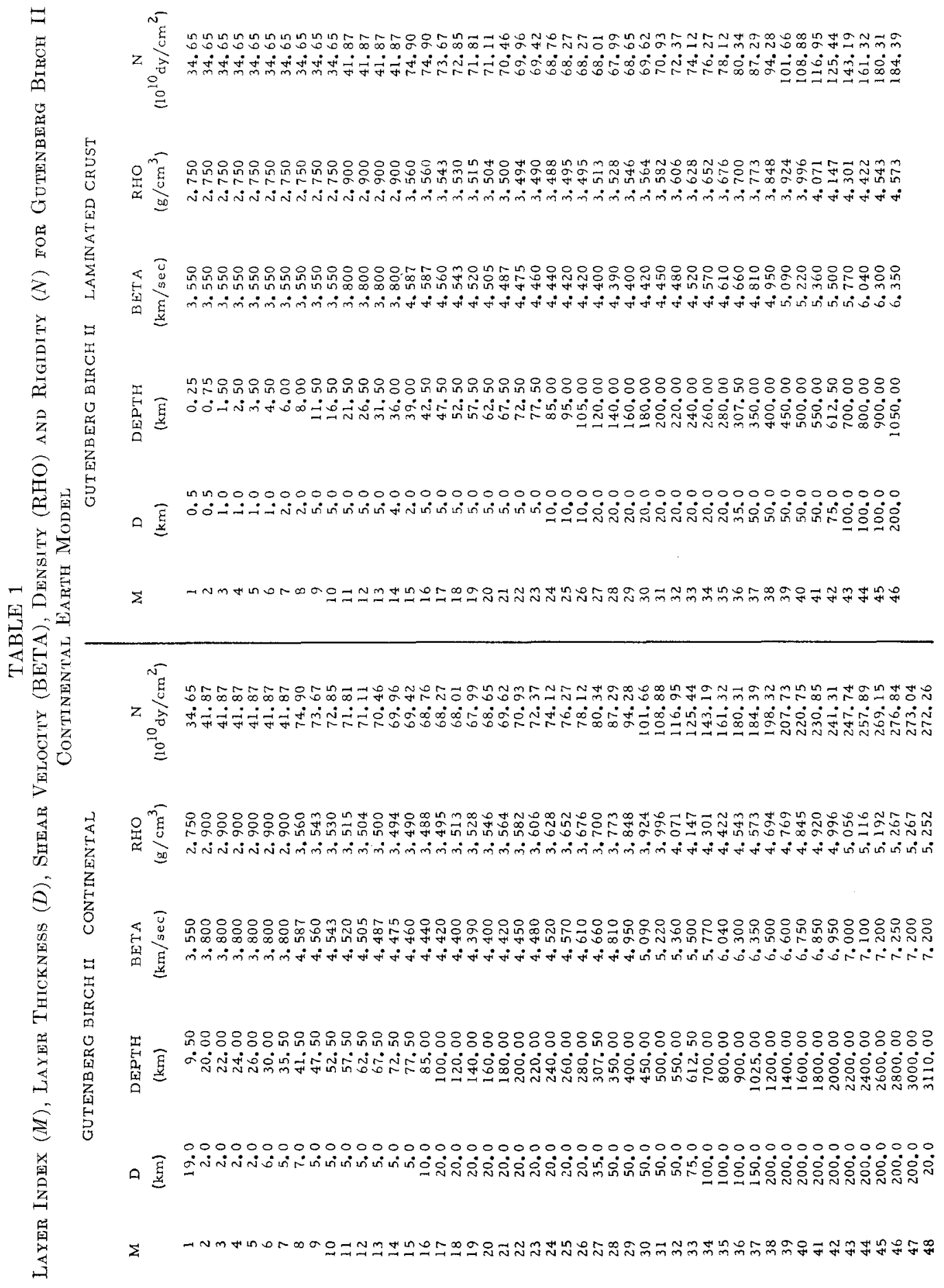




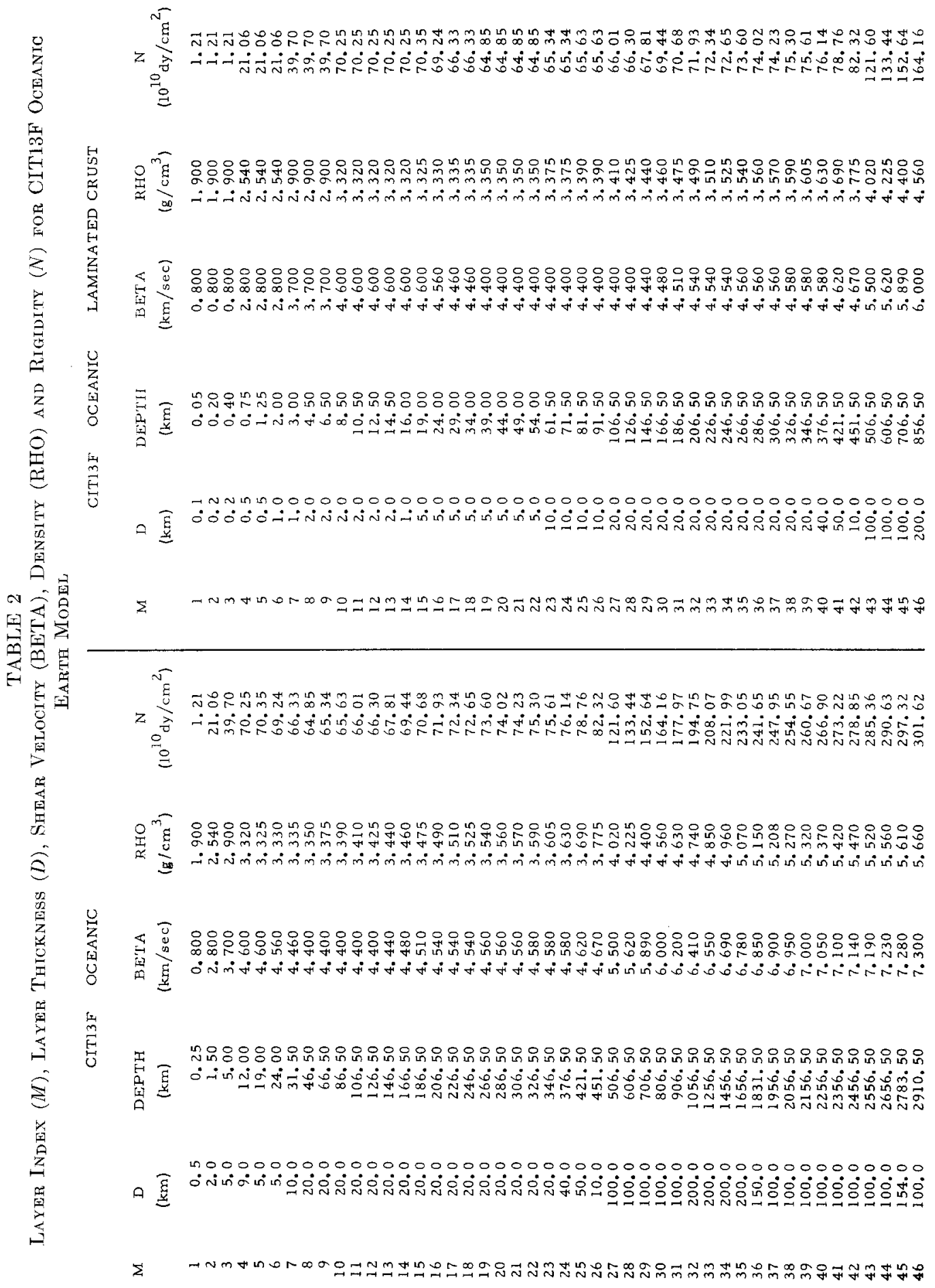


waves. Any given crustal and upper mantle structure can be achieved by perturbing the density and rigidity in the upper layers. Table 3 gives the layer parameters for a model patterned after the Gutenberg IV model of MacDonald and Ness (1961). This model has a Bullen A type density distribution. The Jeffreys-Bullen model is also tabulated in table 3 .

Table 4 presents the complete dispersion and partial derivative results for the Gutenberg-Birch II model. If the parameters in the layers are changed in order to approximate another continental type structure the phase velocity at the indicated period is changed by an amount $\Delta c$, where

$$
\Delta c=\sum_{M=1}^{N} \operatorname{DCDRHO}(M) \cdot \Delta \rho+\sum_{M=1}^{N} \operatorname{DCDMU}(M) \cdot \Delta \mu
$$

In this case $N=46$ is the total number of layers used to approximate the mantle but the summation need only extend over the perturbed layers. DCDRHO and DCDIMU are computerese for $\delta c / \delta \rho$ and $\delta c / \delta \mu$.

Table 5 gives the effect of a ten-percent change in the parameters for this model and in this case

$$
\Delta c=10 \sum_{M=1}^{N}[(\mathrm{DC} / \mathrm{DRHO}(M)) \cdot(\Delta \rho / \rho)]+10 \sum_{M=1}^{N}[(\operatorname{DC} / \mathrm{DMU}(M)) \cdot(\Delta \mu / \mu)]
$$

or

$$
\begin{aligned}
\Delta c=21 \sum_{M=1}^{N}[\mathrm{DC} / \mathrm{DMU}(M)] \cdot \Delta \beta / \beta \\
+10 \sum_{M=1}^{N}[\operatorname{DC} / \mathrm{DMU}(M)+\operatorname{DC} / \mathrm{DRHO}(M)] \cdot \Delta \rho / \rho
\end{aligned}
$$

When only the shear velocity is being perturbed the second summation in the last expression does not contribute. DC/DMU and DC/DRHO are, respectively, $(\rho \delta c / \delta \rho)_{\mu} / 10$ and $(\mu \delta c / \delta \mu)_{\rho} / 10$.

Tables 6 and 7 give the corresponding results for the CIT13F model. As much as possible a new mantle structure should be designed in the framework of the layering given. When this is too restrictive tables 8 through 11 allow finer adjustments to be made in the near surface structure in order to satisfy short period ( $<200$ second) data. Still finer adjustments may be made by splitting the layers again and apportioning the partial derivatives appropriately.

\section{ACCURACy OF THE Method}

The partial derivatives have been tested for a variety of cases, and tables similar to the ones presented here have been in routine use for about 14 months at Caltech. The tables are believed to be error free and very accurate in spite of the various approximations that have been used in their calculation. Each user, however, should convince himself of the reliability and extent of applicability of the tables for his own 
TABLE 3

Layer Parameters for the Jeffreys-Bullen and Gutenberg IV Continental Earth Models

JEFFREYS-BULLEN

$\begin{array}{cc}\text { M } & \text { D } \\ & (\mathrm{km}) \\ 1 & 15 \\ 2 & 18 \\ 3 & 17 \\ 4 & 25 \\ 5 & 35 \\ 6 & 40 \\ 7 & 50 \\ 8 & 50 \\ 9 & 50 \\ 10 & 50 \\ 11 & 63 \\ 12 & 37 \\ 13 & 100 \\ 14 & 100 \\ 15 & 100 \\ 16 & 100 \\ 17 & 150 \\ 18 & 200 \\ 19 & 400 \\ 20 & 400 \\ 21 & 400 \\ 22 & 400\end{array}$

DEPTH
$(\mathrm{km})$
7.5
24.0
41.5
62.5
92.5
130.0
175.0
225.0
275.0
325.0
381.5
431.5
500.0
600.0
700.0
800.0
925.0
1100.0
1400.0
1800.0
2200.0
2600.0

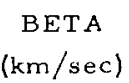

3. 360

3.740

4. 360

4.390

4.440

4.490

4. 560

4. 640

4.720

4.800

4.900

5.040

5.310

5. 660

5.930

6. 130

6.290

6. 440

6. 620

6.830

7.020

7. 210
RHO

$\left(\mathrm{g} / \mathrm{cm}^{3}\right)$

2. 650

2.870
3.330

3. 350

3. 370

3. 410

3.450

3.490

3. 530

3. 570

3.615

3. 700

3.890

4.125

4. 320

4. 490

4.620

4. 739

4.915

5.135

5. 340

5.540

$$
\left(10^{10} \mathrm{dy} / \mathrm{cm}^{2}\right)
$$

29.91

40.14

63. 30

64.56

66.43

68.74

71.73

75.13

78.64

82.25

86.79

93.98

109.68

132.14

151.91

168.72

182.78

196.54

215.39

239. 54

263.15

287.99

\section{GUTENBERG IV}

M

DEPTH
$(\mathrm{km})$
10.5
26.0
41.0
56.0
66.0
76.0
86.0
96.0
111.0
136.0
161.0
186.0
211.0
236.0
276.0
326.0
376.0
426.0
476.0
551.0
651.0
751.0
851.0
951.0
1101.0
1301.0
1501.0
1701.0
1901.0
2051.0
2151.0
2301.0
2501.0
2701.0
2851.0
2951.0

BETA
(km/sec)
3. 55
3.80
4.65
4.60
4.57
4.51
4.46
4.41
4.37
4.35
4.36
4.38
4.42
4.46
4.52
4.66
4.82
5.00
5.14
5.38
5.69
5.96
6.15
6.24
6.34
6.47
6.61
6.72
6.81
6.88
6.88
6.94
7.06
7.14
7.11
7.11

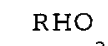

$$
\left(\mathrm{g} / \mathrm{cm}^{3}\right)
$$

2. 738

3.000

3. 321

3. 370

3. 352

3. 358

3. 368

3. 378

3. 388

3. 409

3. 430

3.461

3. 481

3.500

3. 524

3.592

3.650

3.696

3.820

4.010

4. 140

4. 390

4. 550

4.620

4. 730

4. 850

4. 970

5. 050

5. 200

5.302

5. 302

5.439

5.497

5.590

5.697

5. 700

$\mathrm{N}$
$\left(10^{10} \mathrm{dy} / \mathrm{cm}^{2}\right)$
34.50
43.31
71.80
71.30
70.00
68.30
66.99
65.69
64.70
64.50
65.20
66.39
68.00
69.62
71.99
78.00
84.79
92.40
100.92
116.06
134.03
155.93
172.09
179.89
190.12
203.02
217.14
228.04
241.15
250.96
250.96
261.96
273.99
284.97
287.99
288.14




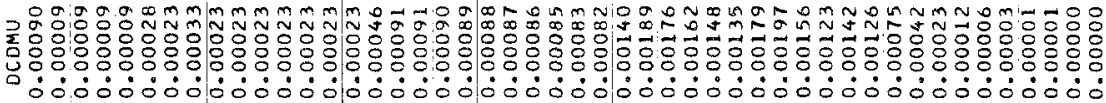

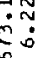

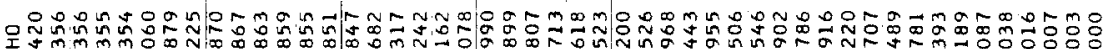

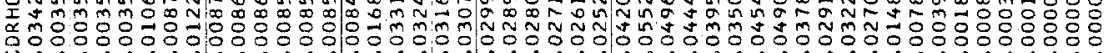

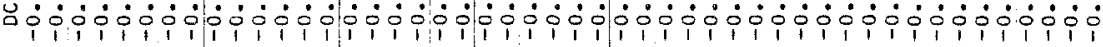

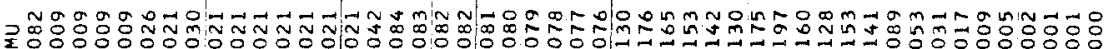

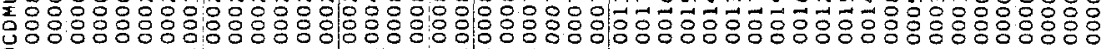

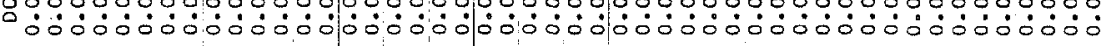

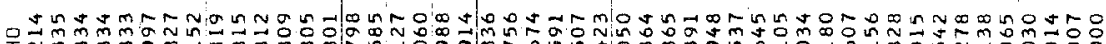

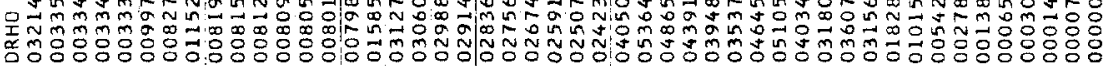

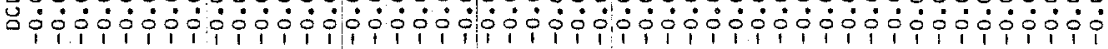

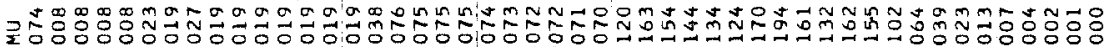

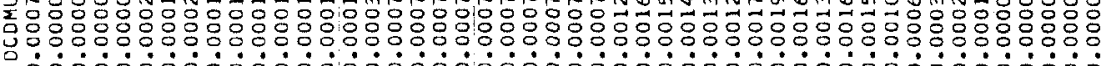

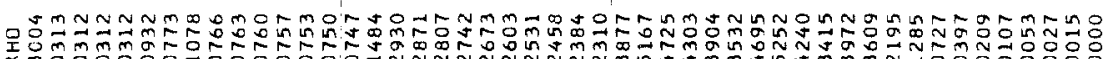

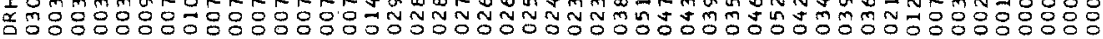

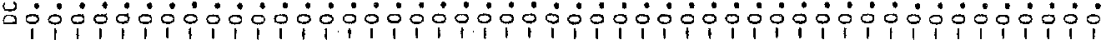

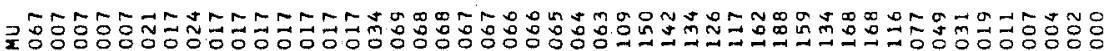

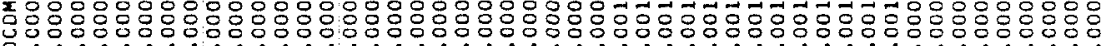

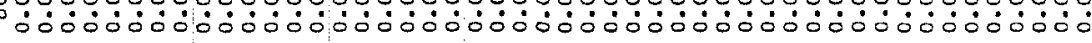

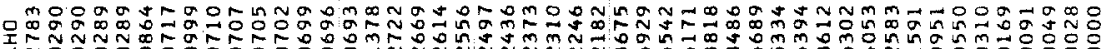

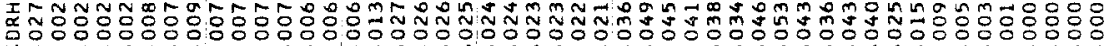

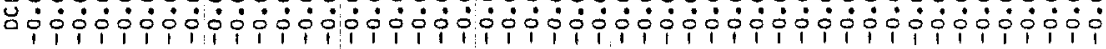

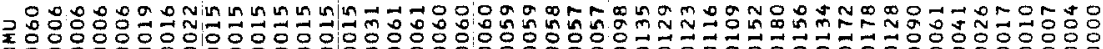

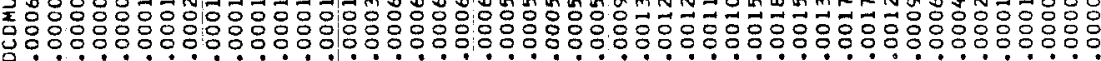
úó்

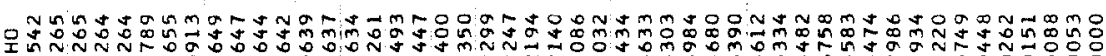

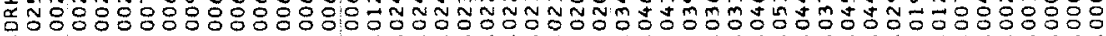
瞒

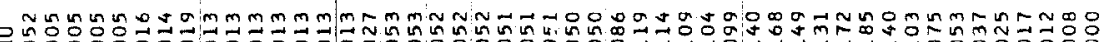

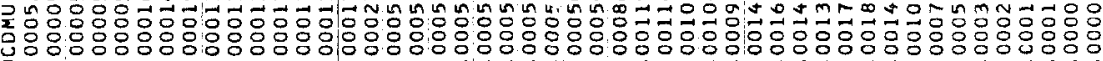

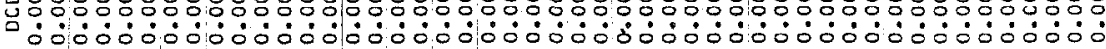

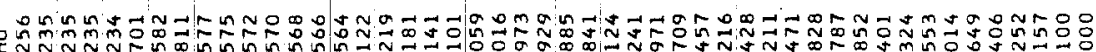

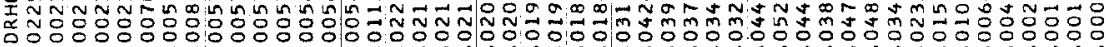

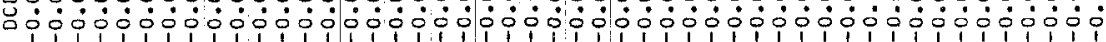

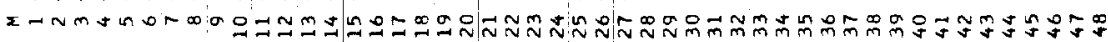




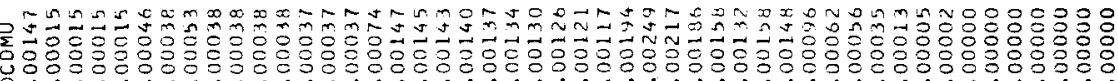

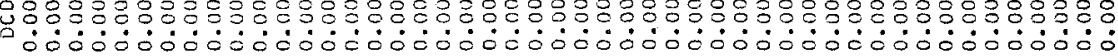

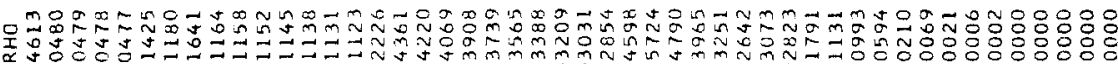

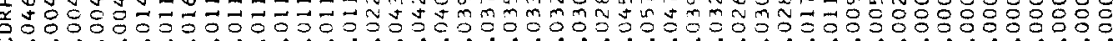

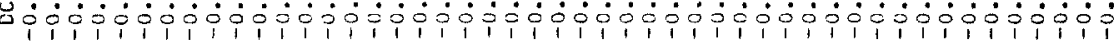

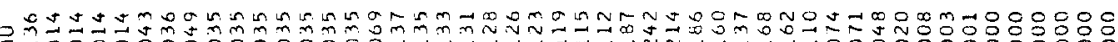

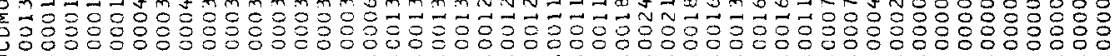

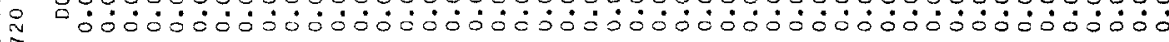

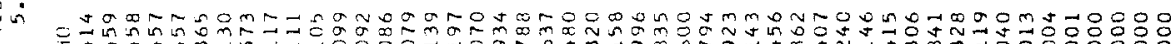

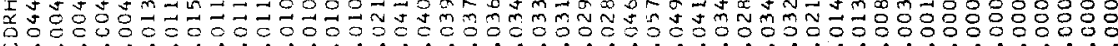

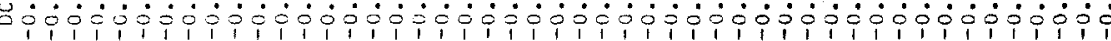

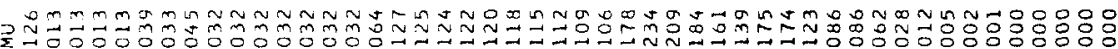

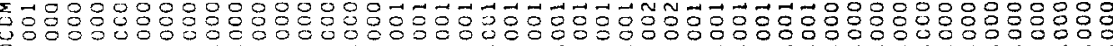

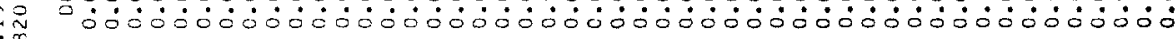

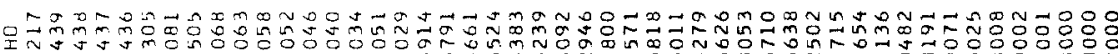

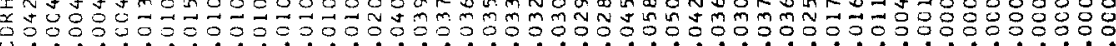

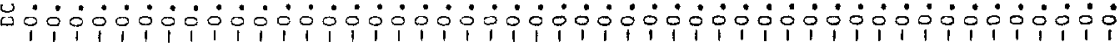

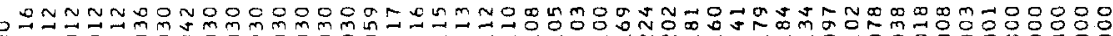

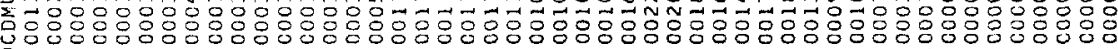

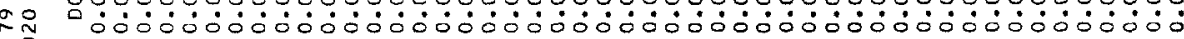
in

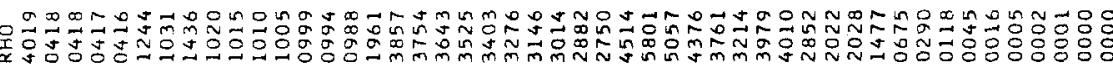

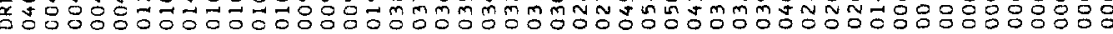

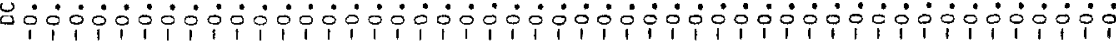

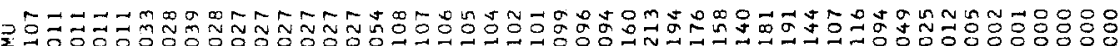

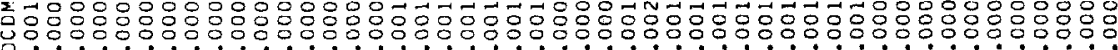

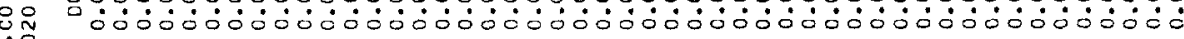
in

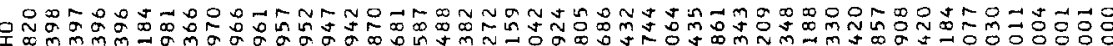

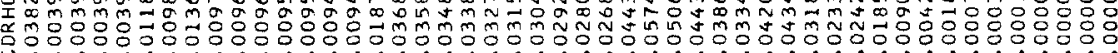

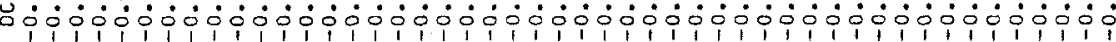

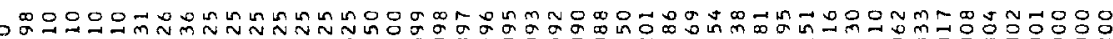

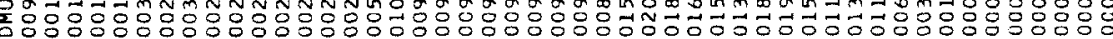

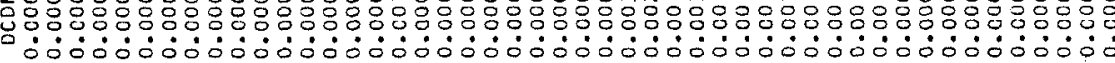

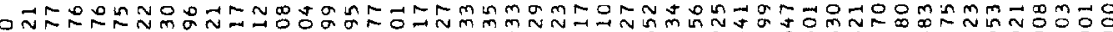

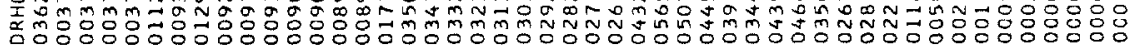

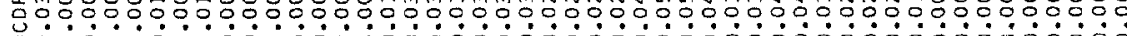

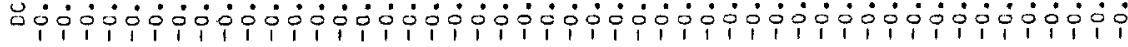

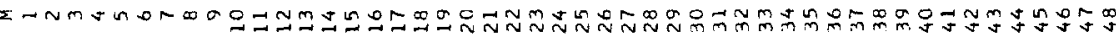


ว ำก M N N

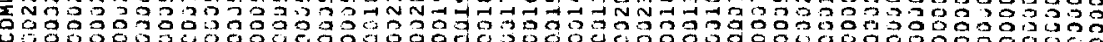

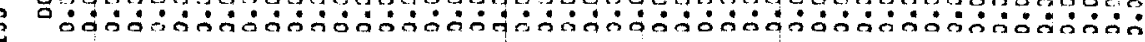

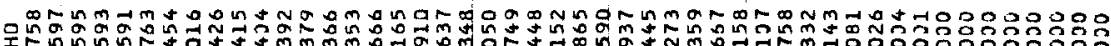

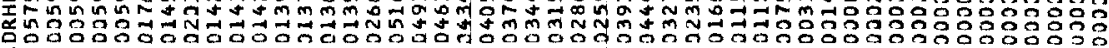

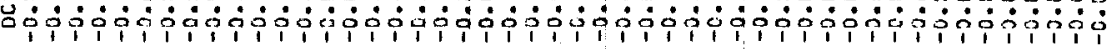

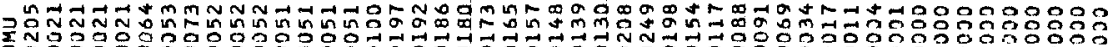

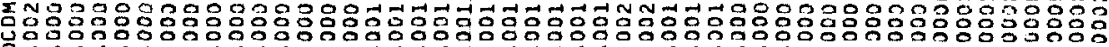

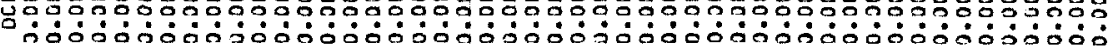

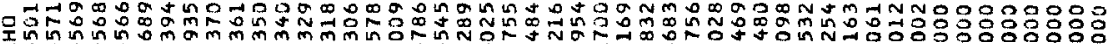

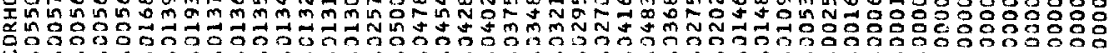

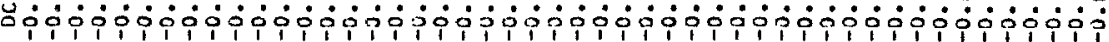

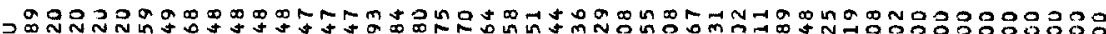

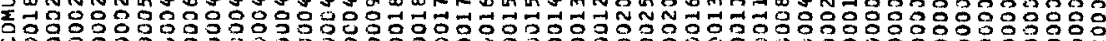

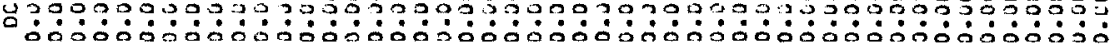

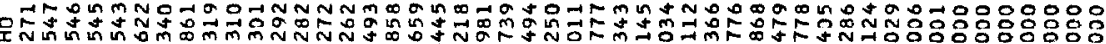

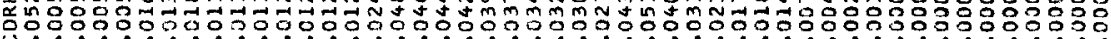

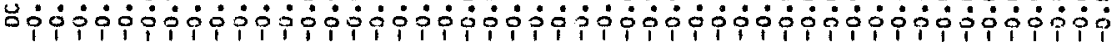

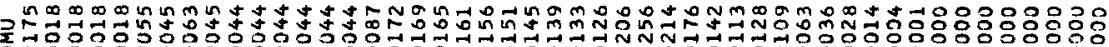

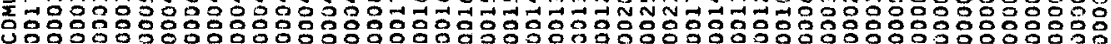

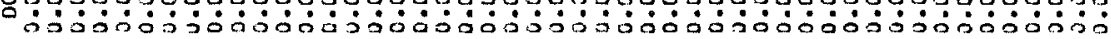

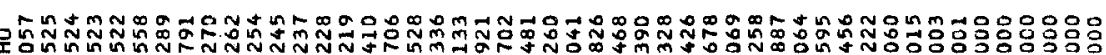

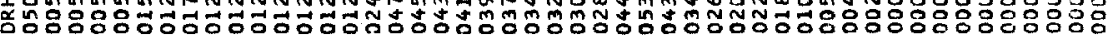

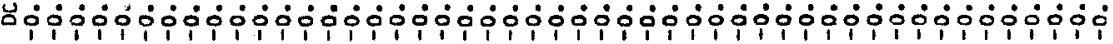

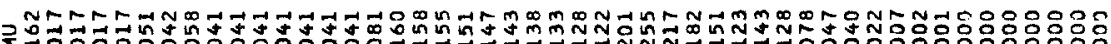

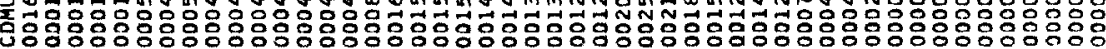

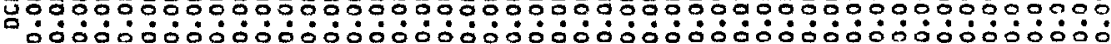

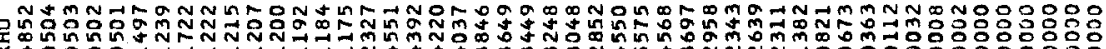

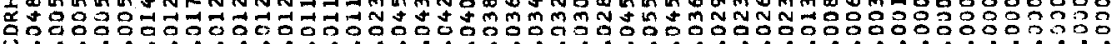

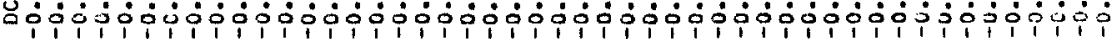

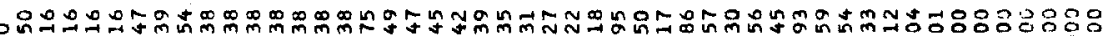

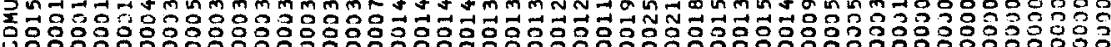

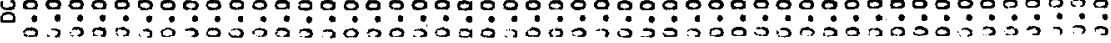

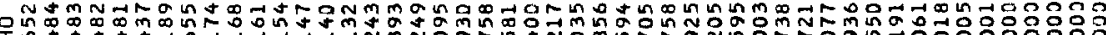

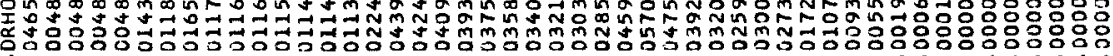

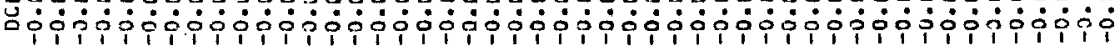

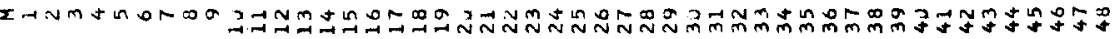


ว

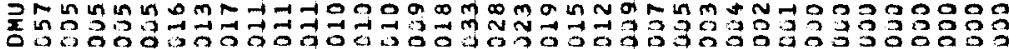

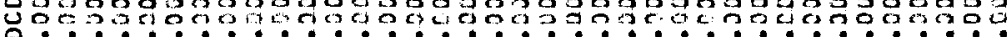

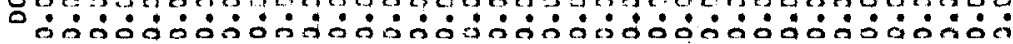

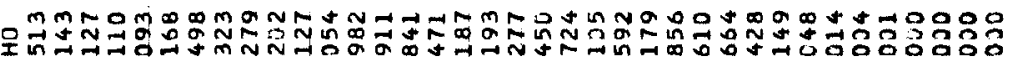

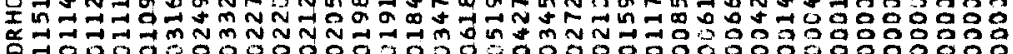

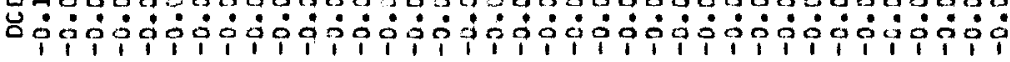

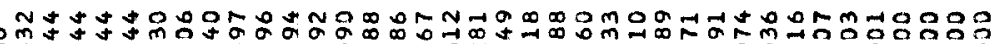

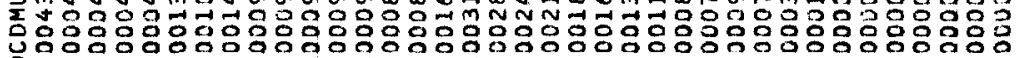

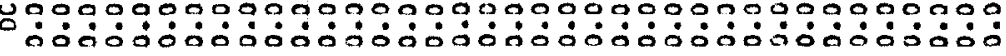

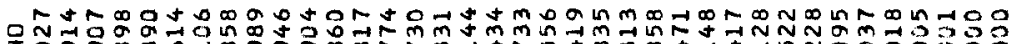

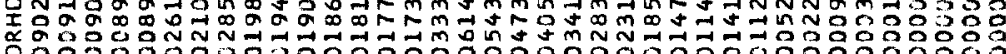

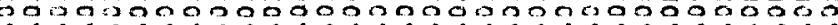

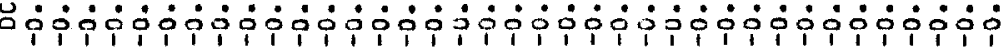

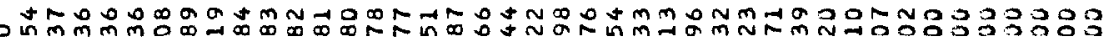

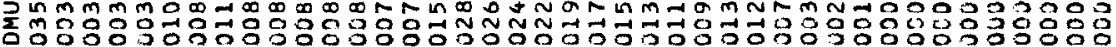

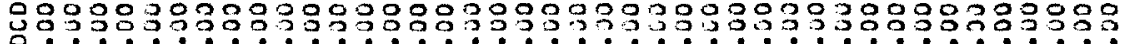

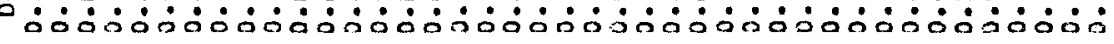

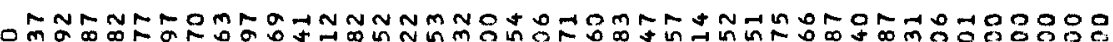

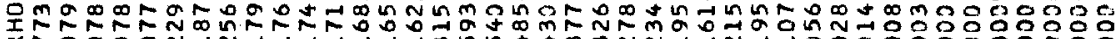

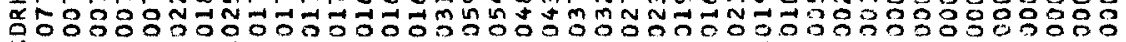

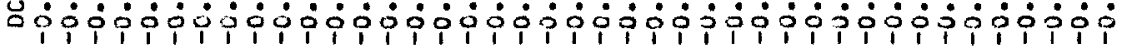
วดิ

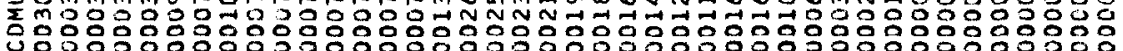

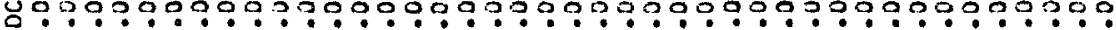

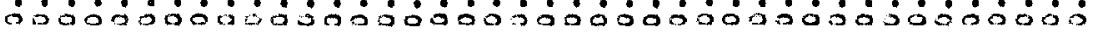

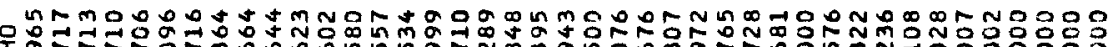

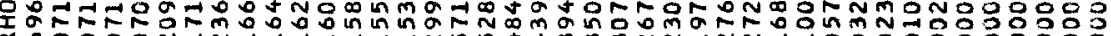

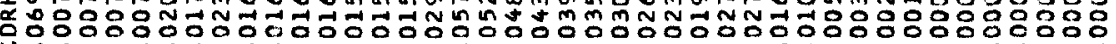

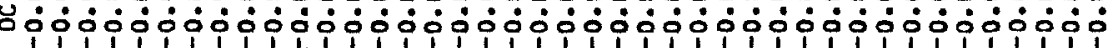

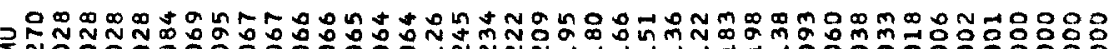

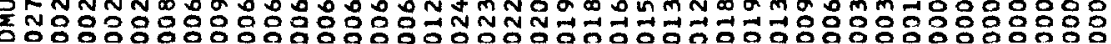

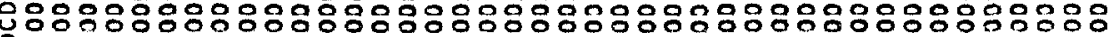

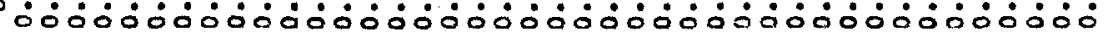

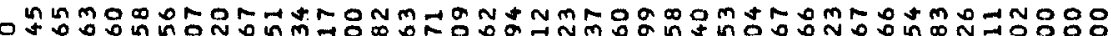

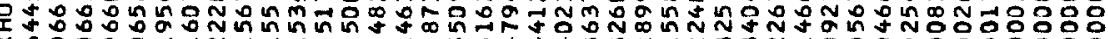

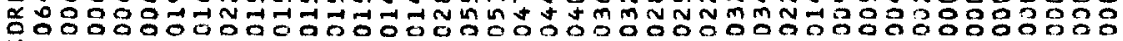

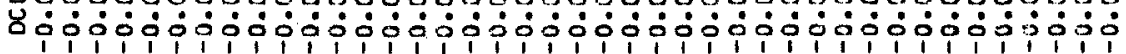

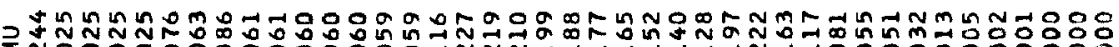

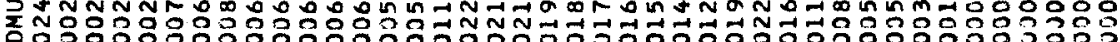

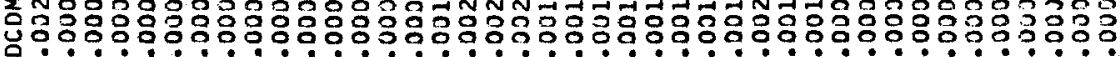

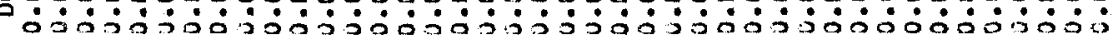

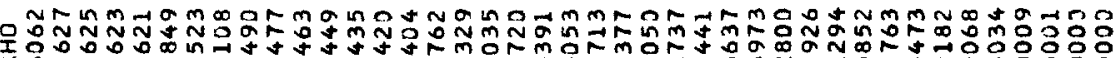

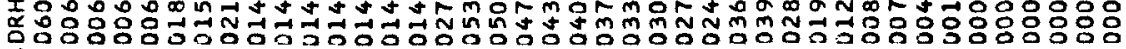

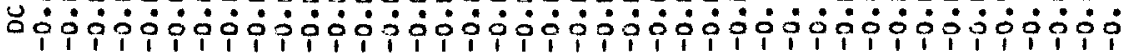

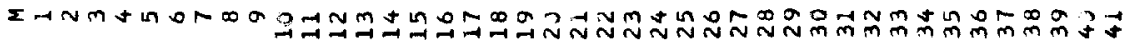




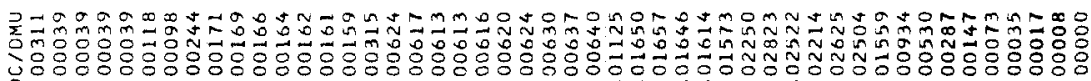

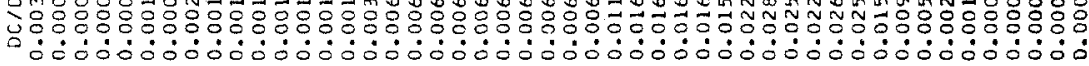

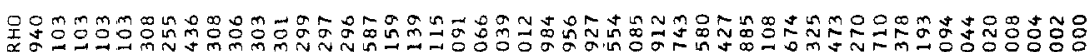

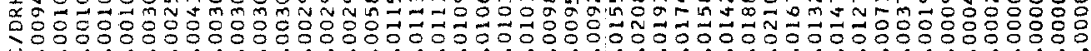

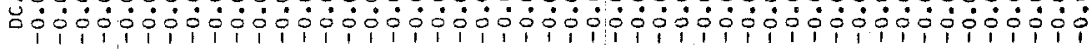

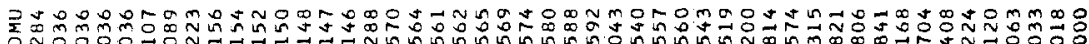

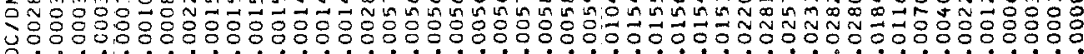

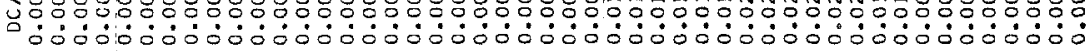

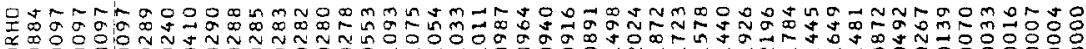

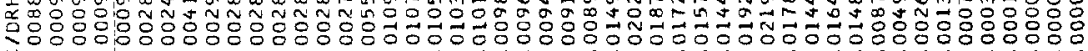

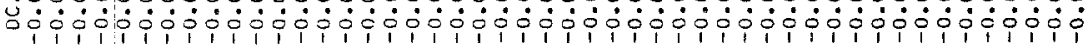

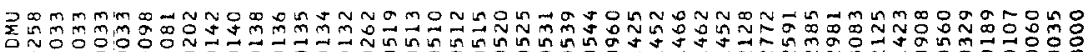

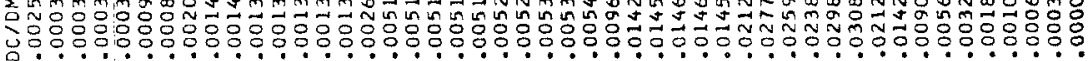

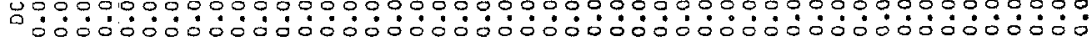

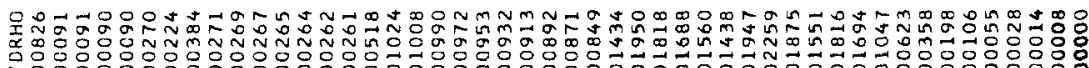

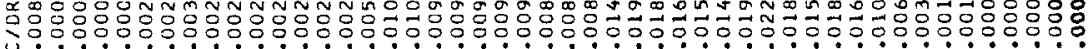

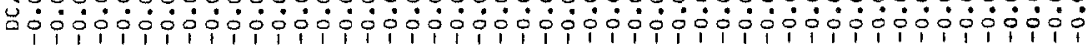

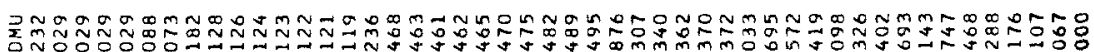

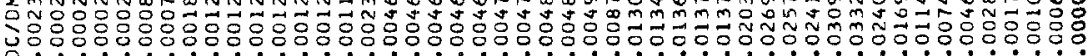

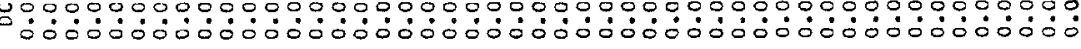

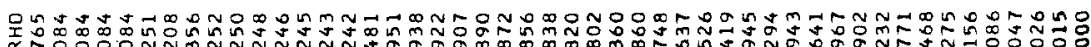

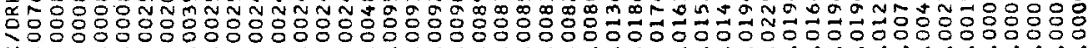

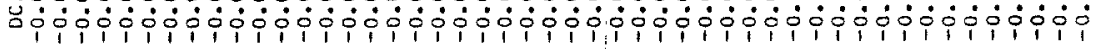

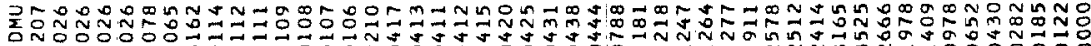

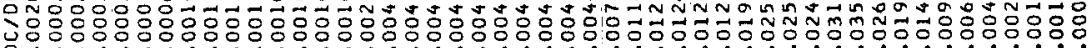

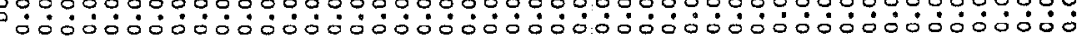

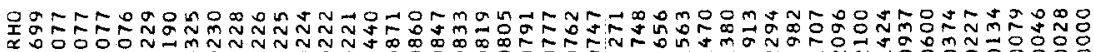
象:

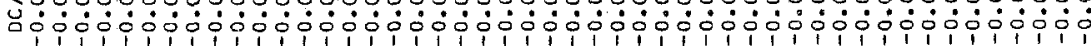

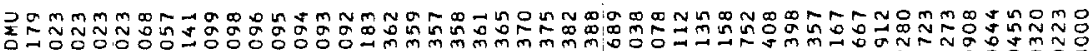

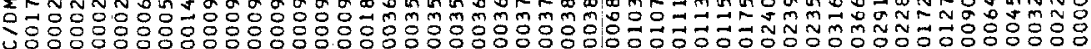

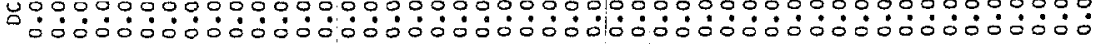

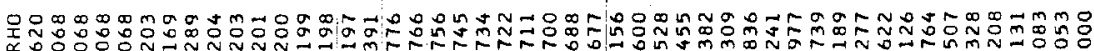

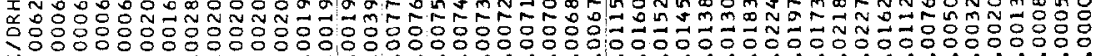

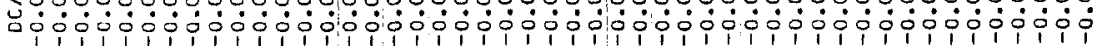

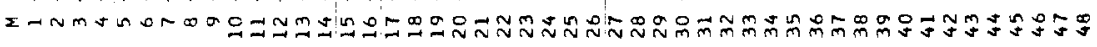




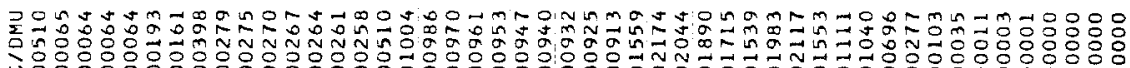

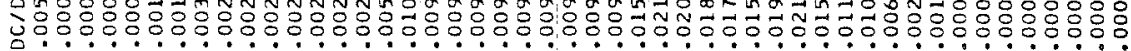

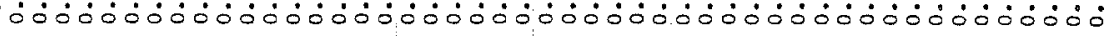

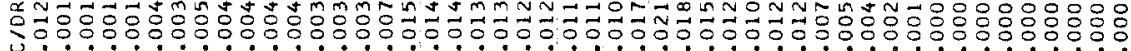

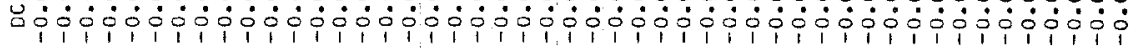

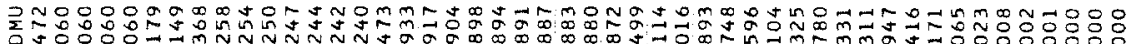

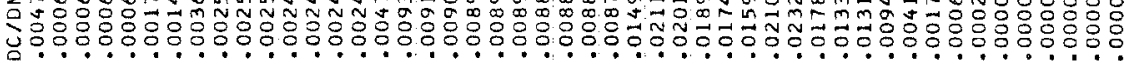

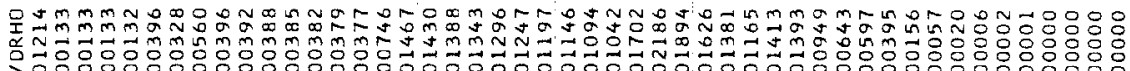

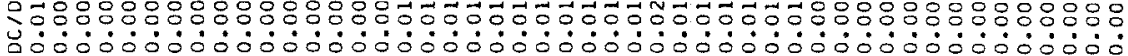

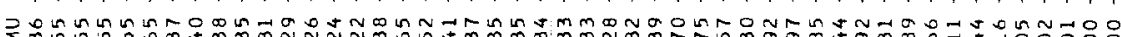

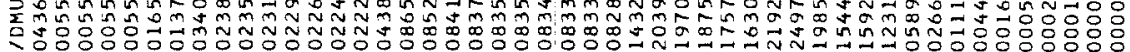
F

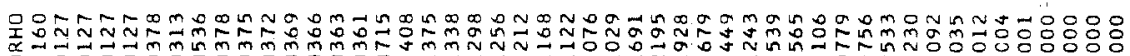

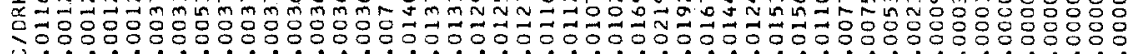

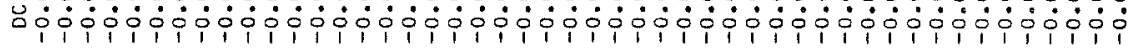

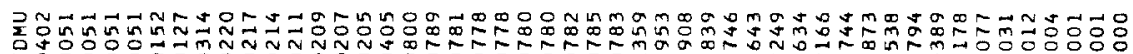

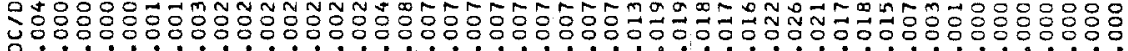

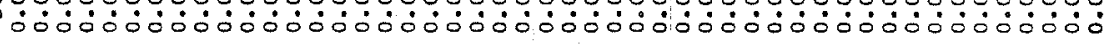

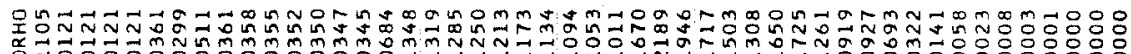
足

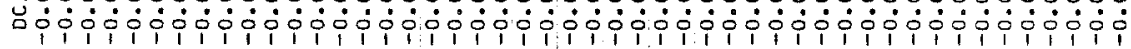

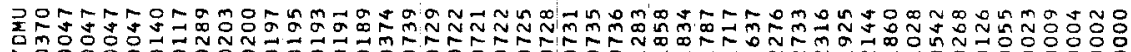

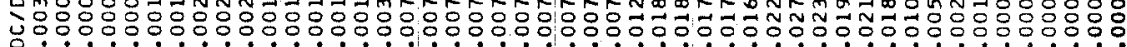

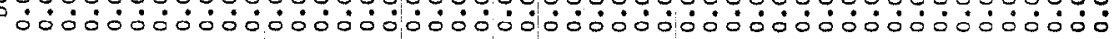

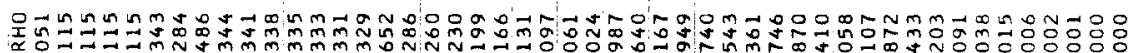

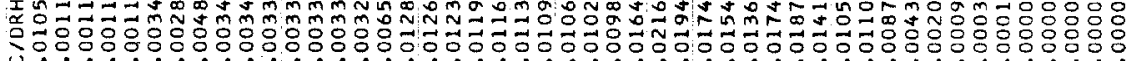

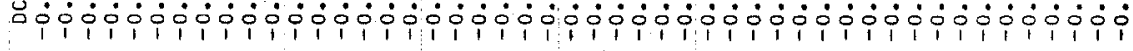

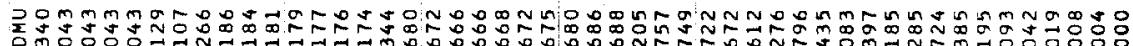

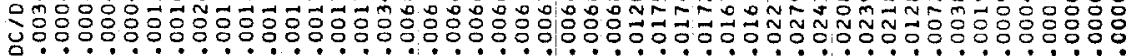

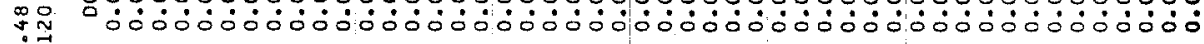

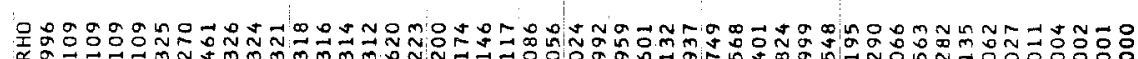

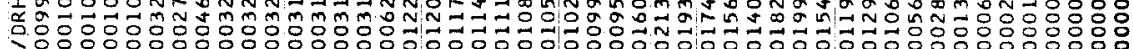
и ú us a 


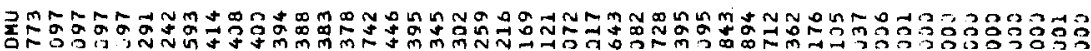

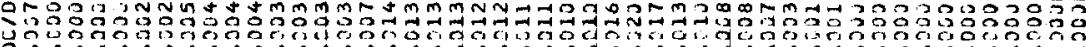
ํㅣㄹ

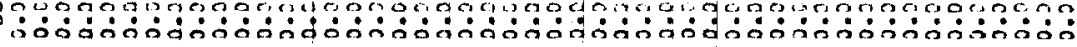

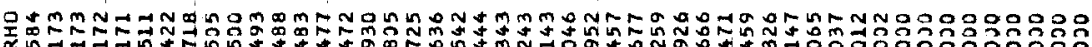

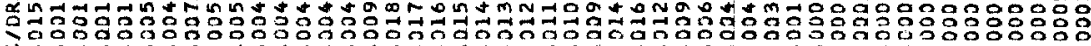

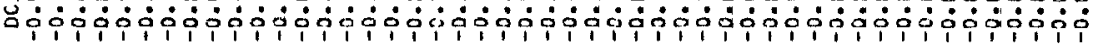

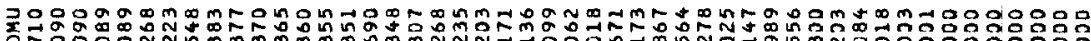

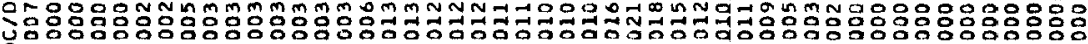

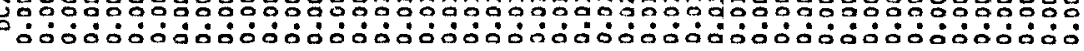

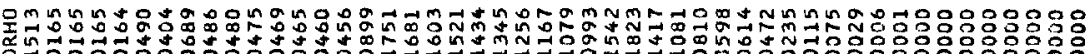

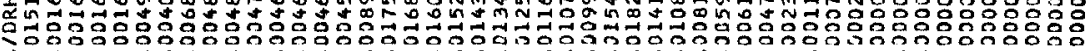

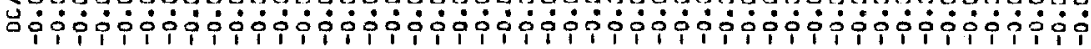

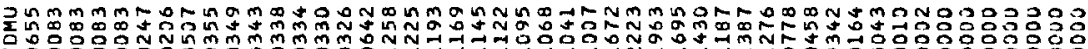

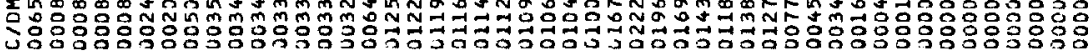

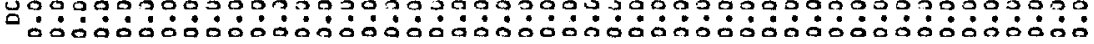

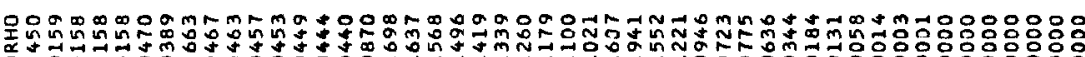

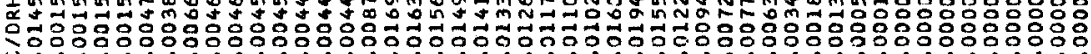

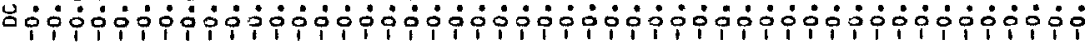

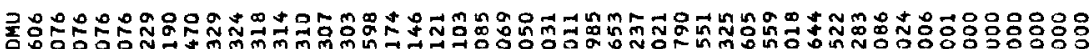

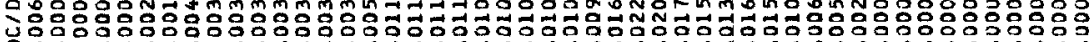

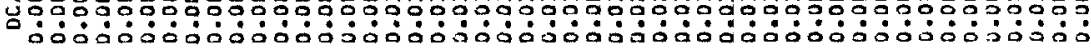

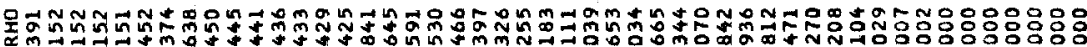

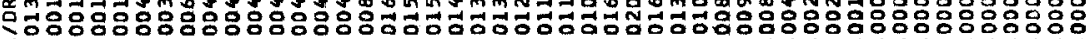

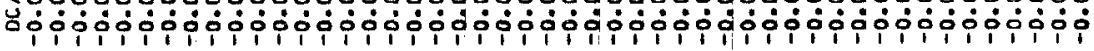

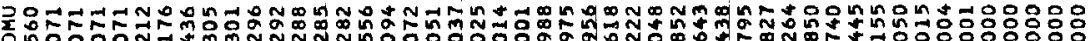

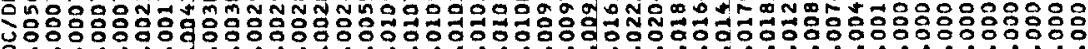

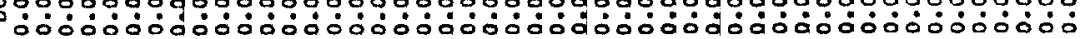

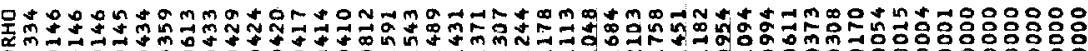

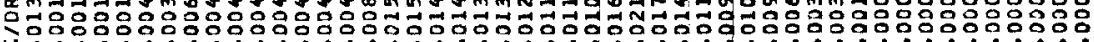

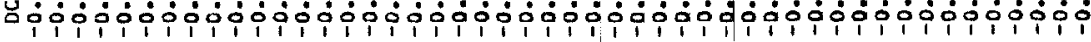

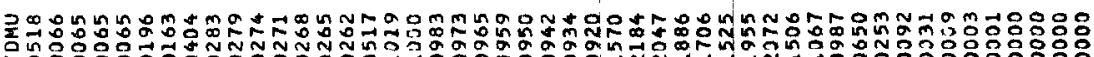

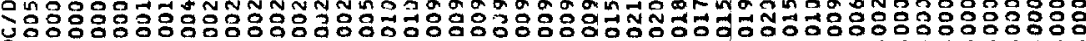

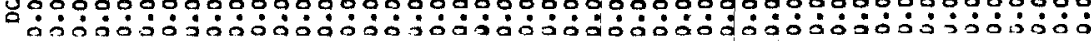

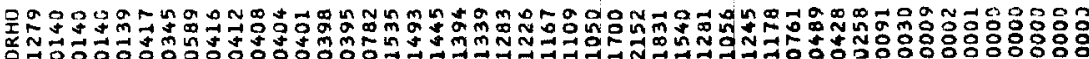

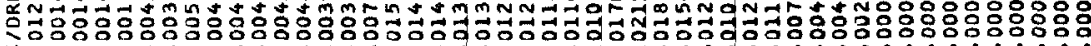

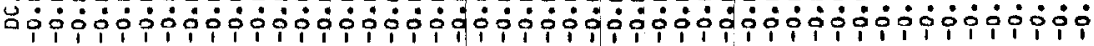

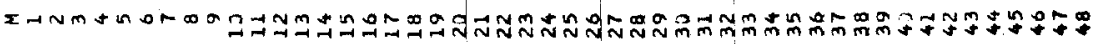




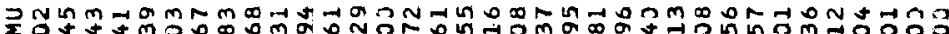

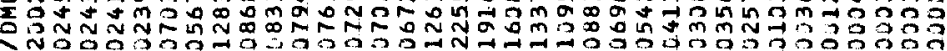

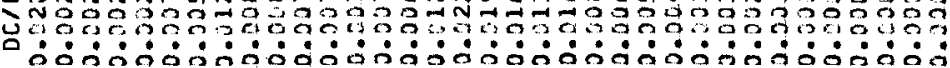
$\dot{\infty}:$

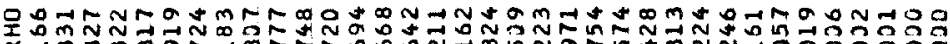
药

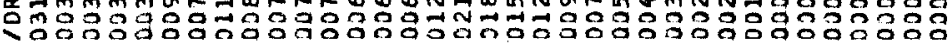

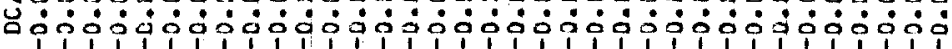

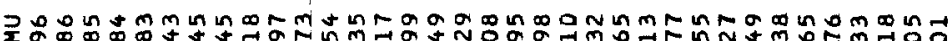

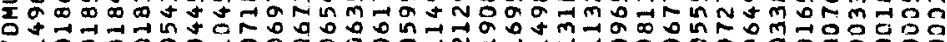

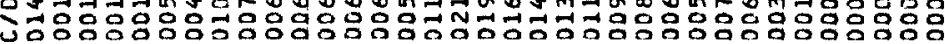

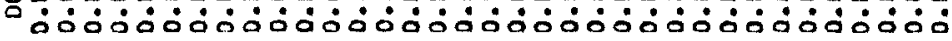

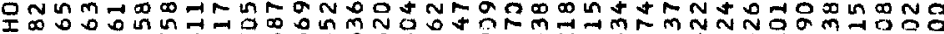

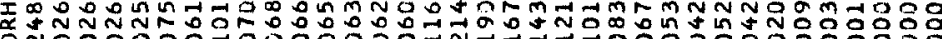

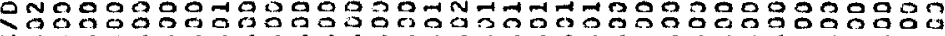

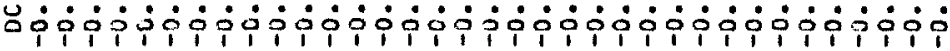

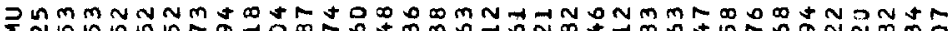

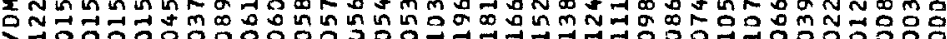

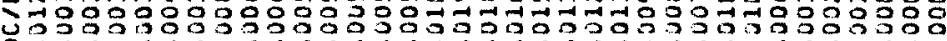
ó.

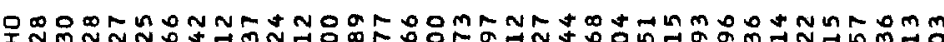

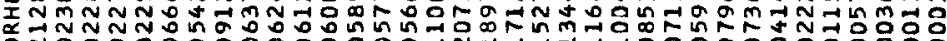

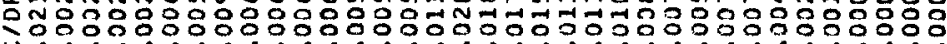

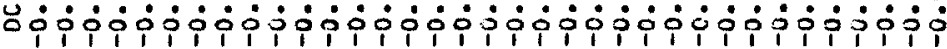

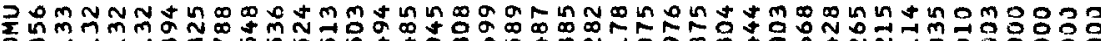

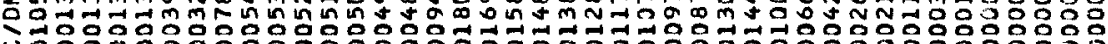

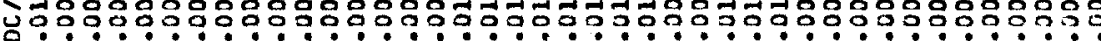

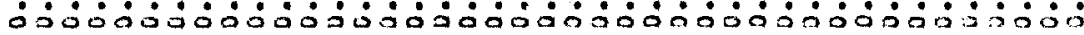

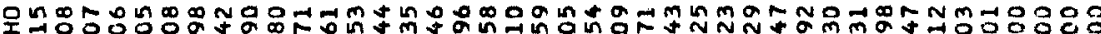

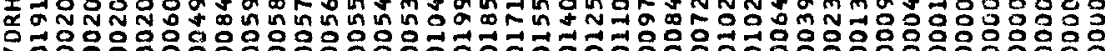

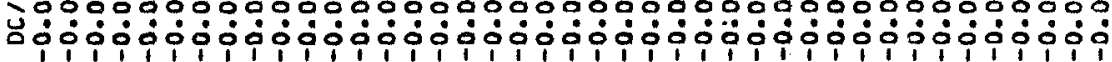
곴

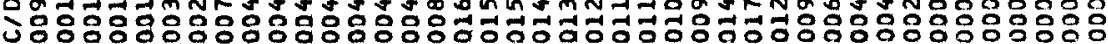

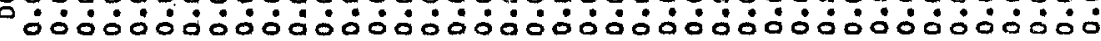

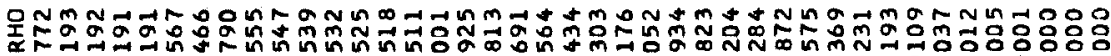

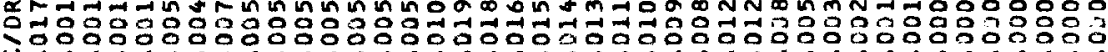

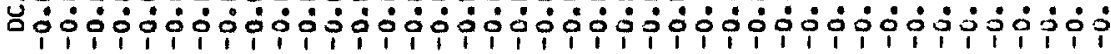

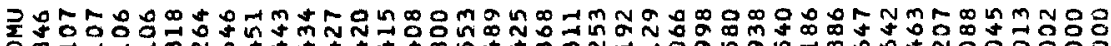

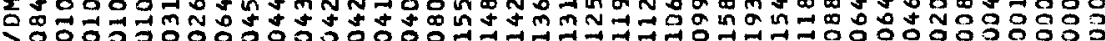

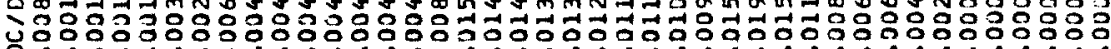

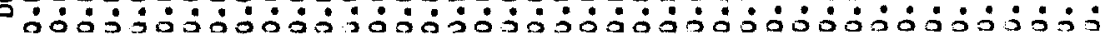

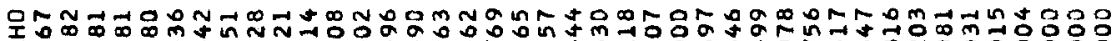

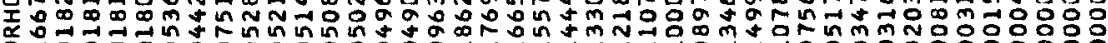

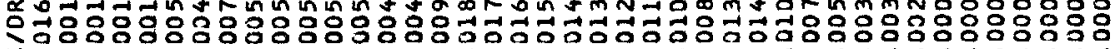

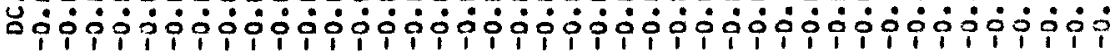




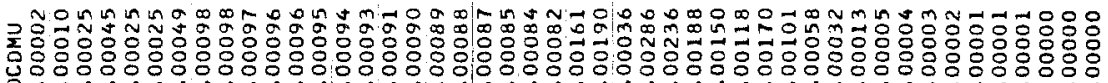

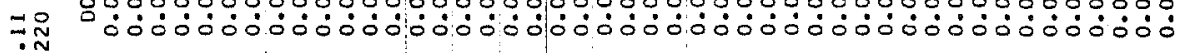
范:

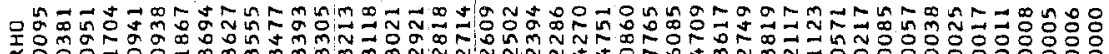

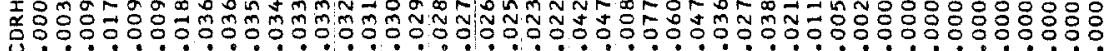

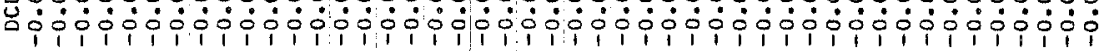

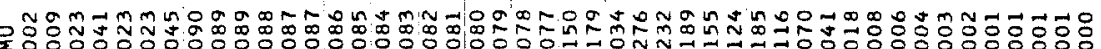

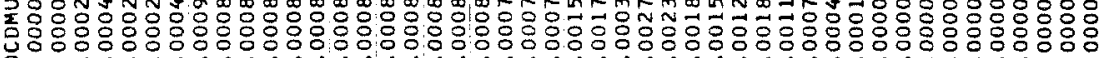

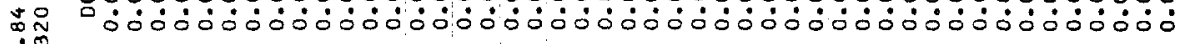
$\stackrel{\dot{0}}{0} \dot{0}$

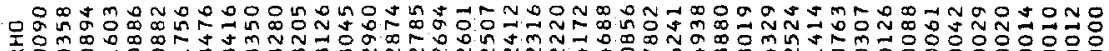

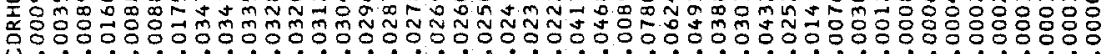

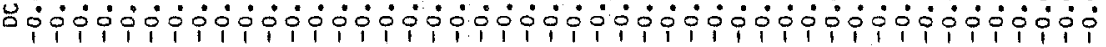

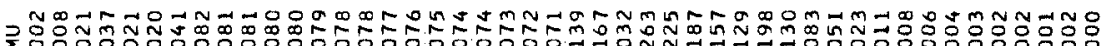

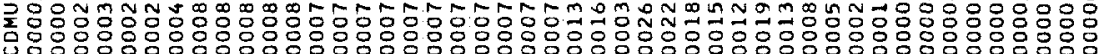

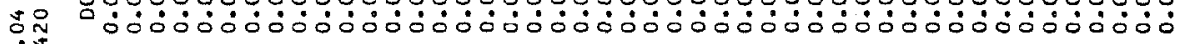
in:

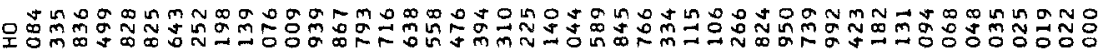

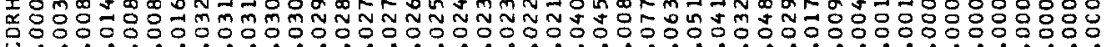

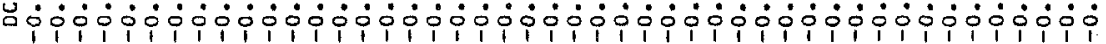

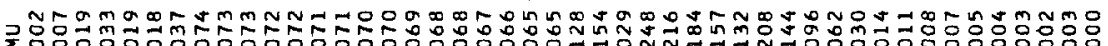

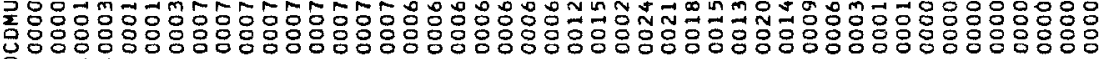

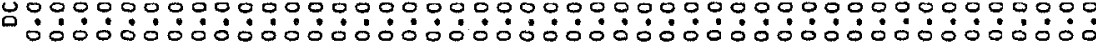
$\stackrel{\infty}{\infty}:$

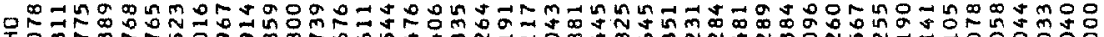

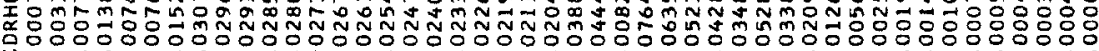

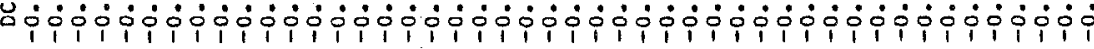

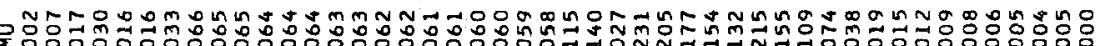

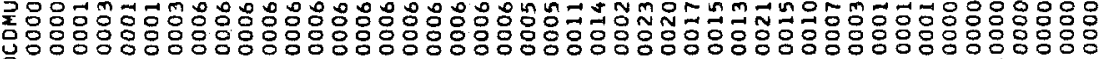

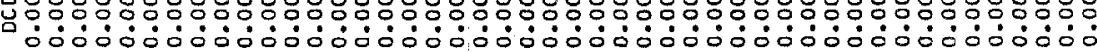

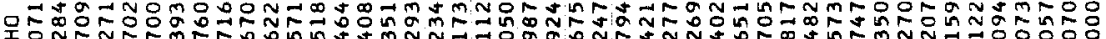

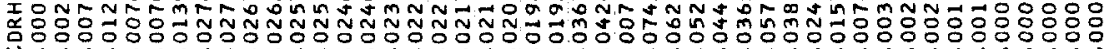

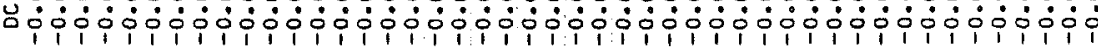
ว

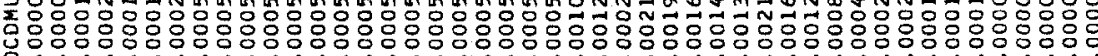

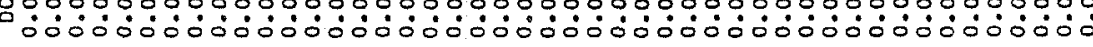
空员

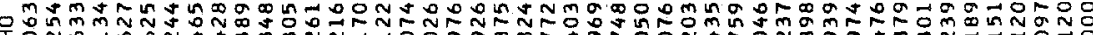

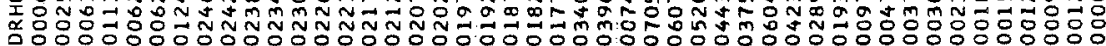

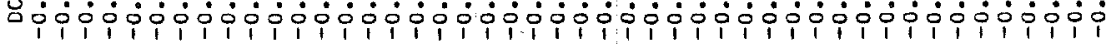

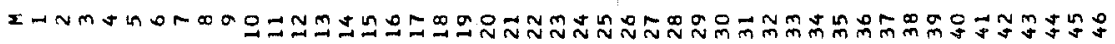




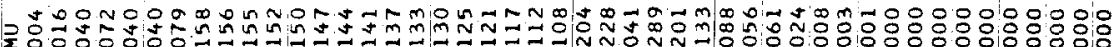

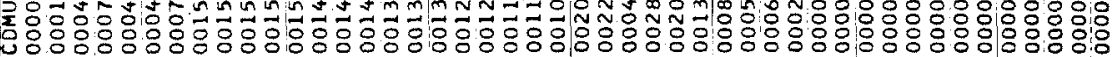

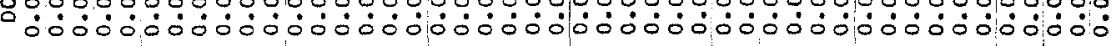

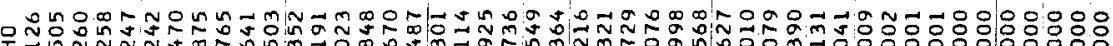

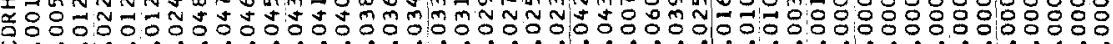

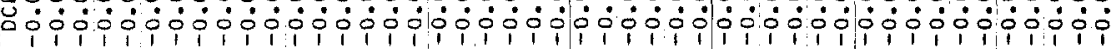

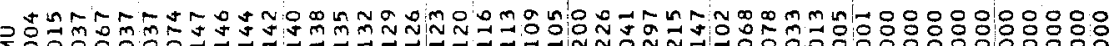

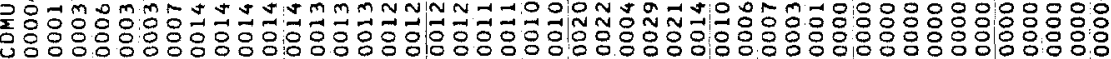

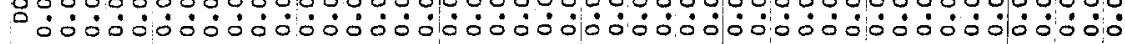

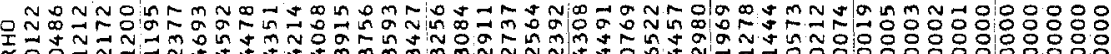

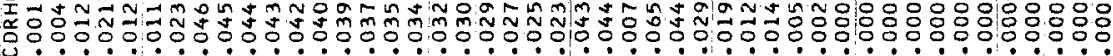

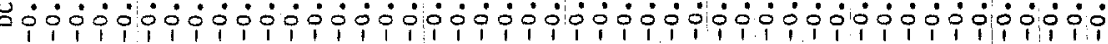

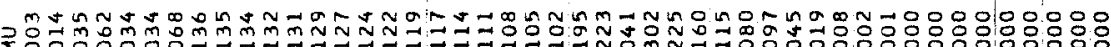

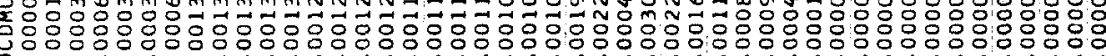

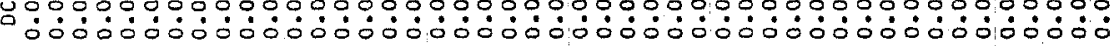

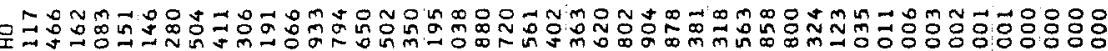

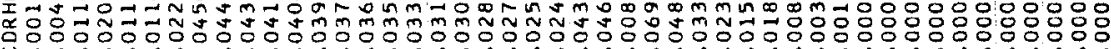

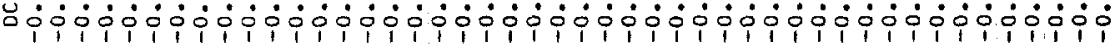

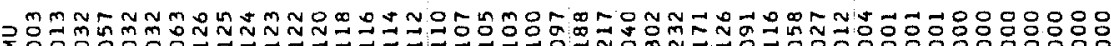

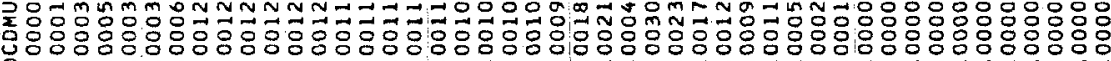
N 픈

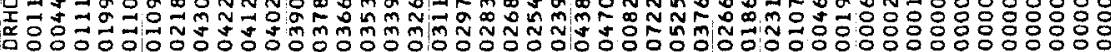

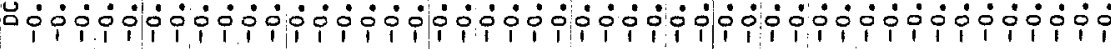
ํํำ

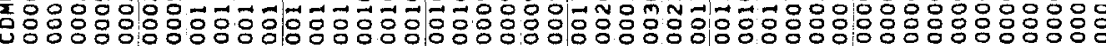

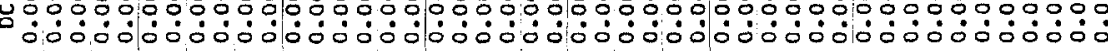

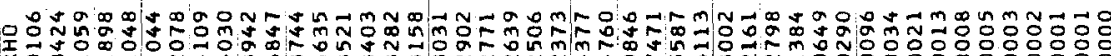

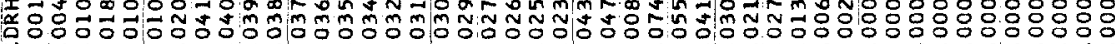

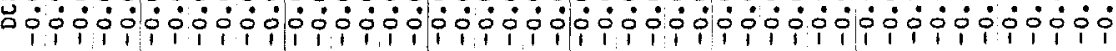

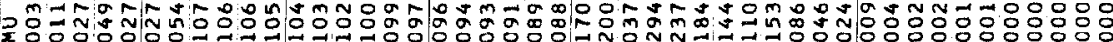

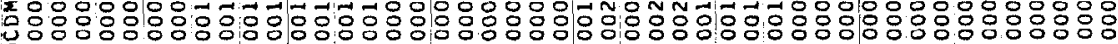

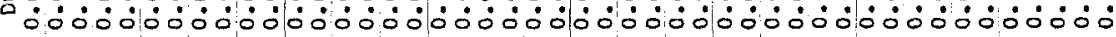

O.:

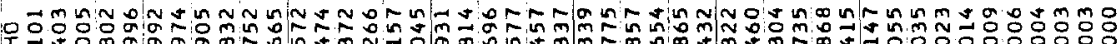

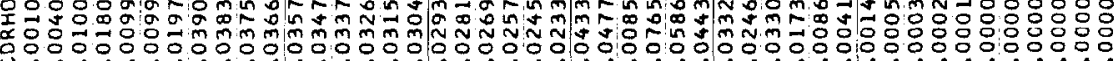

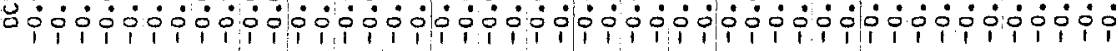

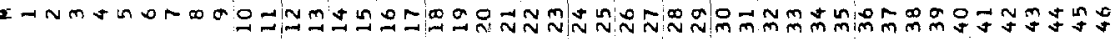




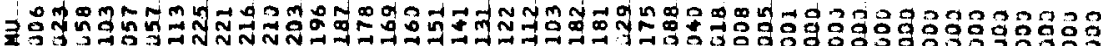

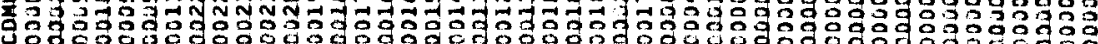

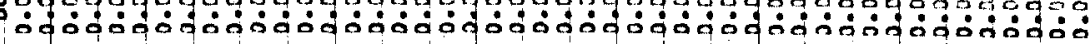

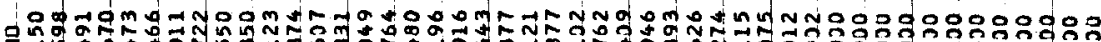

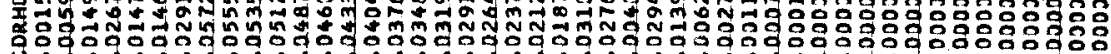

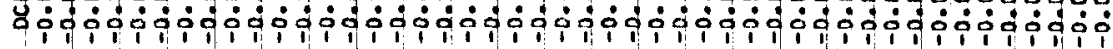

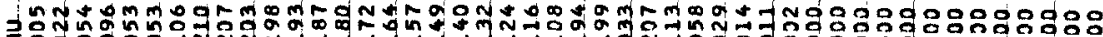

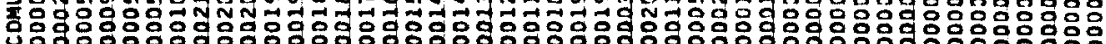

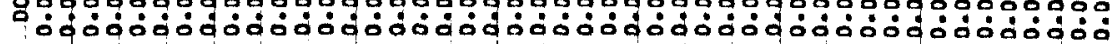

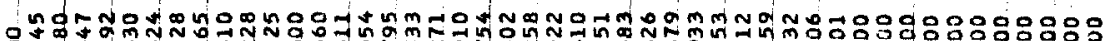

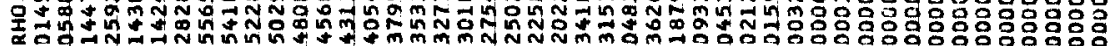

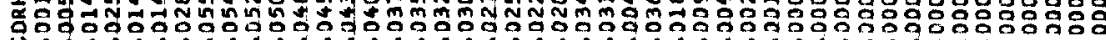

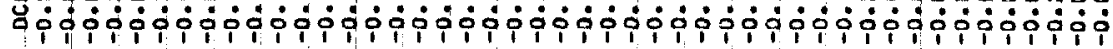

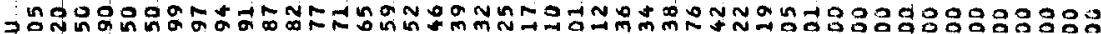

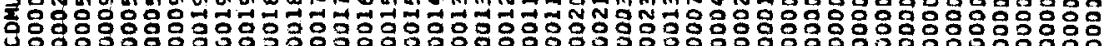

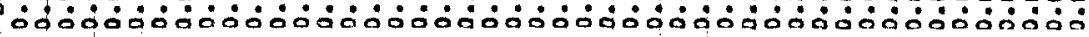

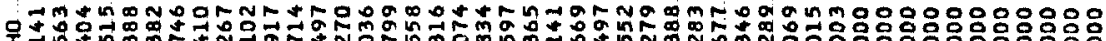

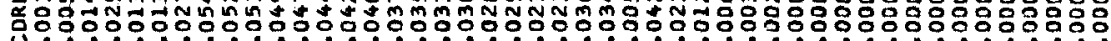

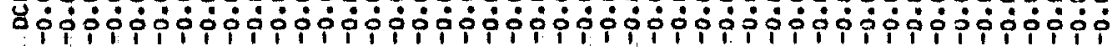

ว

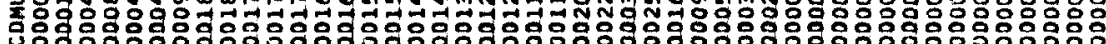

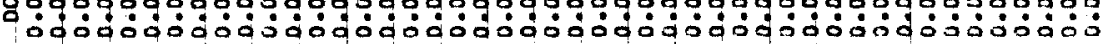

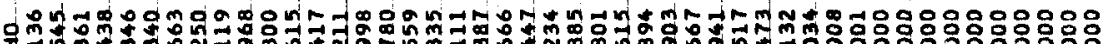

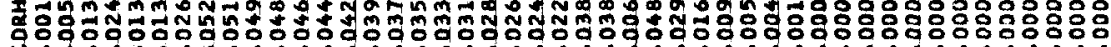

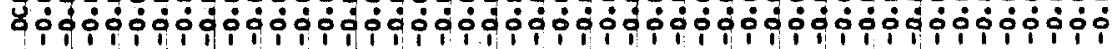

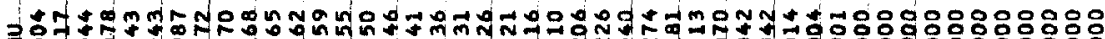

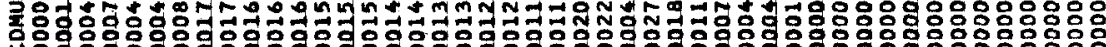

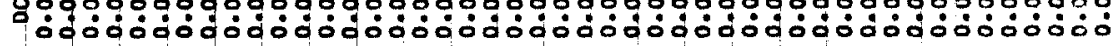

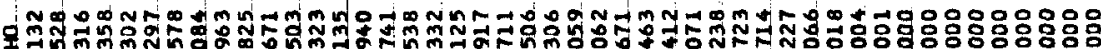

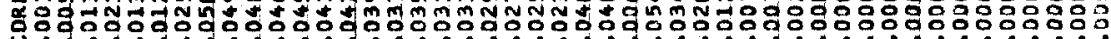

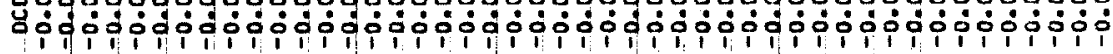

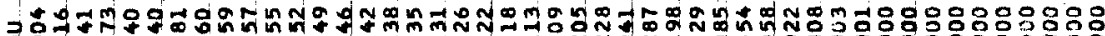

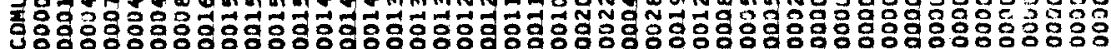

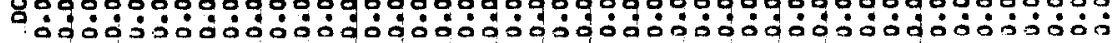

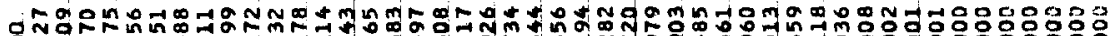




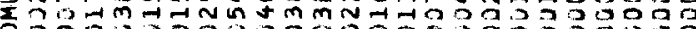

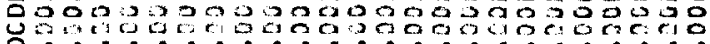

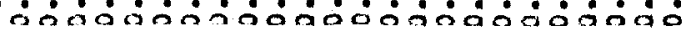

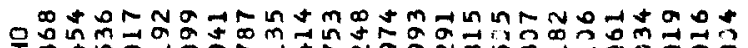

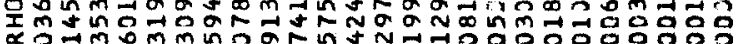

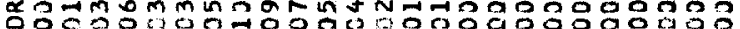

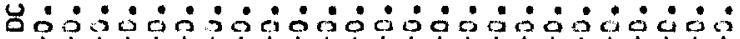

$\rightarrow m M a m N N a+t a n a m \rightarrow N N O \infty m \rightarrow-m O N N$ m

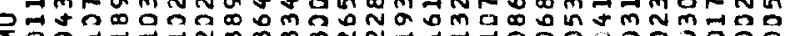

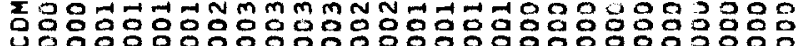

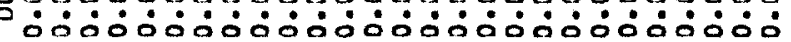

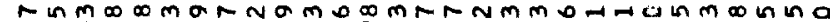

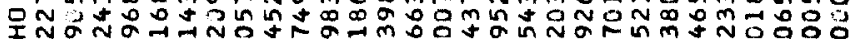

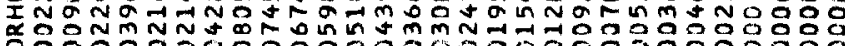

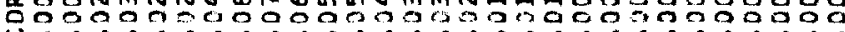

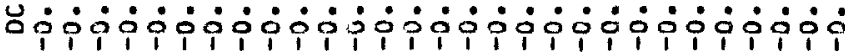

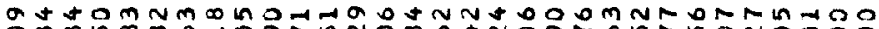

Jom 0 m

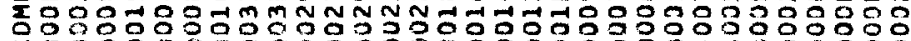

0
0
0
01

บ0003. 300000000000005003000000

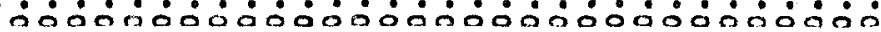

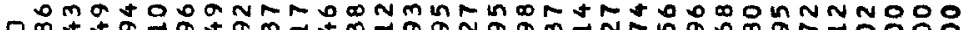

온휴

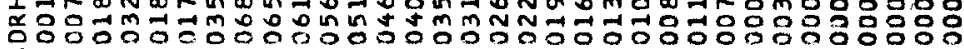

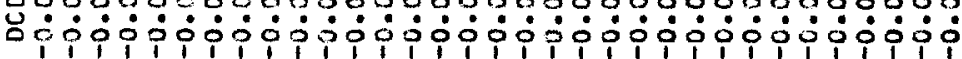

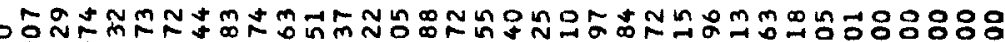

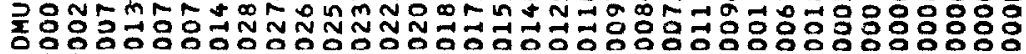

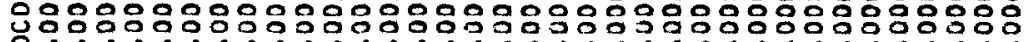

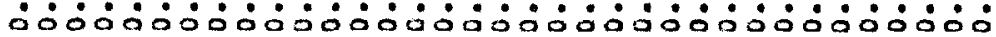

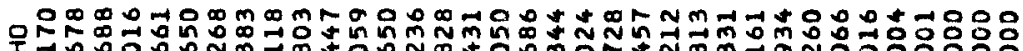

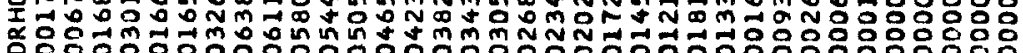

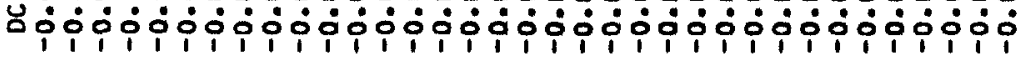

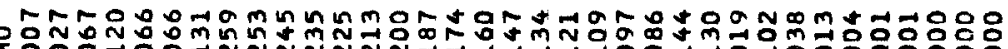

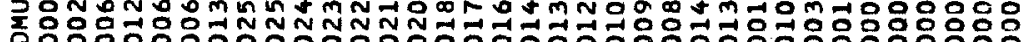

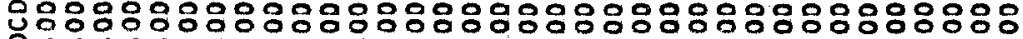

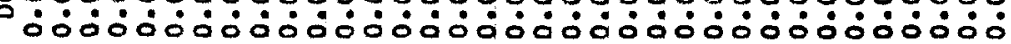

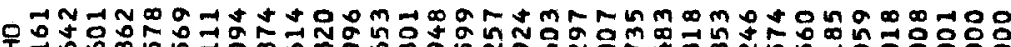

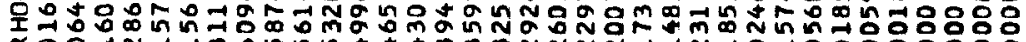

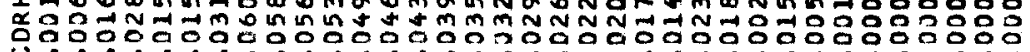

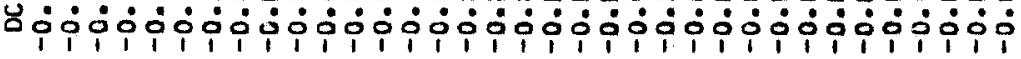

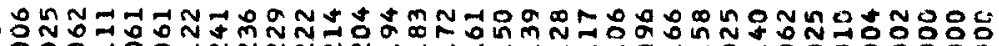

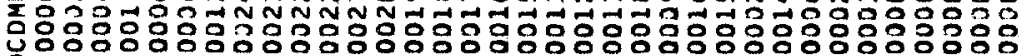

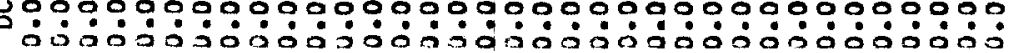

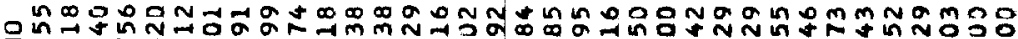

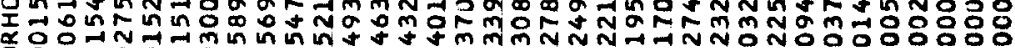

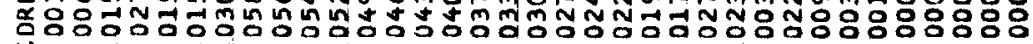

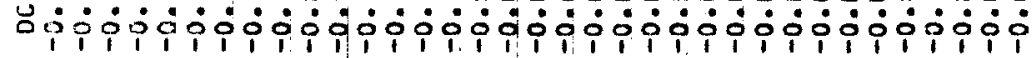

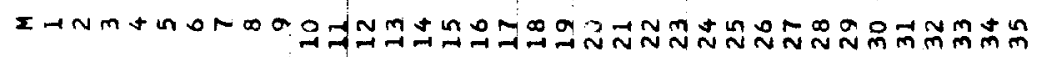




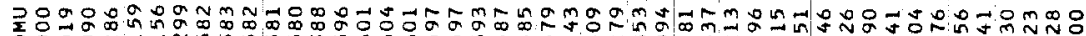

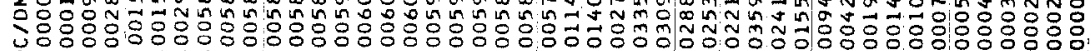

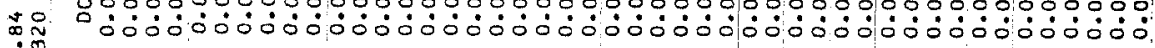

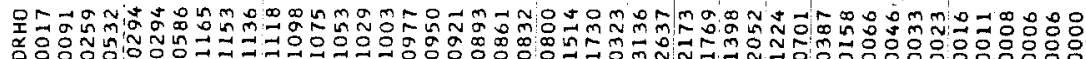

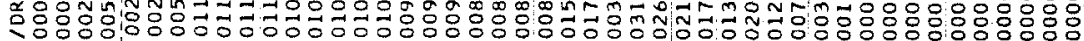

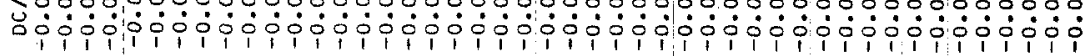

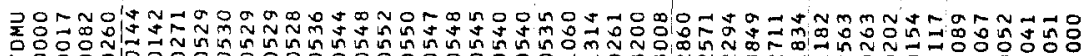

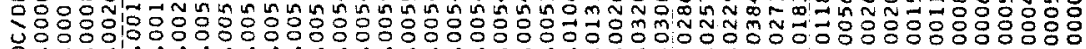

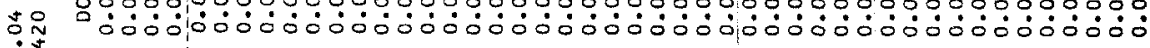

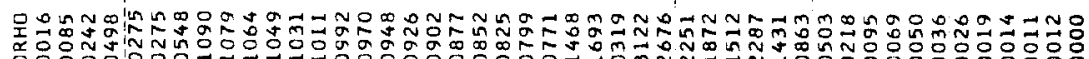

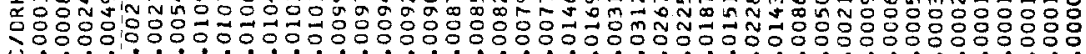

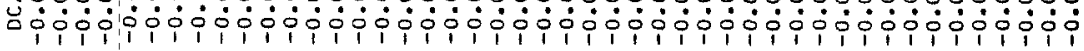

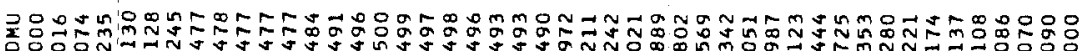

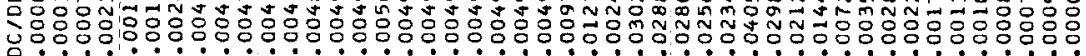

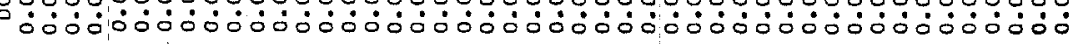

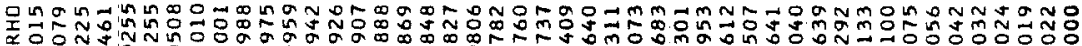

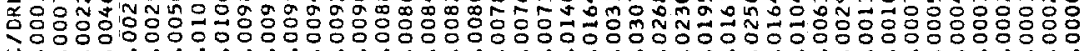

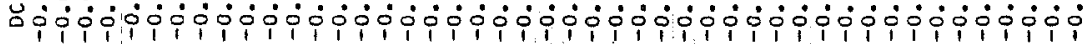

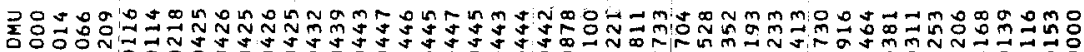

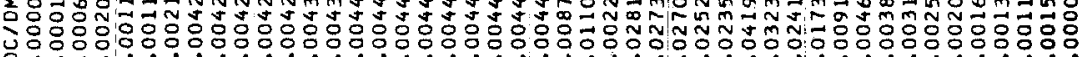

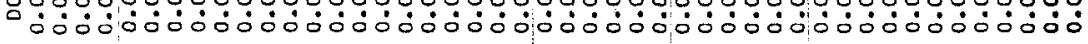

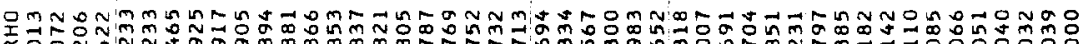

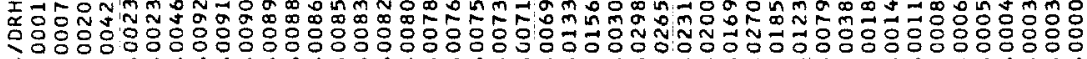

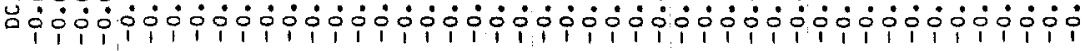

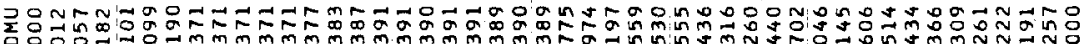

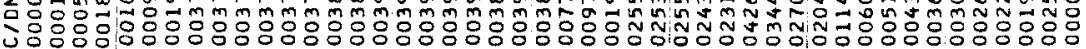

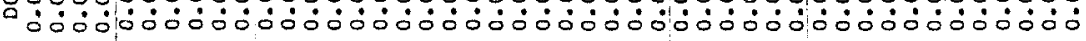

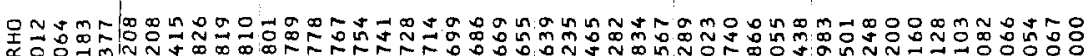

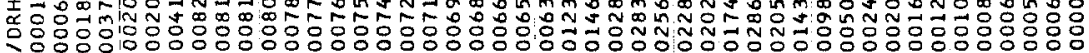

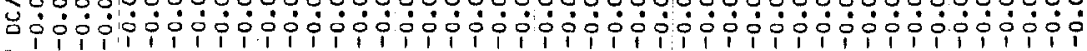

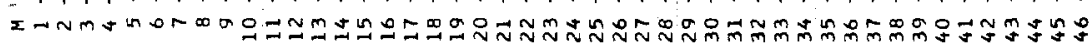




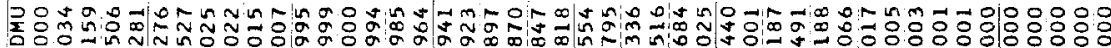

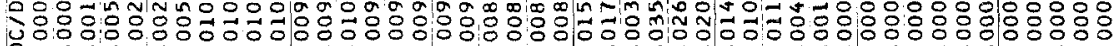

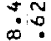

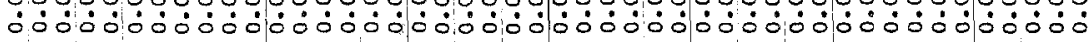
$\exists$

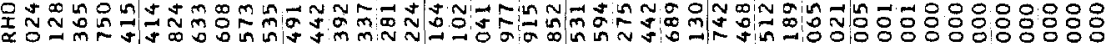

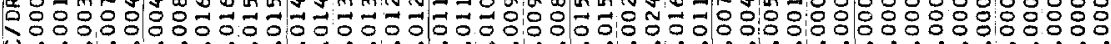

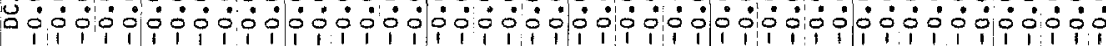

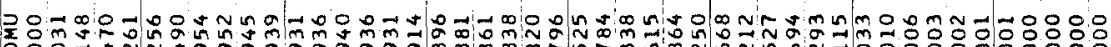

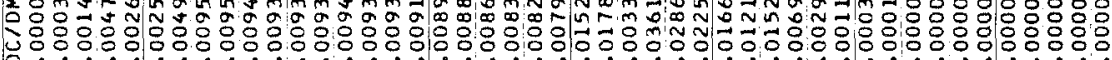

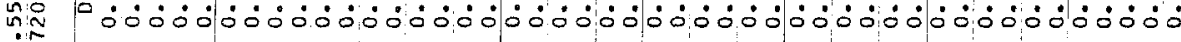
药in

渎 $N$ NN N

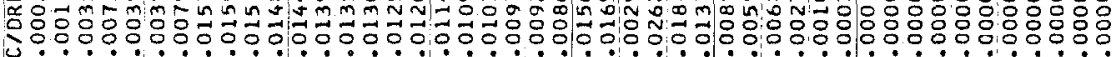

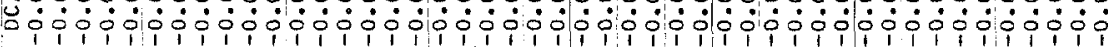

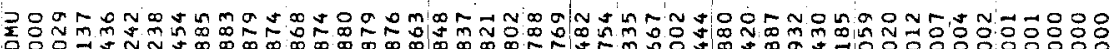

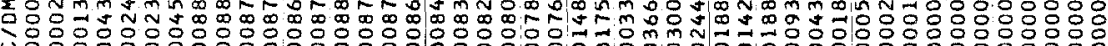
No

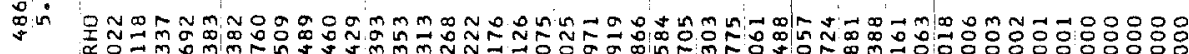

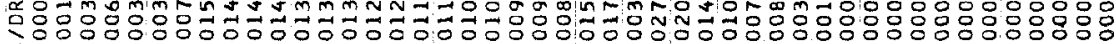

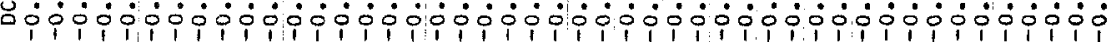

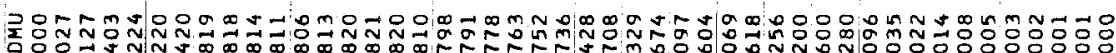

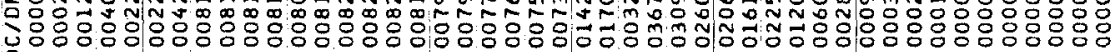
N

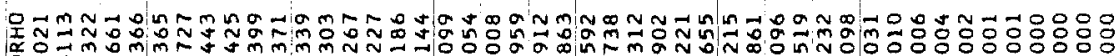

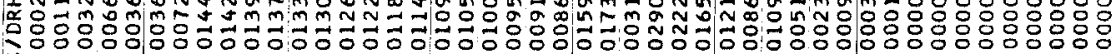

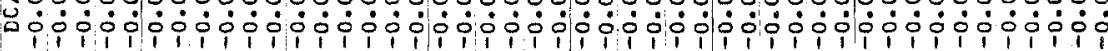

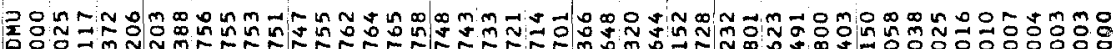

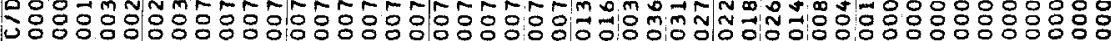

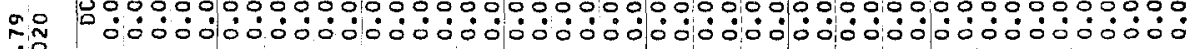
:ं:

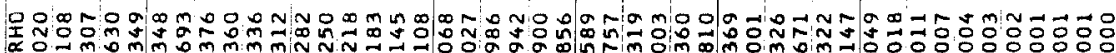

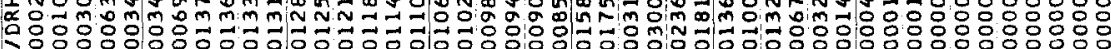

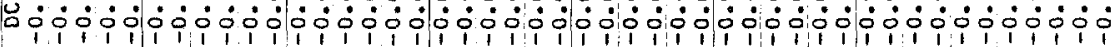

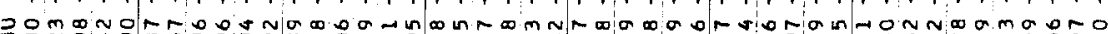

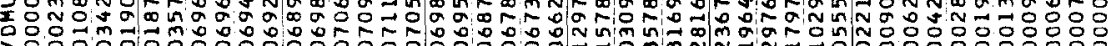

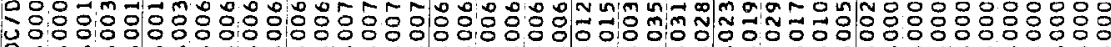

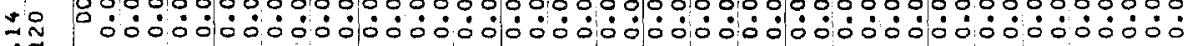

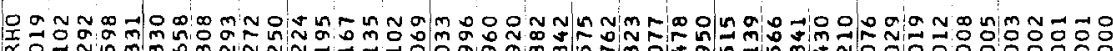

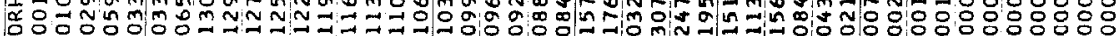

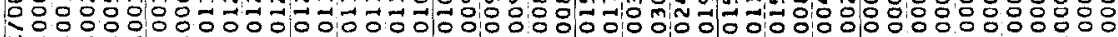

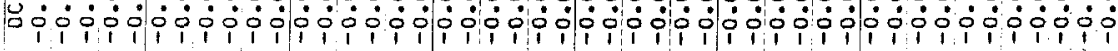

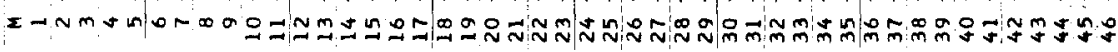




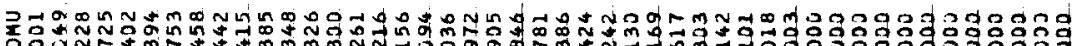

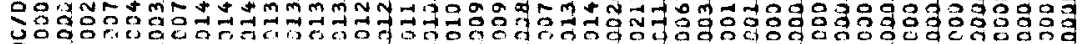

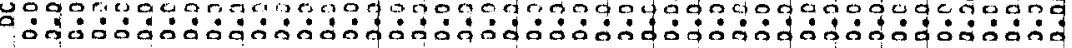

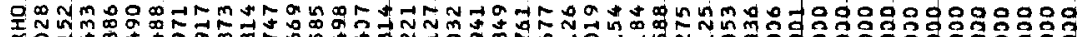

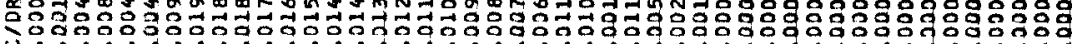

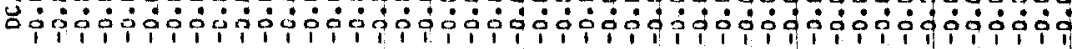
ว

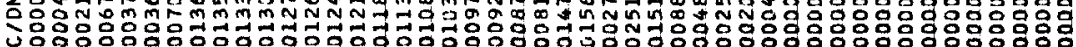

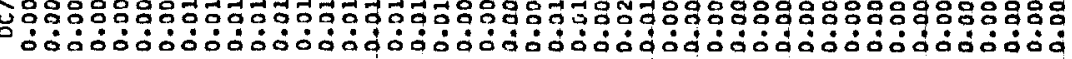

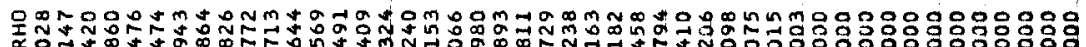

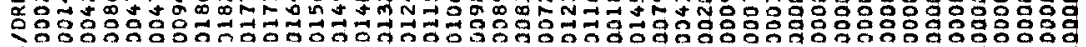

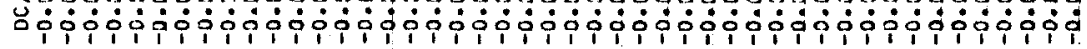

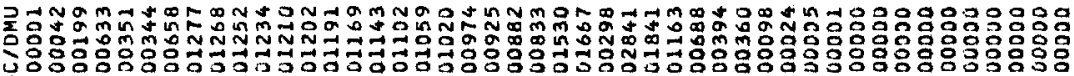

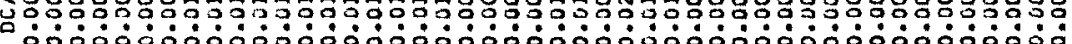

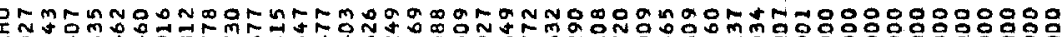

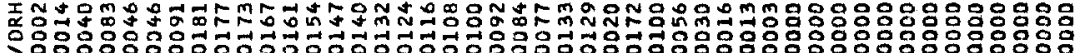

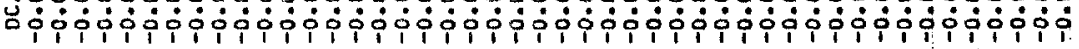

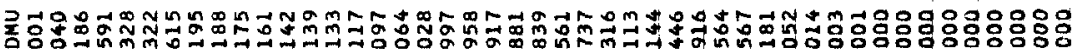

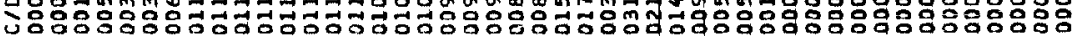

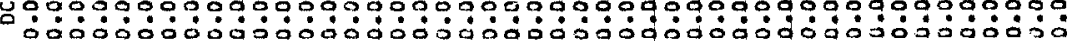

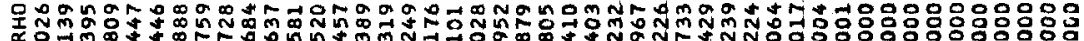

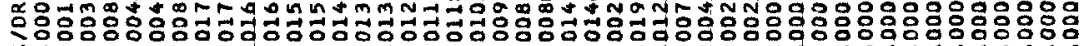

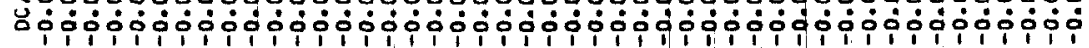

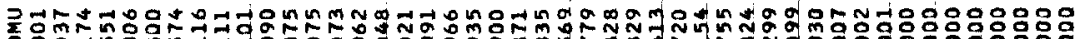

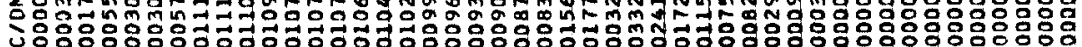

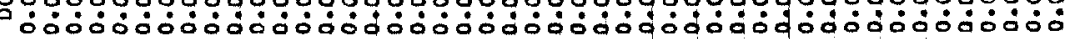

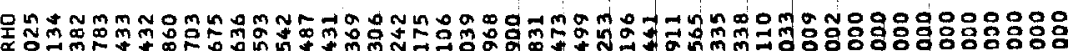

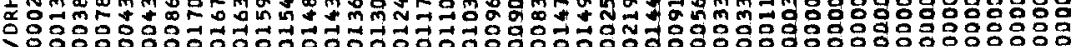

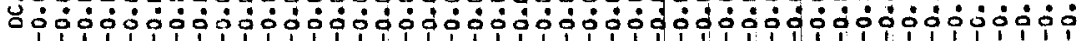

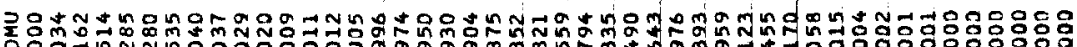

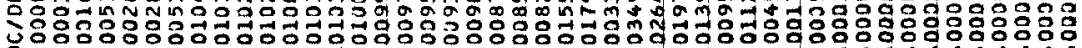

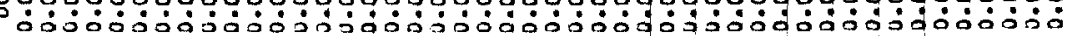

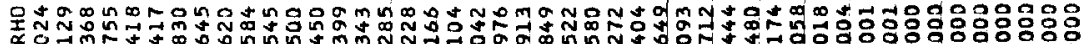

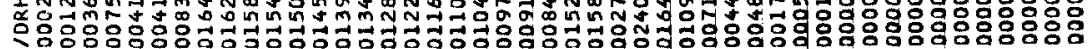

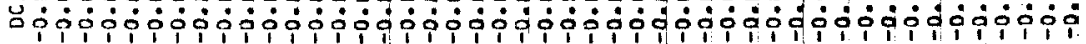
エ 


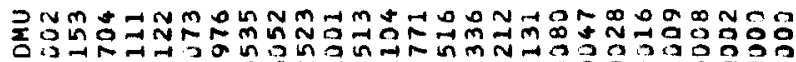

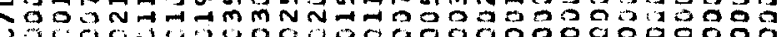
ó

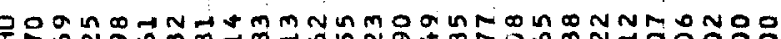

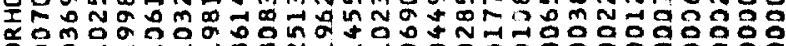

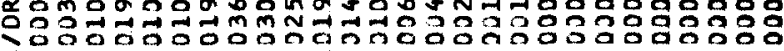

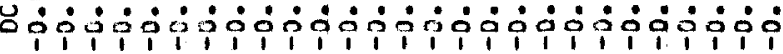

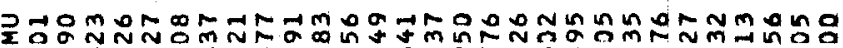

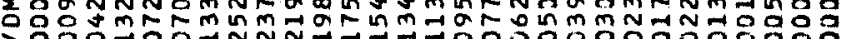

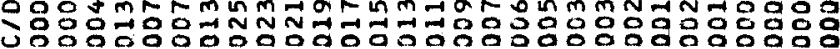

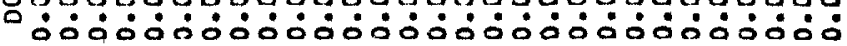

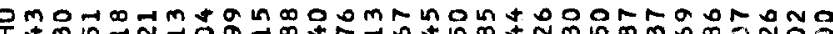

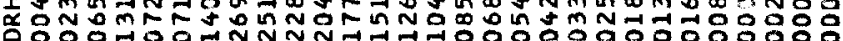

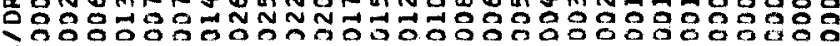

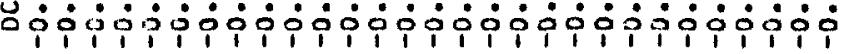

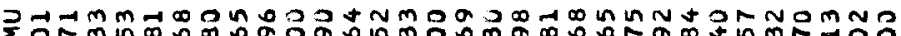

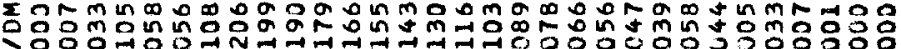

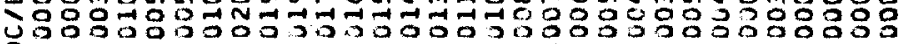

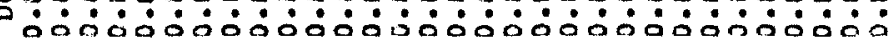

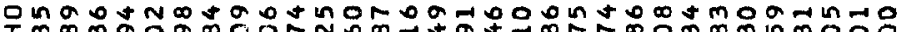

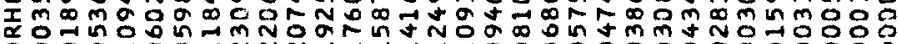

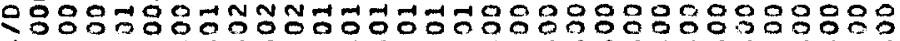

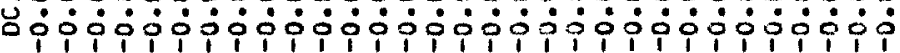

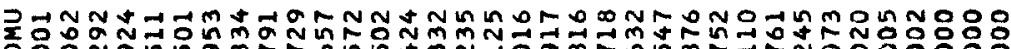
O유.

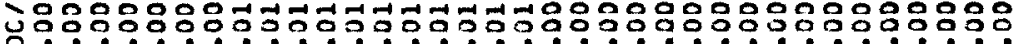

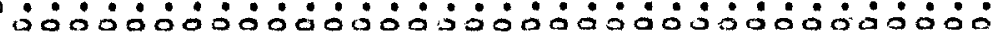

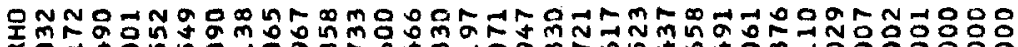

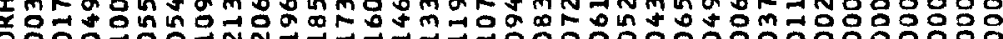

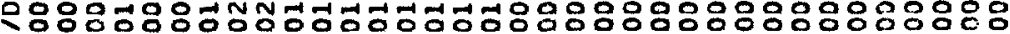

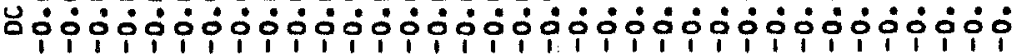

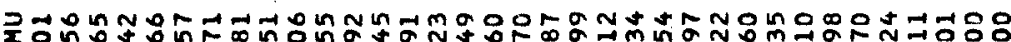

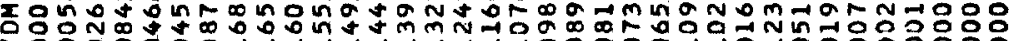

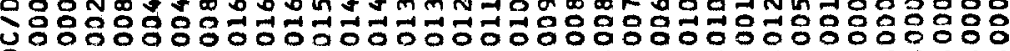

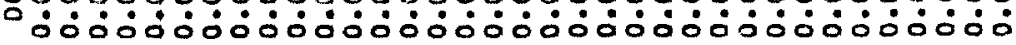

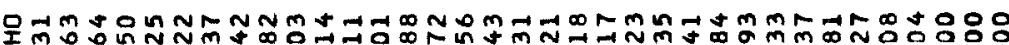

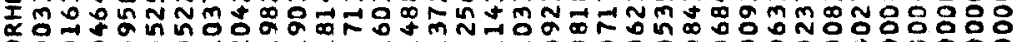

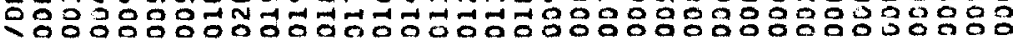

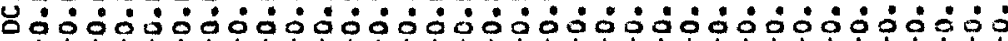

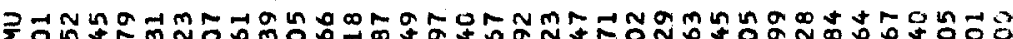

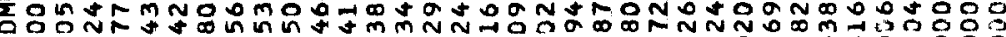

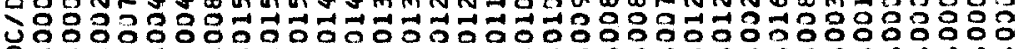

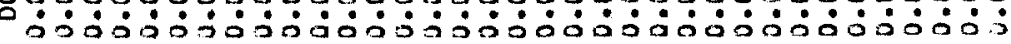

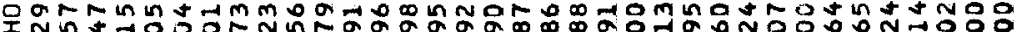

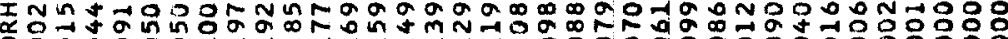

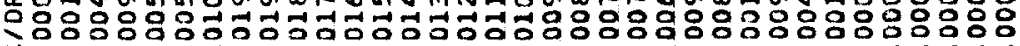

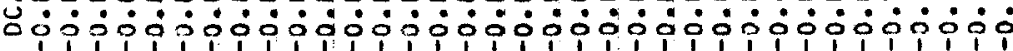

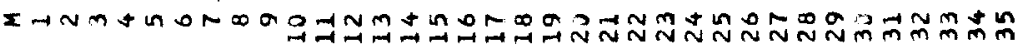




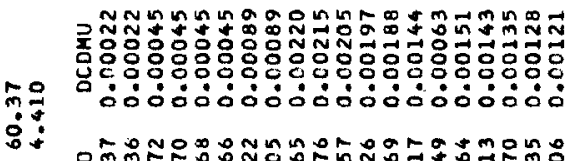

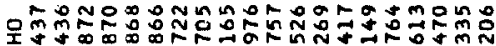

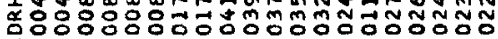

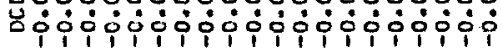

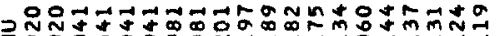

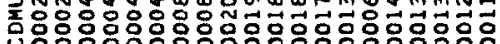
品

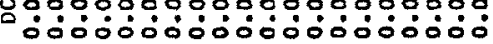

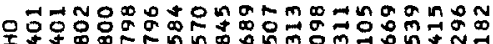

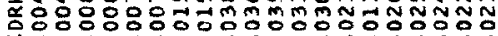

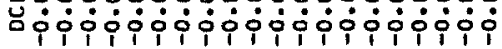
20

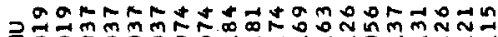

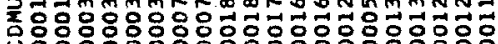

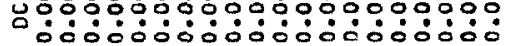
i:

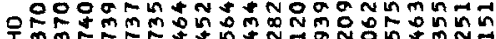

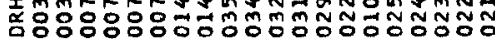

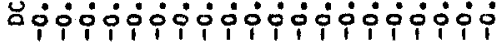

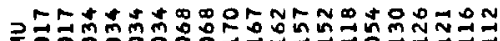

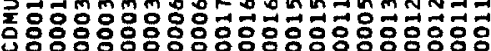

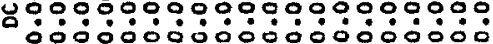
$\dot{m}:$

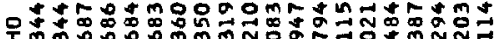

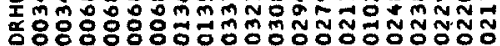

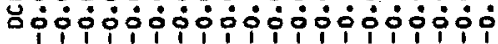

\section{mo}

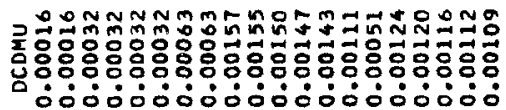

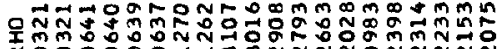


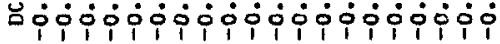

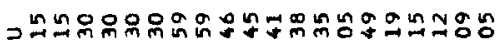

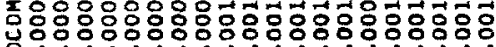

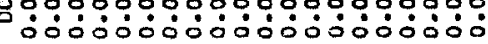

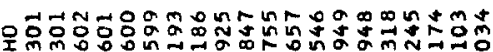

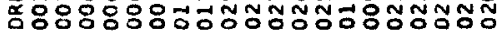

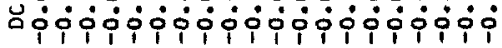

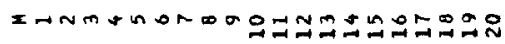

ปูn

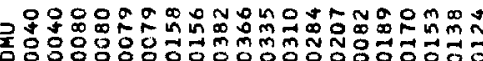

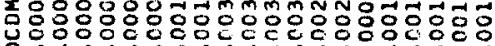
odódódódódiódódé

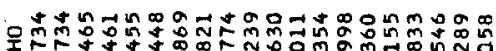

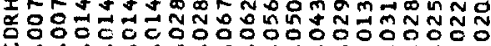

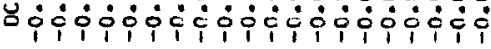

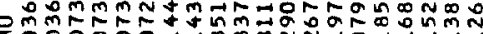

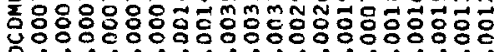

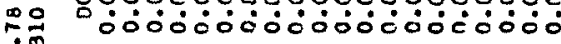

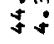

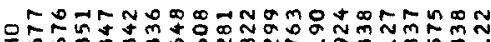

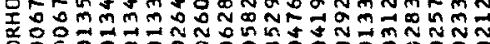

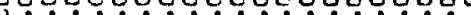

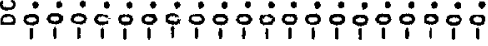

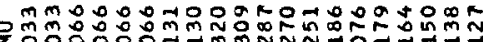

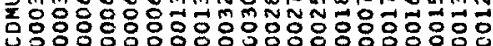

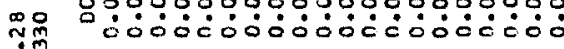

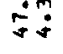

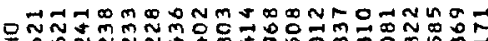

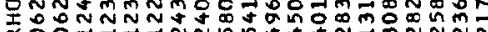

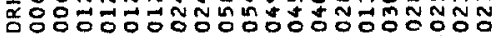
চipipipipipipipipioii

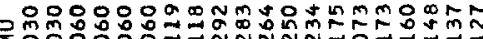

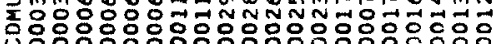

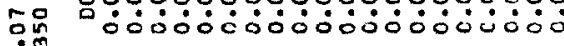

品:

웅ำ

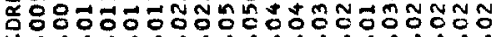

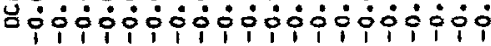

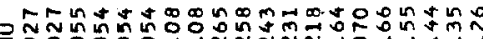

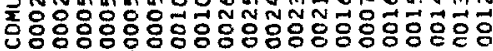

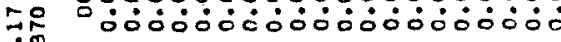
$\dot{m} \dot{m}$

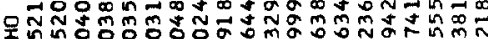
大

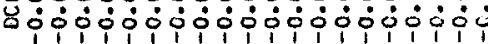

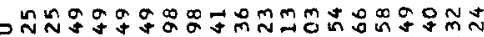
:음

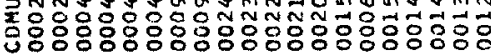

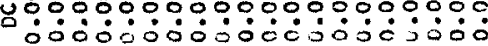

in

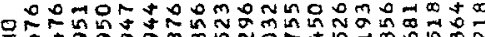

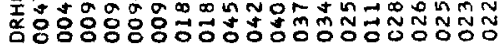

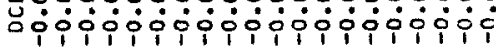

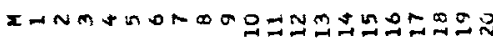




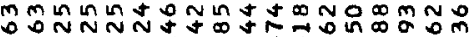

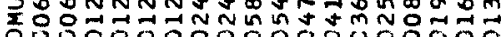
엉영영영ㅇㅇㅇ영영영영영

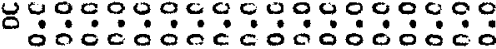
in:

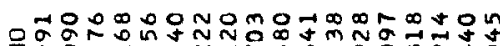

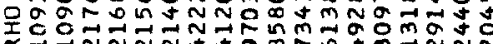

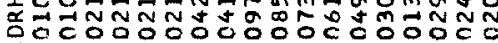

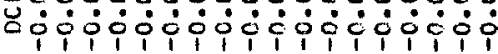

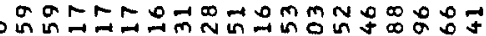
วิกำ

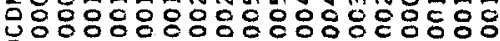

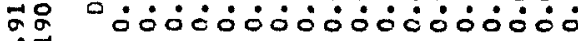
$\dot{m} \dot{m}$

om을

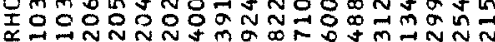

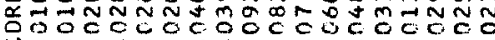

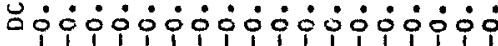

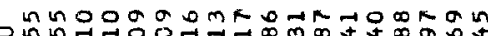

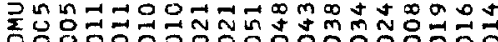

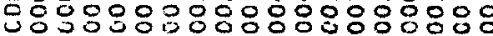

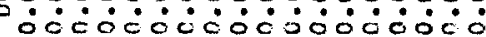
in:

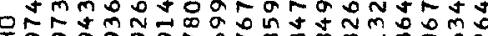

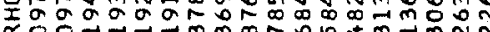

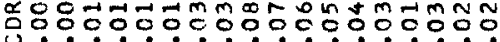

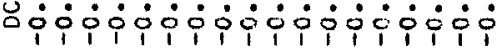

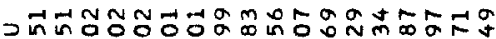
ㄷㅇ을

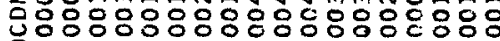

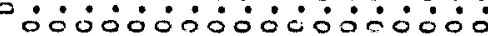

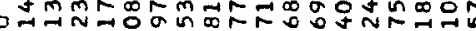

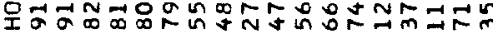

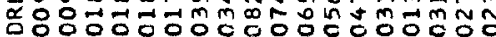

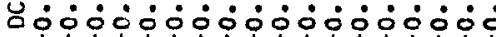

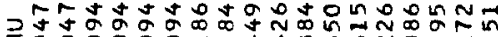

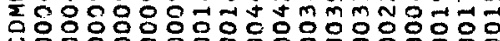

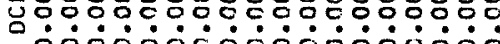

mmmnoOtom anomanoman

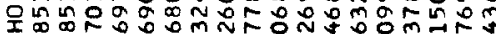

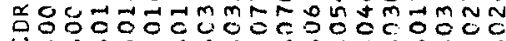

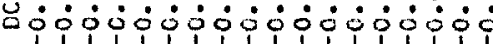

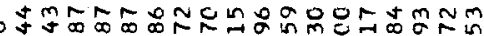
इ

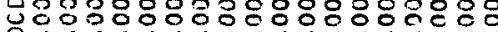
IR $\dot{0} \dot{0} \dot{0} \dot{0} \dot{0} \dot{0} \dot{0} \dot{0} \dot{0} \dot{0} \dot{0}$ 过

mmmonmngnomnmomn-

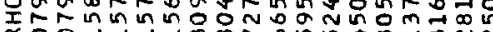

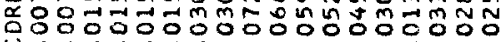

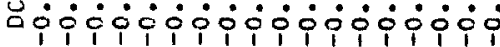

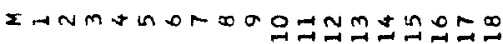

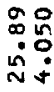

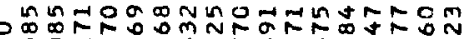

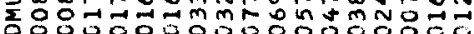

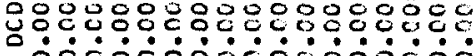

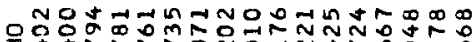
Thmominom:

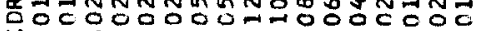

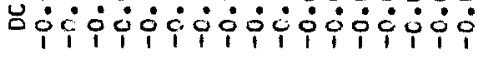

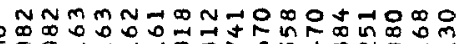

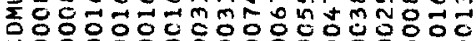

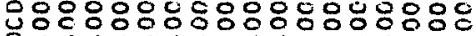

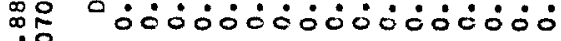
$\stackrel{\circ}{\circ}$

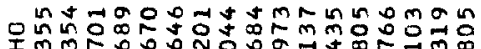

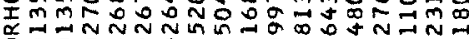

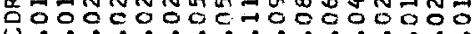
úióióióióiói món

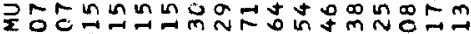

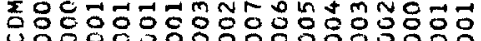

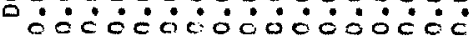

ongammatadomatom nn

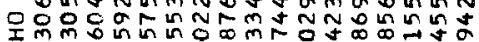

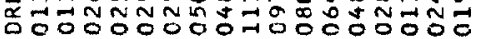

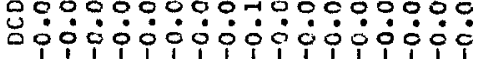

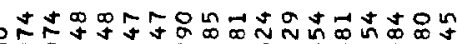

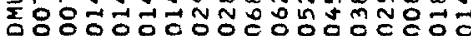

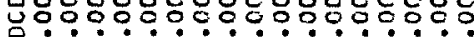
00000000000000000

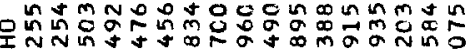

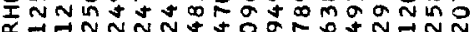

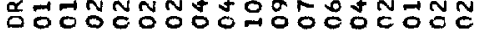

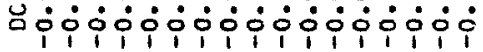

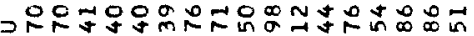

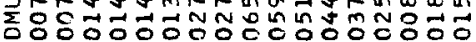

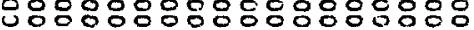
$\stackrel{N}{\because} \underset{m}{\stackrel{m}{*}}$

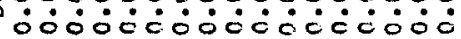

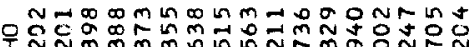

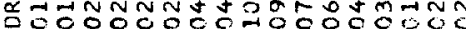

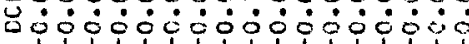

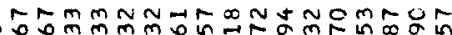
ว०ำmmm

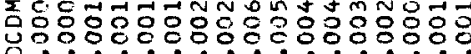
min mi

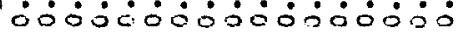

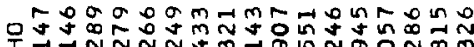

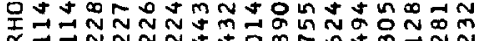
崩

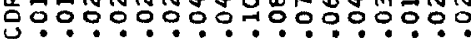
पóicóó

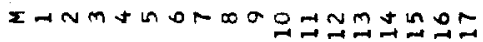


in 용 $\ddot{\sim} \dot{m}$

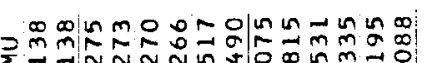

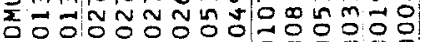

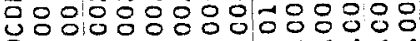

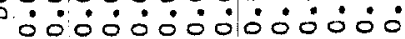

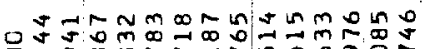

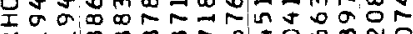

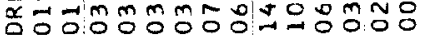

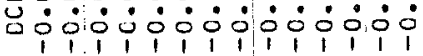

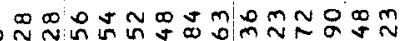

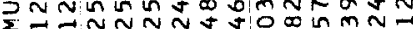

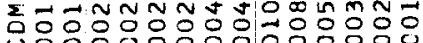

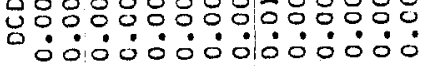

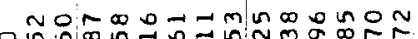

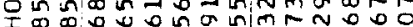

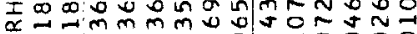

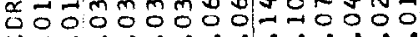

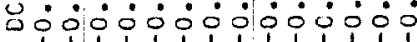

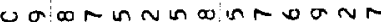

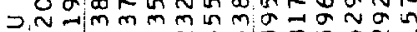

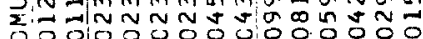

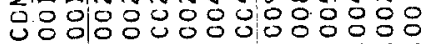

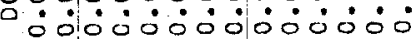

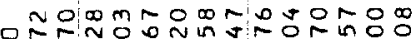

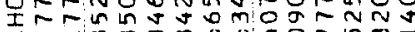

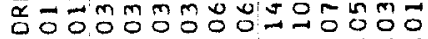

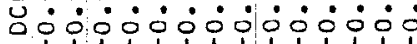

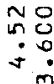

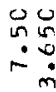

ป约 50.

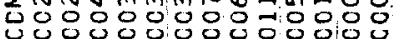

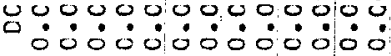

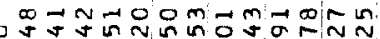

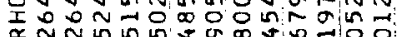

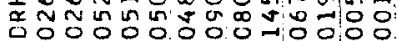

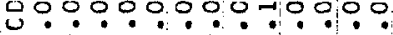

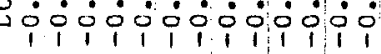
O

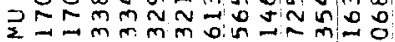

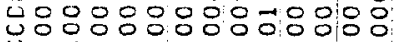
ó $\dot{0} \dot{0} \dot{0} \dot{0} 0 \dot{0} 0 \dot{0}$

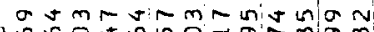

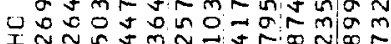

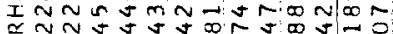

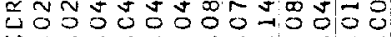

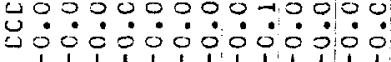
1

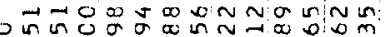
s

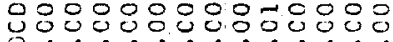

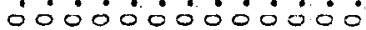

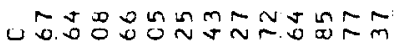

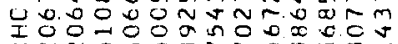

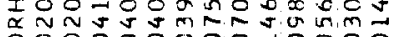

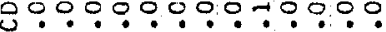

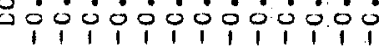
2-nmonoroog 0 an

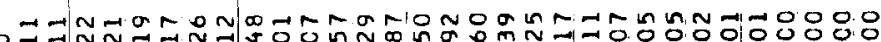
วีష

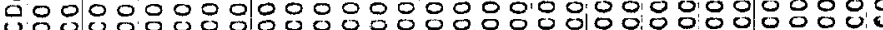

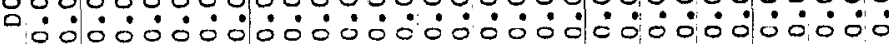

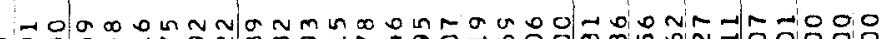

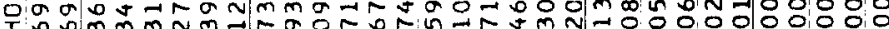
1 o o00000000 Ú mmñm

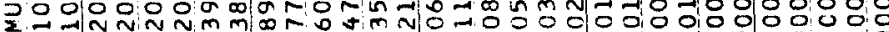

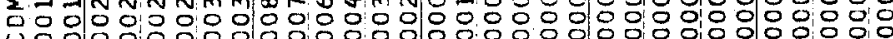

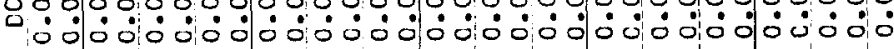

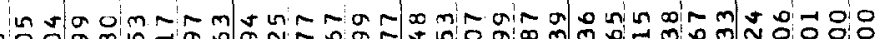

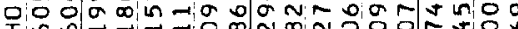
至

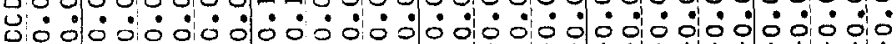

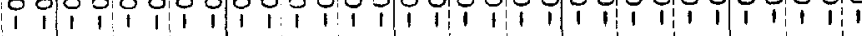

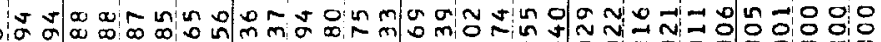

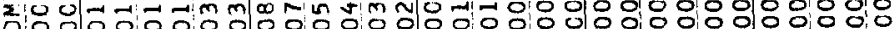

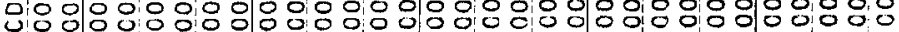
ó a amonamor

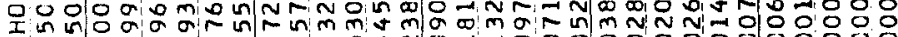

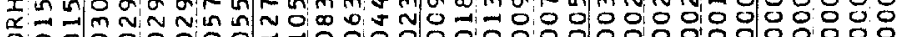

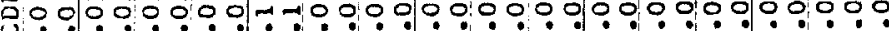

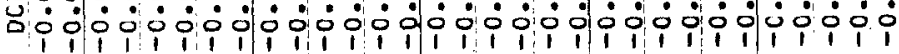

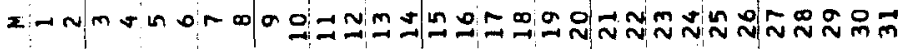




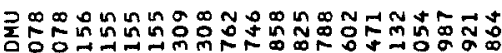

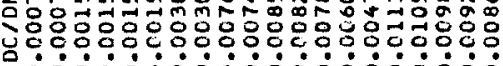

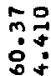

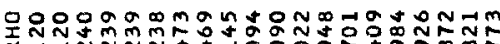

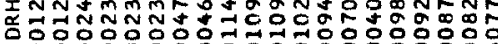
-óióiói

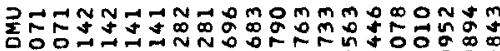

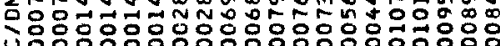

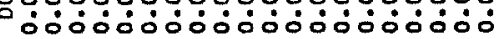

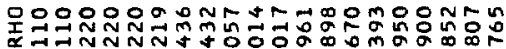

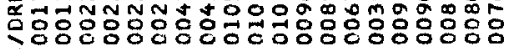
L

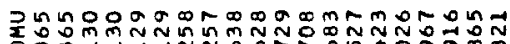

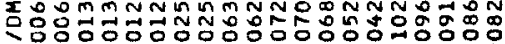

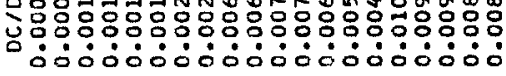

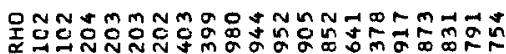

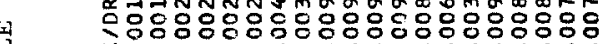

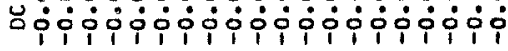

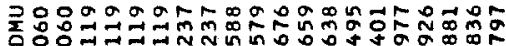

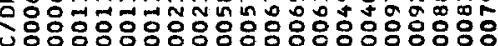

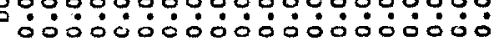

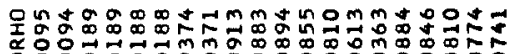

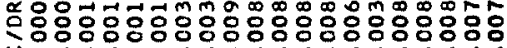

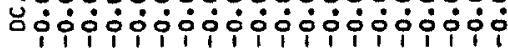

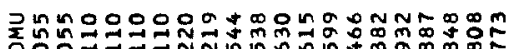

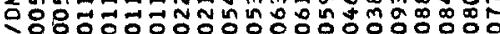

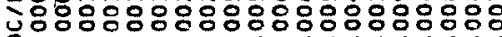

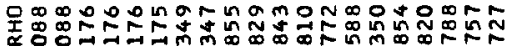

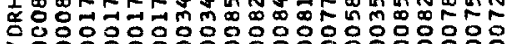

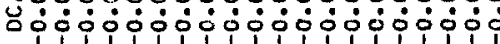

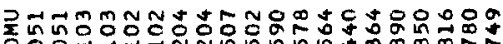

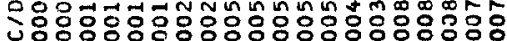

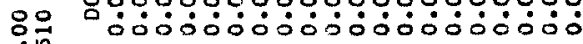

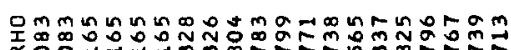

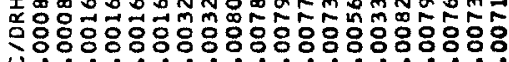
0 0000000000000000000 $x \rightarrow N m+m 0$ - in은

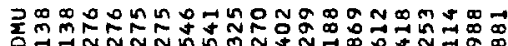

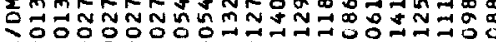

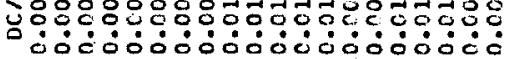
$\stackrel{4}{4}$

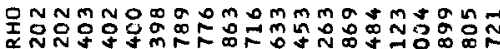

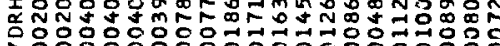

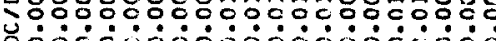
保审i

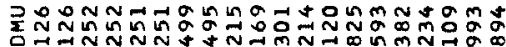

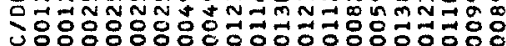
 id

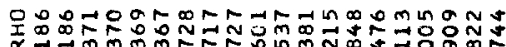

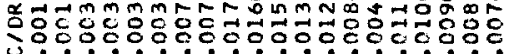

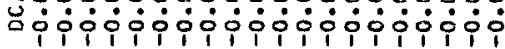

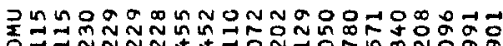

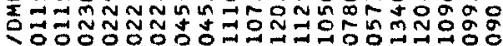

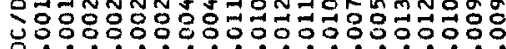

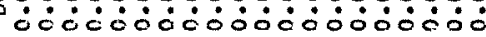

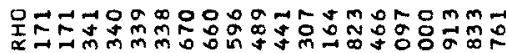

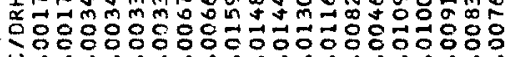

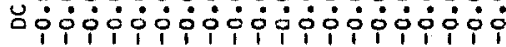

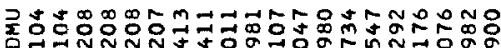

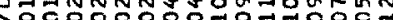

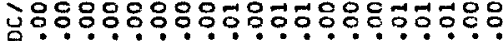

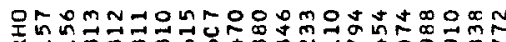

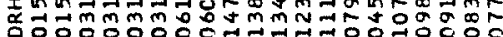

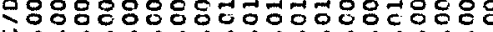

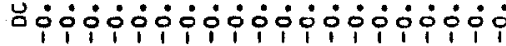

ว

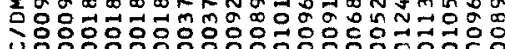

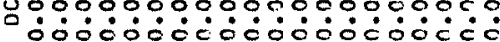
뭉 nis

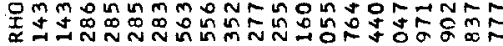

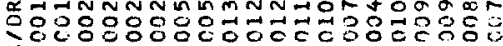

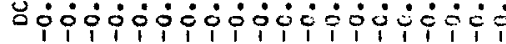

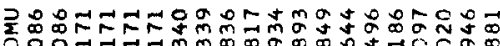

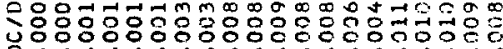
óं0்

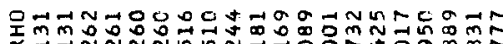

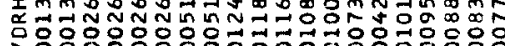

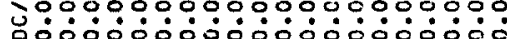




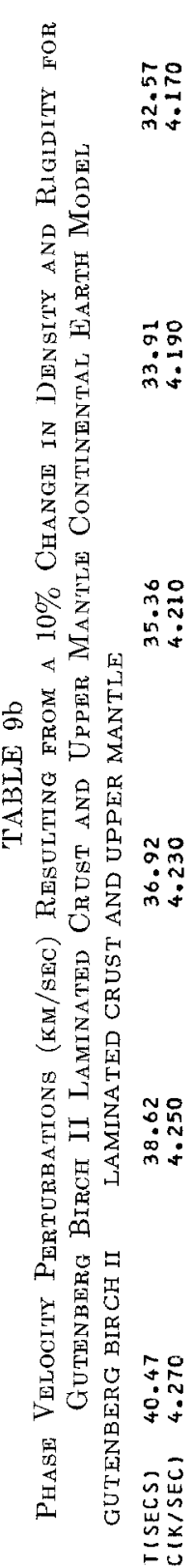

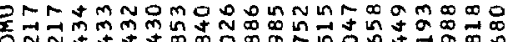

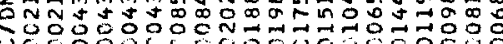

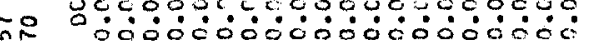
in:

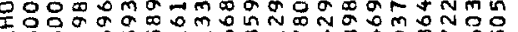

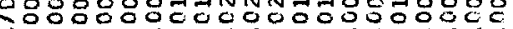

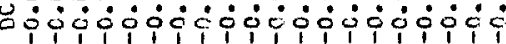

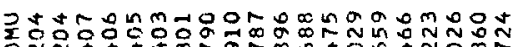

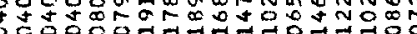

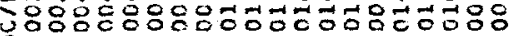

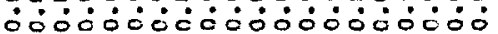

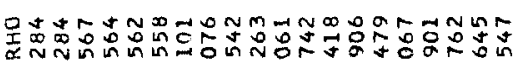

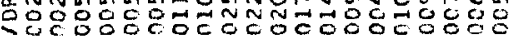

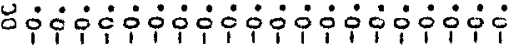

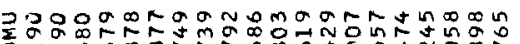

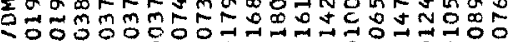

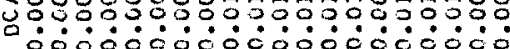

$\ddot{m}$

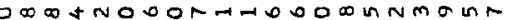

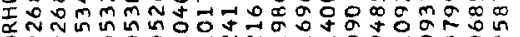

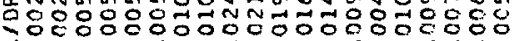
Lipipipipioipipioíip

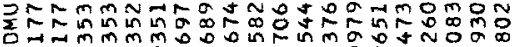

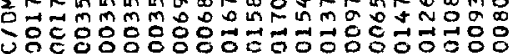

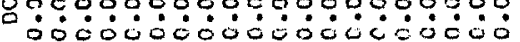

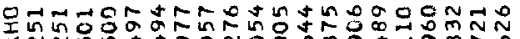

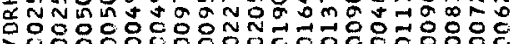

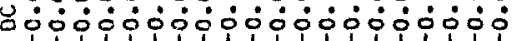

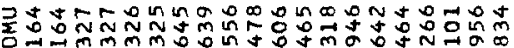

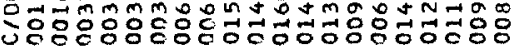

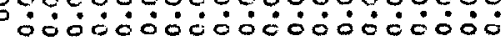

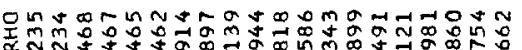

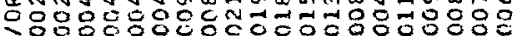

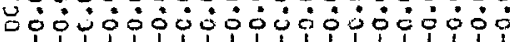

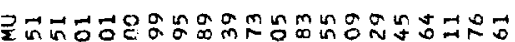

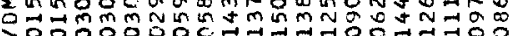

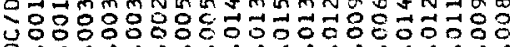

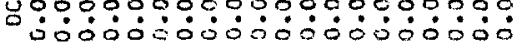

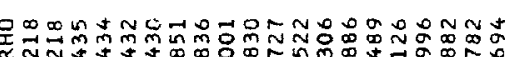
ลี่ง

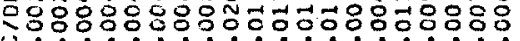
upipiciiiiiiiiii

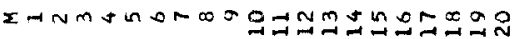

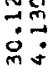

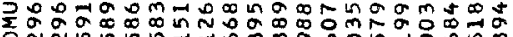
NNin nun

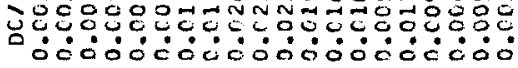

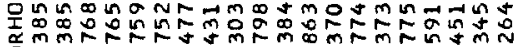

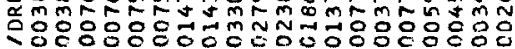

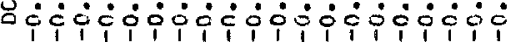

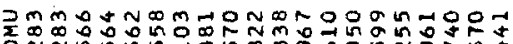

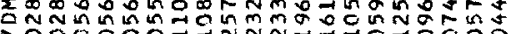

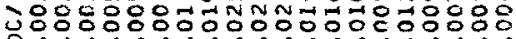

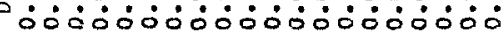

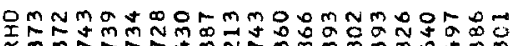

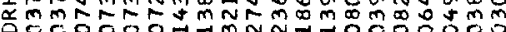

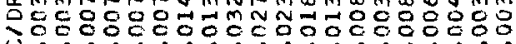

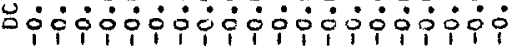

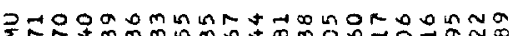

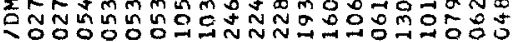

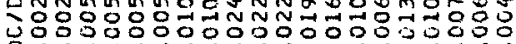

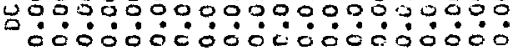

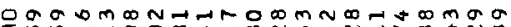

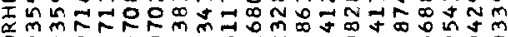

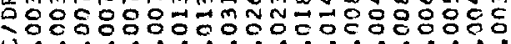
upoipoipopipipipipipii

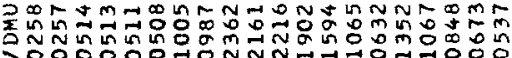

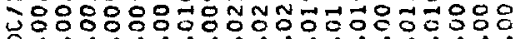

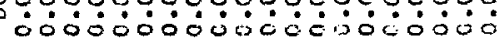

우순

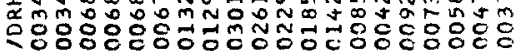
ăipipipiopipipipipi

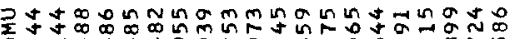

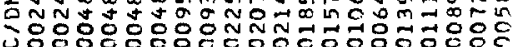

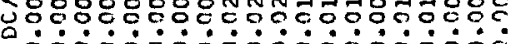

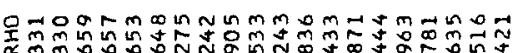

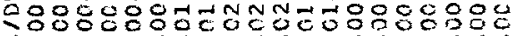

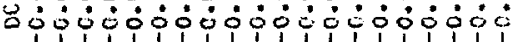

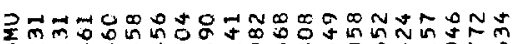

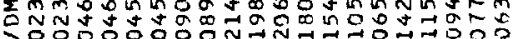

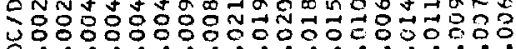

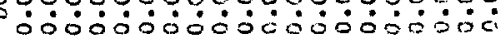

Qnn

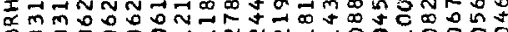

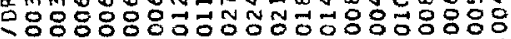
-ipipipipipipipipipio

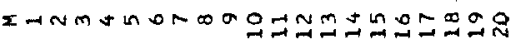




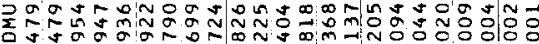

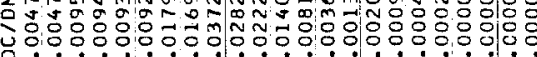

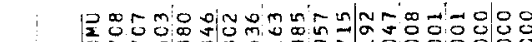

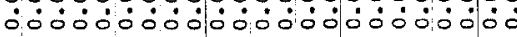

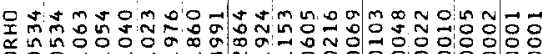

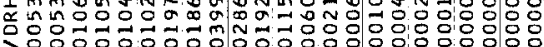

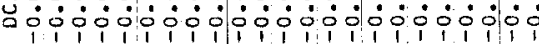

ज:

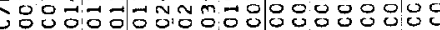

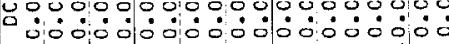

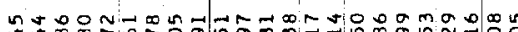

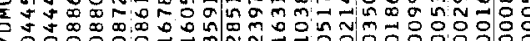

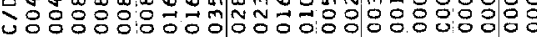

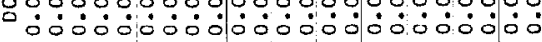

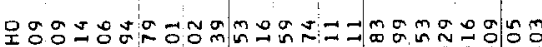

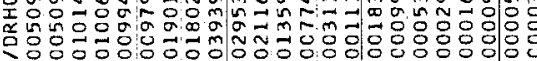

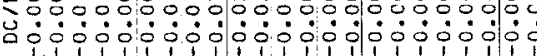

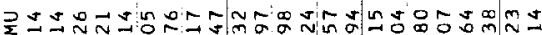

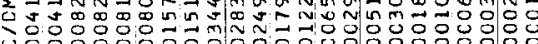

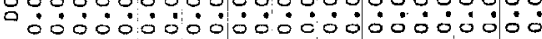

呈

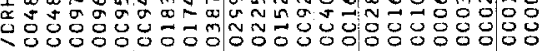

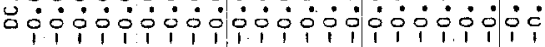

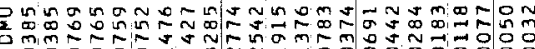

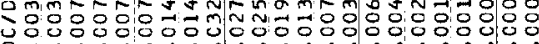

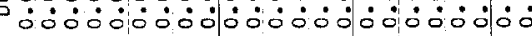

$\dot{m}$ INNG a n u ó jó ó

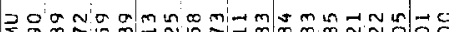

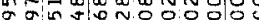

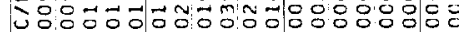

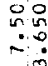

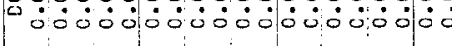

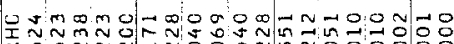
萑

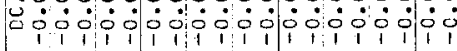
₹nn

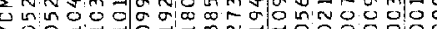
요유 $\leadsto$ 品 $\rightarrow m$

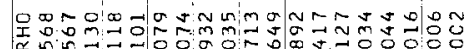

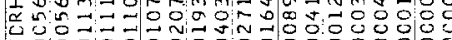
च̄u wi

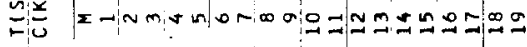

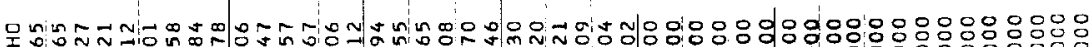
T. ¿́ó

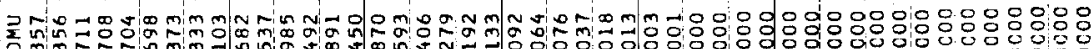

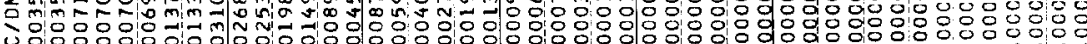
ó

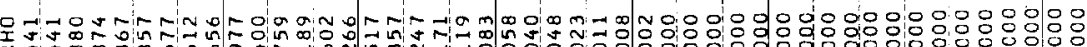

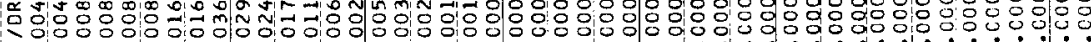
U ó ว

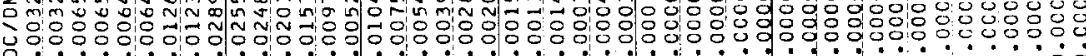
ó

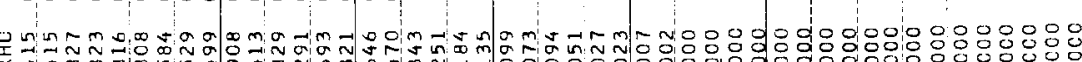

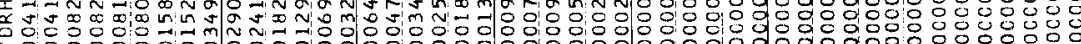

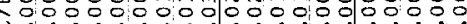
过

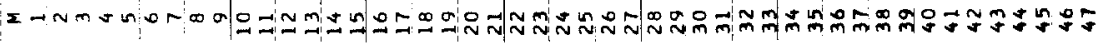


particular problem by modifying the trial structures and comparing the "predicted" results with those computed or published elsewhere. All published theoretical Love wave dispersion curves should be reproducible from the tables given here.

TABLE 10a

Variational Partial Demivatives for CIT13F Laminated Crust and Upper Mantle Oceanic Earth Modet

CITI3F OCEANIC LAMINATED CRUST AND UPPER MANTLE

\begin{tabular}{|c|c|c|c|c|c|c|c|c|c|c|c|c|}
\hline \multicolumn{3}{|c|}{$\begin{array}{l}\text { PSEUDO SPHERICAL } \\
\text { TISECSI } 119.11 \\
\text { C(K/SECI } 4.700\end{array}$} & & & \multicolumn{2}{|c|}{$\begin{array}{l}78.15 \\
4.600\end{array}$} & \multicolumn{2}{|c|}{$\begin{array}{l}57.53 \\
4.550\end{array}$} & \multicolumn{2}{|c|}{$\begin{array}{l}38.61 \\
4.500\end{array}$} & \multicolumn{2}{|c|}{$\begin{array}{l}23.42 \\
4.450\end{array}$} \\
\hline $\begin{array}{r}M \\
1 \\
2 \\
3 \\
4 \\
5 \\
6 \\
7 \\
8 \\
9 \\
10 \\
11\end{array}$ & $\begin{array}{l}\text { DCDRHO } \\
-0.00037 \\
-0.00074 \\
-0.00074 \\
-0.00186 \\
-0.00186 \\
-0.00371 \\
-0.00371 \\
-0.00740 \\
-0.00738 \\
-0.00736 \\
-0.00733 \\
-0.00731 \\
-0.00729 \\
-0.00364 \\
-0.01812 \\
-0.01798 \\
-0.01783 \\
-0.01767 \\
-0.01749 \\
-0.01731 \\
-0.01711 \\
-0.01689 \\
-0.03314 \\
-0.03216 \\
-0.03111 \\
-0.02998 \\
-0.05650\end{array}$ & $\begin{array}{l}\text { DCDMU } \\
0.00002 \\
0.00003 \\
0.00003 \\
0.00008 \\
0.00008 \\
0.00017 \\
0.00017 \\
0.00034 \\
0.00034 \\
0.00533 \\
0.00733 \\
0.001133 \\
0.00033 \\
0.00017 \\
0.00583 \\
0.00082 \\
0.00582 \\
0.00081 \\
0.00081 \\
0.00080 \\
0.00079 \\
0.00578 \\
0.00154 \\
0.00151 \\
0.00147 \\
0.00142 \\
0.00271\end{array}$ & $\begin{array}{l}\text { DCDRHO } \\
-0.00040 \\
-0.00081 \\
-0.00080 \\
-0.00291 \\
-0.00201 \\
-0.00401 \\
-0.0 .0401 \\
-0.00799 \\
-0.00796 \\
-0.00793 \\
-0.00790 \\
-0.00787 \\
-0.00785 \\
-0.00391 \\
-0.01947 \\
-0.01929 \\
-0.01910 \\
-0.01890 \\
-0.01869 \\
-0.01845 \\
-0.01820 \\
-0.01794 \\
-0.03506 \\
-0.03384 \\
-0.03253 \\
-0.03114 \\
-6.05800\end{array}$ & $\begin{array}{l}\text { DCDMU } \\
0.00002 \\
0.00004 \\
0.00004 \\
0.00009 \\
0.00009 \\
0.00019 \\
0.00019 \\
0.00037 \\
0.00037 \\
0.00037 \\
0.00037 \\
0.00037 \\
0.00037 \\
0.00018 \\
0.00091 \\
0.00090 \\
0.00089 \\
0.00089 \\
0.00088 \\
0.00087 \\
0.00086 \\
2.00085 \\
0.00167 \\
0.00162 \\
0.00157 \\
0.20151 \\
0.00285\end{array}$ & $\begin{array}{l}\text { DCDRHO } \\
-0.00045 \\
-0.00091 \\
-0.00091 \\
-0.00227 \\
-0.00227 \\
-0.00452 \\
-0.00451 \\
-0.00899 \\
-0.00894 \\
-0.00889 \\
-0.00885 \\
-0.00881 \\
-0.00877 \\
-0.00437 \\
-0.02170 \\
-0.02145 \\
-0.02119 \\
-0.02091 \\
-0.02061 \\
-0.02029 \\
-0.01995 \\
-0.01958 \\
-0.03805 \\
-0.03639 \\
-0.03462 \\
-0.03277 \\
-0.05987\end{array}$ & $\begin{array}{l}\text { DCDMU } \\
0.00002 \\
0.00004 \\
0.00005 \\
0.00011 \\
0.00011 \\
0.00021 \\
0.00021 \\
0.00043 \\
0.00043 \\
6.00042 \\
0.00042 \\
0.00042 \\
0.00042 \\
0.00021 \\
0.00103 \\
0.00102 \\
0.00101 \\
0.00100 \\
0.00099 \\
0.00098 \\
0.00096 \\
1.00095 \\
0.00185 \\
0.00178 \\
0.00171 \\
0.00163 \\
0.00301\end{array}$ & $\begin{array}{l}\text { DCDRHO } \\
-0.00055 \\
-0.00110 \\
-0.00110 \\
-0.00273 \\
-0.00273 \\
-0.00545 \\
-0.00543 \\
-0.01079 \\
-0.01069 \\
-0.01059 \\
-0.01051 \\
-0.01044 \\
-0.01036 \\
-0.00515 \\
-0.02552 \\
-0.02510 \\
-0.02467 \\
-0.02422 \\
-0.02375 \\
-0.02324 \\
-0.02271 \\
-0.02214 \\
-0.04253 \\
-0.04001 \\
-0.03737 \\
-0.03464 \\
-0.06114\end{array}$ & $\begin{array}{l}\text { DCOMU } \\
0.00003 \\
0.00005 \\
0.00006 \\
0.00013 \\
0.00013 \\
0.00026 \\
0.00026 \\
0.00052 \\
0.00052 \\
0.00051 \\
0.00051 \\
0.00051 \\
0.00050 \\
0.00025 \\
0.00124 \\
0.00123 \\
0.00121 \\
0.00119 \\
0.00117 \\
0.00115 \\
0.00112 \\
0.00110 \\
0.00212 \\
0.00201 \\
0.00189 \\
0.00176 \\
0.00314\end{array}$ & $\begin{array}{l}\text { DCDRHO } \\
-0.00074 \\
-0.00148 \\
-0.00147 \\
-0.00366 \\
-0.00365 \\
-0.0 C 725 \\
-0.00720 \\
-0.01423 \\
-0.01396 \\
-0.01370 \\
-0.01351 \\
-0.01333 \\
-0.01315 \\
-0.00651 \\
-0.03197 \\
-0.03105 \\
-0.03017 \\
-0.02929 \\
-0.02839 \\
-0.02744 \\
-0.02645 \\
-0.02543 \\
-0.04777 \\
-0.04347 \\
-0.03914 \\
-0.03485 \\
-0.05758\end{array}$ & $\begin{array}{l}\text { DCDMU } \\
0.00004 \\
0.00008 \\
0.00009 \\
0.00018 \\
0.00018 \\
0.00036 \\
0.00036 \\
0.00071 \\
0.00071 \\
0.00068 \\
0.00067 \\
0.00067 \\
0.00066 \\
0.00033 \\
0.00160 \\
0.00155 \\
0.00151 \\
0.00147 \\
0.00143 \\
0.00138 \\
0.00134 \\
0.00129 \\
0.00244 \\
0.00223 \\
0.06202 \\
0.00181 \\
0.00303\end{array}$ & $\begin{array}{l}\text { DCDRHO } \\
-0.00126 \\
-0.00251 \\
-0.00248 \\
-0.00612 \\
-0.00608 \\
-0.01199 \\
-0.01175 \\
-0.02284 \\
-0.02172 \\
-0.02069 \\
-0.01996 \\
-0.01928 \\
-0.01865 \\
-0.00910 \\
-0.04355 \\
-0.04067 \\
-0.03819 \\
-0.03588 \\
-0.03369 \\
-0.03152 \\
-0.02936 \\
-0.02725 \\
-0.04848 \\
-0.04083 \\
-0.03385 \\
-0.02760 \\
-0.03974\end{array}$ & $\begin{array}{l}\text { DCDMU } \\
0.00006 \\
0.00014 \\
0.00019 \\
0.00031 \\
0.00031 \\
0.00063 \\
0.00060 \\
0.00119 \\
0.00116 \\
0.00107 \\
0.00103 \\
0.00100 \\
0.00096 \\
0.00047 \\
0.00224 \\
0.00209 \\
0.00196 \\
0.00185 \\
0.00174 \\
0.00163 \\
0.00153 \\
0.00142 \\
0.00254 \\
0.00215 \\
0.00179 \\
0.00147 \\
0.00215\end{array}$ \\
\hline \multicolumn{2}{|c|}{$\begin{array}{l}T(S E C S) \\
C(K / S E C)\end{array}$} & & \multicolumn{2}{|c|}{$\begin{array}{l}12.55 \\
4.350\end{array}$} & \multicolumn{2}{|c|}{$\begin{array}{l}11.50 \\
4.300\end{array}$} & \multicolumn{2}{|c|}{$\begin{array}{r}9.98 \\
4.250\end{array}$} & \multicolumn{2}{|c|}{$\begin{array}{r}9.23 \\
4.200\end{array}$} & \multicolumn{2}{|c|}{$\begin{array}{r}8.63 \\
4.150\end{array}$} \\
\hline
\end{tabular}

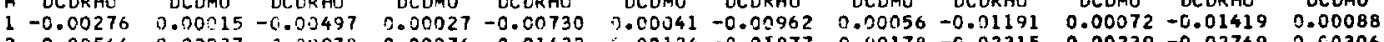

$\begin{array}{lllllllllllll}2 & -0.00546 & 0.00037 & -0.00978 & 0.00076 & -0.01432 & 0.00124 & -0.01877 & 0.00178 & -0.02315 & 0.00239 & -0.02749 & 0.00306\end{array}$

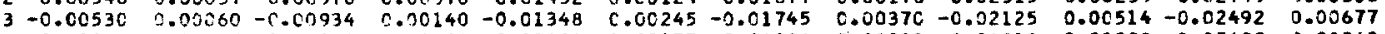

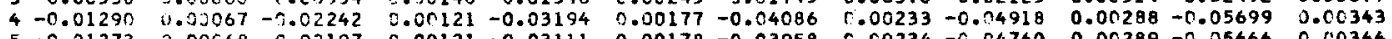

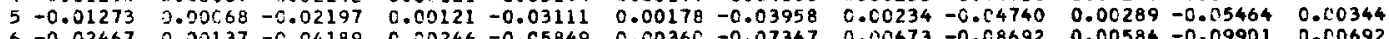

$\begin{array}{lllllllllllll}6 & -0.02467 & 0.00137 & -0.04189 & 0.00246 & -0.05849 & 0.00360 & -0.07347 & 0.00473 & -0.008692 & 0.00584 & -0.09901 & 0.00692\end{array}$

$\begin{array}{lllllllllllll}7 & -0.02360 & 0.00127 & -0.03912 & 0.00219 & -0.05351 & 0.00311 & -0.065597 & 0.00398 & -0.007667 & 0.00478 & -0.008582 & 0.00 \times 554\end{array}$

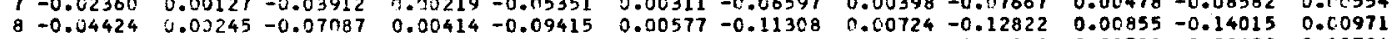

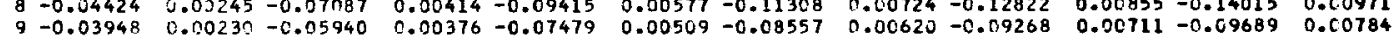

$10-0.03530 \quad 0.00192-0.04979 \quad 0.00286-0.05918 \quad 0.00356-0.06418 \quad 0.00405-0.06600 \quad 0.00435-0.06554 \quad 0.00451$

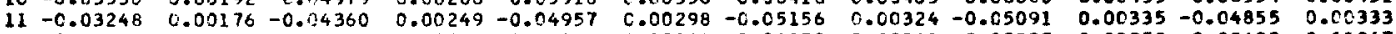

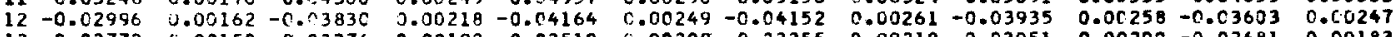

$13-0.02772 \quad 0.00150-0.03376 \quad 0.00192-0.0351 \mathrm{C} \quad 0.00209-0.03355 \quad 0.00210-0.03051 \quad 0.00200-0.02681 \quad 0.00183$

$14-0.01309 \quad 0.00071-0.01538 \quad 0.00387-0.01546 \quad 0.00092-0.01431 \quad 0.00089-0.01261 \quad 0.00082-0.01073 \quad 0.00073$

$\begin{array}{llllllllllllll}15 & -0.05902 & 0.00317 & -0.06484 & 0.00365 & -0.06125 & 0.00361 & -0.05345 & 0.00330 & -0.044448 & 0.00288 & -0.03578 & 0.00242\end{array}$

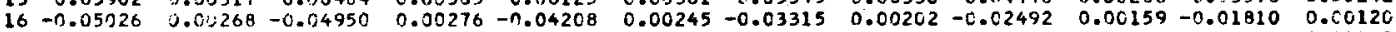

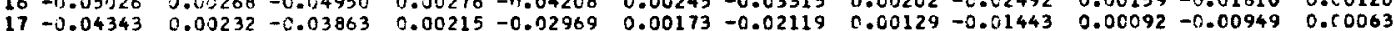

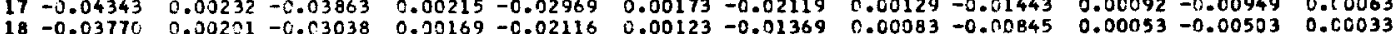

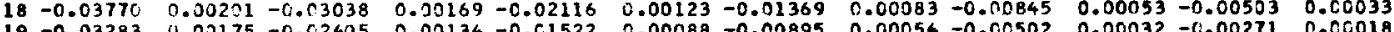

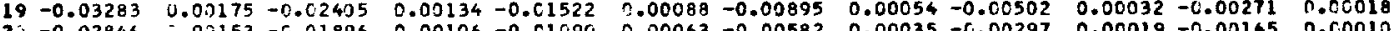

$25=0.02846 \quad 5.00153-0.01896 \quad 0.00106-0.01090 \quad 0.00063-0.00582 \quad 0.00035-0.00297 \quad 0.00019-0.00145 \quad 0.0 .0010$

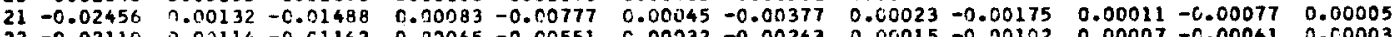

$22-0.02110 \quad 0.00114-0.01162 \quad 0.00065-0.00551 \quad 0.00032-0.00243 \quad 0.00015-0.00102 \quad 0.00007-0.00041 \quad 0.00003$

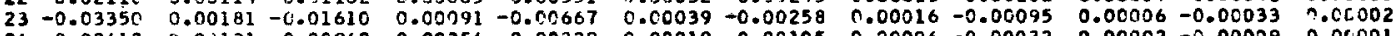

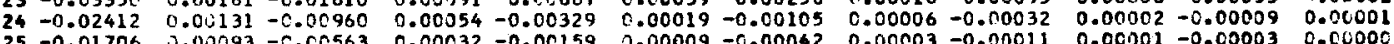

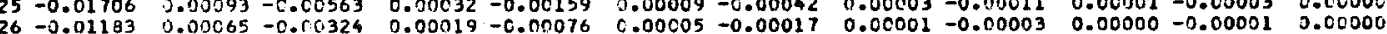

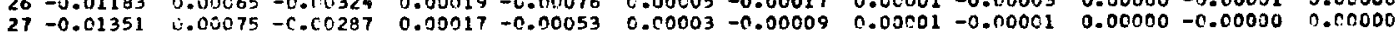

As an early test of the method the dispersion and partials were computed for a Jeffreys-Bullen type mantle. These results were used to predict the dispersion when the layer parameters were modified in such a way as to approximate a GutenbergBullen type mantle. The actual dispersion for a Gutenberg-Bullen model was then computed. Since the partial derivative tables are routine computer output in the 
TABLE $10 \mathrm{~b}$

Variational Partial Derivatives for CIT13F Laminated Crust and Upper Mantle

Ochanic Earth Model

CIT $13 \mathrm{~F}$ OCEANIC LAMINATED CRUST AND UPPER MANTLE

\begin{tabular}{|c|c|c|c|c|c|c|c|c|c|c|c|c|}
\hline \multicolumn{3}{|c|}{$\begin{array}{l}\text { T(SECS) } \\
C(K / \text { SEC })\end{array}$} & \multicolumn{2}{|c|}{$\begin{array}{r}7.68 \\
4.050\end{array}$} & \multicolumn{2}{|c|}{$\begin{array}{r}7.29 \\
4.000\end{array}$} & \multicolumn{2}{|c|}{$\begin{array}{r}6.94 \\
3.950\end{array}$} & \multicolumn{2}{|c|}{$\begin{array}{r}6.62 \\
3.900\end{array}$} & \multicolumn{2}{|c|}{$\begin{array}{r}6.32 \\
3.850\end{array}$} \\
\hline $\begin{array}{l}M \\
1 \\
2 \\
3 \\
4 \\
5 \\
6 \\
7 \\
8 \\
9 \\
10 \\
11 \\
12 \\
13 \\
14 \\
15 \\
16 \\
17 \\
18 \\
19 \\
20 \\
21 \\
22\end{array}$ & $\begin{array}{l}\text { OCDRHO } \\
-0.01650 \\
-0.03183 \\
-0.02848 \\
-0.06436 \\
-0.06138 \\
-0.10991 \\
-0.09360 \\
-0.14931 \\
-0.09880 \\
-0.06346 \\
-0.04512 \\
-0.03213 \\
-0.02293 \\
-0.00889 \\
-0.02801 \\
-0.01277 \\
-0.00605 \\
-0.00289 \\
-0.00141 \\
-0.00068 \\
-0.00033 \\
-0.00016\end{array}$ & $\begin{array}{l}\text { DCDMU } \\
0.00106 \\
0.00380 \\
0.00863 \\
0.01398 \\
0.00399 \\
0.00799 \\
0.00624 \\
0.01074 \\
0.00841 \\
0.00455 \\
0.00323 \\
0.00230 \\
0.00164 \\
0.00063 \\
0.00198 \\
0.00089 \\
0.00042 \\
0.00020 \\
0.0001 C \\
0.00005 \\
0.00002 \\
0.00001\end{array}$ & $\begin{array}{l}\text { DCDRHO } \\
-0.01885 \\
-0.03622 \\
-0.03198 \\
-0.07135 \\
-0.06768 \\
-0.11973 \\
-0.10016 \\
-0.15605 \\
-0.09885 \\
-0.06023 \\
-0.04106 \\
-0.02803 \\
-0.01917 \\
-0.00719 \\
-0.02140 \\
-0.00877 \\
-0.00374 \\
-0.00161 \\
-0.00071 \\
-0.00031 \\
-0.00013 \\
-0.00006\end{array}$ & $\begin{array}{l}\text { DCDMU } \\
0.00124 \\
0.00463 \\
0.01073 \\
0.00454 \\
0.00454 \\
0.00905 \\
0.00690 \\
0.01163 \\
0.00882 \\
0.00450 \\
0.00307 \\
0.00209 \\
0.00143 \\
0.00053 \\
0.00158 \\
0.00564 \\
0.00027 \\
0.00012 \\
0.00005 \\
0.00002 \\
0.00001 \\
0.00000\end{array}$ & $\begin{array}{l}\text { DCDRHO } \\
-0.02127 \\
-0.04069 \\
-0.03543 \\
-0.07800 \\
-0.07357 \\
-0.12853 \\
-0.10556 \\
-0.16060 \\
-0.09735 \\
-0.05619 \\
-0.03668 \\
-0.02397 \\
-0.01569 \\
-0.00568 \\
-0.01597 \\
-0.00586 \\
-0.00224 \\
-0.00087 \\
-0.00034 \\
-0.00013 \\
-0.00005 \\
-0.00002\end{array}$ & $\begin{array}{l}\text { OCOMU } \\
0.00145 \\
0.00557 \\
0.01312 \\
0.00511 \\
0.00509 \\
0.01009 \\
0.00751 \\
0.01239 \\
0.00909 \\
0.00437 \\
0.00286 \\
0.00186 \\
0.00122 \\
0.00044 \\
0.00123 \\
0.00044 \\
0.00017 \\
0.00006 \\
0.00003 \\
0.00001 \\
0.00000 \\
0.00000\end{array}$ & $\begin{array}{l}\text { DCDRHO } \\
-0.02379 \\
-0.04529 \\
-0.03886 \\
-0.08434 \\
-0.07908 \\
-0.13639 \\
-0.10988 \\
-0.16317 \\
-0.09459 \\
-0.05162 \\
-0.03222 \\
-0.02013 \\
-0.01259 \\
-0.00440 \\
-0.01165 \\
-0.00381 \\
-0.00130 \\
-0.00045 \\
-0.00016 \\
-0.00006 \\
-0.00002 \\
-0.00001\end{array}$ & $\begin{array}{l}\text { DCDHU } \\
0.00167 \\
0.00662 \\
0.01583 \\
0.00569 \\
0.00564 \\
0.01112 \\
0.00807 \\
0.01302 \\
0.00922 \\
0.00419 \\
0.00261 \\
0.00163 \\
0.00102 \\
0.00036 \\
0.00093 \\
0.00030 \\
0.00010 \\
0.00004 \\
0.00001 \\
0.00000 \\
0.00000 \\
0.00000\end{array}$ & $\begin{array}{l}\text { DCDRHO } \\
-0.02644 \\
-0.05008 \\
-0.04231 \\
-0.09041 \\
-0.08425 \\
-0.14334 \\
-0.11317 \\
-0.16393 \\
-0.09081 \\
-0.04676 \\
-0.02785 \\
-0.01659 \\
-0.00990 \\
-0.00333 \\
-0.00831 \\
-0.00241 \\
-0.00073 \\
-0.00022 \\
-0.00007 \\
-0.00002 \\
-0.00001 \\
-0.00000\end{array}$ & $\begin{array}{l}\text { DCDMU } \\
0.00192 \\
0.00782 \\
0.01894 \\
0.00628 \\
0.00621 \\
0.01214 \\
0.00859 \\
0.01352 \\
0.00923 \\
0.00395 \\
0.00235 \\
0.00140 \\
0.00083 \\
0.00028 \\
0.00069 \\
0.00020 \\
0.00006 \\
0.00002 \\
0.00001 \\
0.00000 \\
0.00000 \\
0.00000\end{array}$ & $\begin{array}{l}\text { DCORHO } \\
-0.02925 \\
-0.05512 \\
-0.04579 \\
-0.09624 \\
-0.00908 \\
-0.14942 \\
-0.11549 \\
-0.16302 \\
-0.08620 \\
-0.04178 \\
-0.02369 \\
-0.01343 \\
-0.00762 \\
-0.00247 \\
-0.00578 \\
-0.00148 \\
-0.00040 \\
-0.00011 \\
-0.00003 \\
-0.00001 \\
-0.00000 \\
-0.00000\end{array}$ & $\begin{array}{l}\text { DCDMU } \\
0.00219 \\
0.00919 \\
0.02250 \\
0.00688 \\
0.00678 \\
0.01314 \\
0.00905 \\
0.01389 \\
0.00912 \\
0.00367 \\
0.00208 \\
0.00118 \\
0.00067 \\
0.00022 \\
0.00050 \\
0.00013 \\
0.00003 \\
0.00001 \\
0.00000 \\
0.00000 \\
0.00000 \\
0.00000\end{array}$ \\
\hline & & & & & & & & & & & & \\
\hline
\end{tabular}

\begin{tabular}{|c|c|c|c|c|c|c|c|c|c|c|c|c|}
\hline 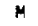 & DCDRHO & $\operatorname{ccDN} \mathrm{J}$ & LCDRHO & OCENU & ECDRHO & COM & CCORHO & DCQMU & DCDRHO & DCDMU & DCORHO & DCDMU \\
\hline 1 & $\begin{array}{l}-0.03228 \\
-0.06050\end{array}$ & $\begin{array}{l}0.00249 \\
0.01076\end{array}$ & $\begin{array}{l}-0.03558 \\
-0.06629\end{array}$ & $\begin{array}{l}0.00284 \\
0.01258\end{array}$ & $\begin{array}{l}-0.03922 \\
-0.07262\end{array}$ & $\begin{array}{l}0.00324 \\
0.01471\end{array}$ & $\begin{array}{l}-0.04328 \\
-0.07961\end{array}$ & $\begin{array}{l}0.00370 \\
0.01721\end{array}$ & $\begin{array}{l}-0.04787 \\
-0.08741\end{array}$ & $\begin{array}{l}0.00423 \\
0.02018\end{array}$ & $\begin{array}{l}-0.05310 \\
-0.09621\end{array}$ & $\begin{array}{l}0.00486 \\
0.02371\end{array}$ \\
\hline 3 & -0.04936 & .02661 & -0.05305 & .03130 & -0.05691 & 0.03655 & -0.06099 & .04350 & -0.06535 & 0.05123 & -0.07006 & 0.06040 \\
\hline 4 & -0.10187 & 0.00751 & -0.10732 & 0.00816 & $-\mathrm{c} .11263$ & $0 . \cos 84$ & -0.11783 & 0.00955 & -0.12295 & 0.01030 & -0.12801 & 0.01109 \\
\hline 5 & -0.0 & c. 00736 & -0.09787 & 0.00795 & -0.10187 & 0.00856 & $-0.1 \mathrm{CS} 61$ & 0.00919 & -0.10913 & 0.00983 & -0.11240 & 0.01050 \\
\hline 6 & -0.15467 & 0.01413 & -0.15911 & 0.01511 & -0.16278 & 0.01607 & $-0.1 \in 568$ & 0.01702 & -0. & 795 & -0 & 87 \\
\hline 7 & -0.11687 & 0.00946 & -0.11736 & 0.00982 & -0.11698 & 0.01013 & -0.11578 & 0.01037 & -0.11380 & 56 & -0.1 & 0.01068 \\
\hline 8. & -0.1 & 0.01414 & -0.15679 & 0.01425 & -0.15174 & 0.01424 & -0.14556 & 0.01411 & -0.13841 & 86 & -0. & 0.01350 \\
\hline 9 & $-C .08096$ & 0.00891 & -0.07523 & 0.00860 & -0.06915 & 0.00820 & -0.06296 & 0.00773 & -0.05667 & 0.00720 & -0.0 & 0. \\
\hline 10 & -0.03683 & 0.00337 & -0.03203 & 0.00305 & -0.02748 & C. C0272 & -0.02325 & 0.00240 & -0.01939 & 0.00208 & -0. & 0. \\
\hline 1 & -0.0 & 0.00181 & -0.01633 & 0.00 & -0.01323 & 0. & -0.01053 & 109 & -0.0 & 88 & -0. & 0. \\
\hline 12 & -0.0 & 0.0 & -0.00832 & 0.00 & -0.00636 & $0 . \mathrm{Co}$ & -0.00477 & 0.09 & -0.0 & 038 & -0. & \\
\hline 13 & -0.00575 & 0.00053 & $-c .00424$ & 0.00040 & -0.00306 & 0.00030 & -0.00216 & 0.00022 & -0.00 & 016 & -0.00 & 0.00 \\
\hline 14 & $=0.00179$ & c. $\operatorname{coc} 1 t$ & $=0.00126$ & C. $0 \mathrm{CO} 12$ & -0.00087 & 0.00009 & -0.00058 & 0.00006 & -0.00038 & 104 & -0.00024 & 0.00003 \\
\hline 15 & -0.00393 & $0 . \operatorname{coc} 36$ & $-c . c 0260$ & $0.0 \mathrm{C}$ & -0.00167 & 0.00 & -0.00105 & 0.00011 & -0.0 & 0.0 & -0. & 0 . \\
\hline 16 & -0.00088 & C.c0008 & -0.00051 & $0 . \operatorname{cccos}$ & -0.00028 & $\mathrm{c} . \mathrm{co}$ & -0.00015 & 0.00 & $-0 . \operatorname{cocos}$ & $0 . c$ & -0 & 0. \\
\hline 17 & -0.00021 & $0 . \mathrm{COCO} 2$ & $-c . c 0010$ & 0.00001 & -0.00005 & 0.00000 & $-0 . \operatorname{ccco} 2$ & 0.00000 & -0.00001 & 0.10000 & -0.00000 & 0.0 \\
\hline 18 & -0.00005 & $0 . \operatorname{cccco}$ & $-0 . \operatorname{cco0} 2$ & 0.00000 & -0.00001 & $0 . \operatorname{coc} 0$ & $-0.0 \mathrm{c} \cap 00$ & $0.0 \mathrm{COOOO}$ & $-0 . \operatorname{ccoco}$ & 0.00000 & -0.00000 & 0.00 \\
\hline 19 & -0 & $0 . \mathrm{C}$ & $-0 . c$ & 0.0 & $-c$. & & -0 & 0.0 & -0 . & 0.0 & 00 & \\
\hline 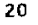 & & 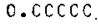 & & 0.00 & -0.0 & 0.00 & 60 & 0.00000 & 0000 & .00000 & 000 & 0.00000 \\
\hline 1 & 2 & $\begin{array}{l}70 \\
60\end{array}$ & & & & & & & & Ju & & $\begin{array}{l}2 \\
0\end{array}$ \\
\hline
\end{tabular}

$\begin{array}{lr}\text { T(SECS) } & 4.70 \\ \text { C(K/SEC) } & 3.500\end{array}$

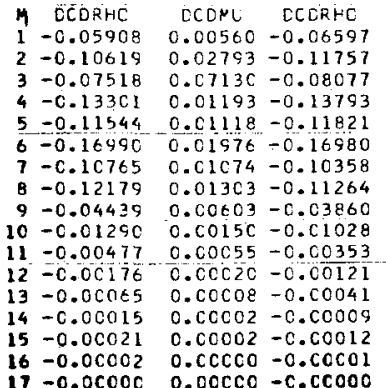

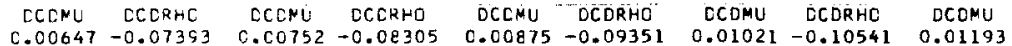

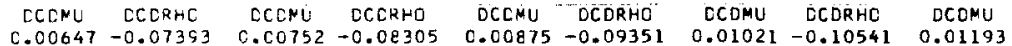

DCOMU $\begin{array}{lllllllll}0.00647 & -0.07393 & 0.00752 & -0.08305 & 0.00875 & -0.09351 & 0.01021 & -0.10541 & 0.01193 \\ 0.03298 & -0.13058 & 0.03903-0.14534 & 0.04623 & -0.1621 C & 0.05476 & -0.18099 & 0.06480\end{array}$ $\begin{array}{llllllll}c .08427-0.08691 & 0.09969-0.09356 & 0.11788 & -0.1008 \mathrm{C} & 0.13925 & -0.10862 & 0.16414\end{array}$

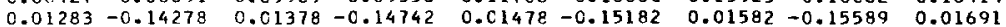
$\begin{array}{lllllllll}0.01189 & -0.12071 & 0.01262 & -0.12282 & 0.01336 & -0.12451 & 0.01410 & -0.12574 & 0.01484\end{array}$ $\begin{array}{lllllllll}0.02061 & -0.16896 & 0.02143 & -0.16727 & 0.02219 & 0.0 .16477 & 0.02288 & -0.16146 & 0.02347\end{array}$ $0.01073-0.098960 .01085-0.093790 .01050-0.088210 .01028-0.082310 .01000$

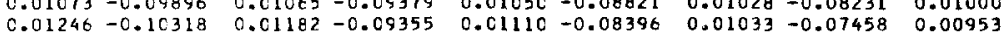

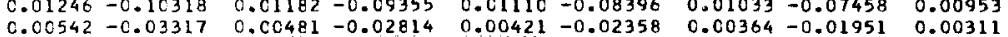
$\begin{array}{lllllllll}0.00542 & -0.03317 & 0.00481 & -0.02814 & 0.00421 & -0.02358 & 0.00364 & -0.01951 & 0.00311 \\ 0.00124 & -0.00808 & 0.00102 & -0.06624 & 0.00082 & -0.00476 & 0.00065 & -0.00357 & 0.00051\end{array}$ $\begin{array}{lllllllll}0.00124 & -0.00808 & 0.00102 & -0.00624 & 0.00082 & -0.00476 & 0.00065 & -0.00357 & 0.00051 \\ 0.00043 & -0.00256 & 0.00032 & -0.00183 & 0.00024 & -0.00128 & 0.00017 & -0.00088 & 0.00013\end{array}$

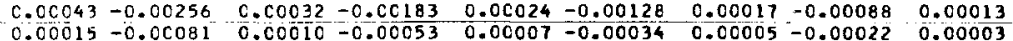

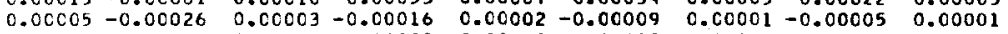
$\begin{array}{llllllll}0.00001-0.00005 & 0.0 C C C l & -0.00 C 03 & 0.00000 & -0.00002 & 0.00000 & -0.00001 & 0.00000\end{array}$ $\begin{array}{lllllllllll}c .00001 & -0.00006 & 0.00001 & -0.06 C 03 & 0.00000 & -0.06002 & 0.00000 & -0.00001 & 0.00000\end{array}$ $0.00000-0.00000 \quad 0.00 C 00-0.00000 \quad 0.00000-0.06000 \quad 0.00000-0.00000 \quad 0.00000$ $0.00000-0.00000 \quad 0.0 \operatorname{coc} 0-0.06000 \quad 0.06000=0.06000 \quad 0.00000-0.000000 .00000$

Caltech dispersion programs the new tables were used to predict back to the JeffreysBullen model. The results are shown in fig. 1.

The tables are particularly designed for investigation of the physical properties of the crust and mantle. However, the effect of crustal thickness changes may be 
estimated by use of equation 17 . Figure 2 shows a rather extreme trial case where another forward-backward experiment has been performed for illustration. In the "forward" case a crustal layer has been decreased in thickness by $50 \%$. In the

TABLE 11a

Phase Velocity Perturbations (km/sec) Resulting from a $10 \%$ Change in Density and Rigidity for CIT13F Laminated Crost and Upper Mantle Oceanic Earth Model

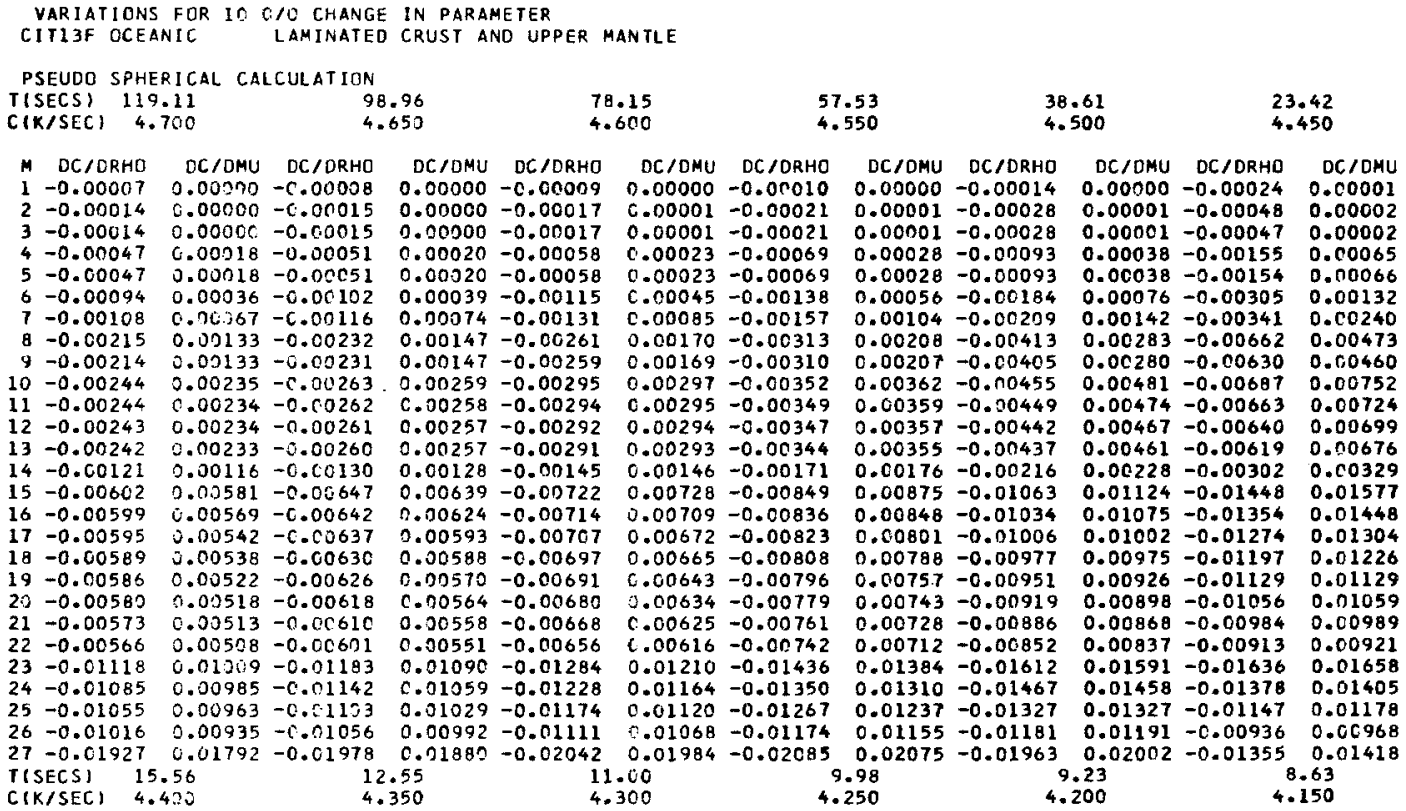

\begin{tabular}{|c|c|c|c|c|c|c|c|c|c|c|c|}
\hline $\begin{array}{l}-0.0 \\
-0.0 \\
-0.0 \\
-0.0 \\
-0.0 \\
-0.1 \\
-0.0 \\
-0.0 \\
-0.0 \\
-0.0 \\
-0.0 \\
-0.0 \\
-0 . \\
-0 . \\
-0 . \\
-0 . \\
-0 . \\
-0 . \\
-0 .\end{array}$ & $\begin{array}{l}.01138 \\
.00989 \\
.00856 \\
.00738 \\
.011185 \\
.00859 \\
.00614 \\
.00429 \\
.00497\end{array}$ & $\begin{array}{l}-0.01 \\
-0.02 \\
-0.01 \\
-0.01 \\
-6.01 \\
-0.01 \\
-0.01 \\
-0.00 \\
-0.02 \\
-0.01 \\
-0.01 \\
-0.01 \\
-6.00 \\
-0.00 \\
-0.00 \\
-0.00 \\
-0.00 \\
-c .00 \\
-0.00 \\
-0.00 \\
-0.00\end{array}$ & $\begin{array}{l}43 \\
9 \\
7 \\
54 \\
56 \\
8 \\
10 \\
4 \\
5 \\
6 \\
33 \\
35 \\
8 \\
12 \\
58 \\
12 \\
26 \\
19 \\
58 \\
36 \\
40 \\
23 \\
93 \\
56 \\
11\end{array}$ & $\begin{array}{l}-0 . \\
-0 . \\
-0 . \\
-0 . \\
-0 . \\
-0 . \\
-0 . \\
-0 . \\
-0 . \\
-0 . \\
-0 . \\
-0 . \\
-0 . \\
-0 . \\
-0 . \\
-0 . \\
-0 . \\
-0 . \\
-0 . \\
-0 . \\
-0 . \\
-0 .\end{array}$ & $\begin{array}{l}0 \\
5 \\
5 \\
0 \\
3 \\
5 \\
9 \\
6 \\
2 \\
1 \\
4 \\
2 \\
2 \\
2 \\
2 \\
6 \\
3 \\
9 \\
5 \\
3 \\
2 \\
1 \\
14 \\
19 \\
6 \\
7 \\
2\end{array}$ & $\begin{array}{l}-0 . \\
-0 . \\
-0 . \\
-0 . \\
-0 . \\
-0 . \\
-0 . \\
-0 . \\
-0 . \\
-0 . \\
-0 . \\
-0 . \\
-0 . \\
-0 . \\
-0 . \\
-0 . \\
-0 . \\
-0 . \\
-0 . \\
-0 . \\
-0 . \\
-0 . \\
-0 . \\
-0 . \\
-0 .\end{array}$ & $\begin{array}{l}\text { DCfOMU } \\
0.00007 \\
0.00022 \\
0.00045 \\
0.00490 \\
0.00493 \\
0.00997 \\
0.01579 \\
0.02875 \\
0.02462 \\
0.02842 \\
0.02279 \\
0.01831 \\
0.01474 \\
0.00627 \\
0.02324 \\
0.01399 \\
0.00854 \\
0.00549 \\
0.00351 \\
0.00229 \\
0.00149 \\
0.00096 \\
0.00103 \\
0.00042 \\
0.00017 \\
0.00007 \\
0.00004\end{array}$ & $\begin{array}{l}-0 \\
-0 \\
-0 \\
-0 \\
-0 \\
-0 \\
-0 \\
-0 \\
-0 \\
-0 \\
-0 \\
-0 \\
-0 \\
-0 \\
-0 \\
-0 \\
-0 \\
-0 \\
-0 \\
-0 \\
-0 \\
-0 \\
-0\end{array}$ & & $\begin{array}{l}10 \\
22 \\
73 \\
8 \\
88 \\
15 \\
39 \\
54 \\
10 \\
76 \\
12 \\
96 \\
90 \\
56 \\
90 \\
03 \\
16 \\
58 \\
91 \\
49 \\
26 \\
44 \\
11 \\
53 \\
01 \\
00\end{array}$ & 0.000 \\
\hline
\end{tabular}

"backward" case the thickness has been increased back to the original value-this corresponding now to a $100 \%$ change in thickness. It is more accurate to modify the physical properties in the framework of the standard model layering as also shown in figure 2. This tactic removes the restriction of small changes since the maximum 
TABLE $11 \mathrm{~b}$

Phase Velocity Perturbations (km/sec) Resulting from a 10\% Change in Density and Rigidity for Cit13F Laminated Crust and Upper Mantle Oceanic Earth Model

CIT $13 F$ OCEANIC LAMINATED CRUST AND UPPER MANTLE

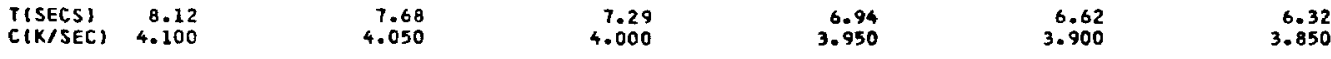

\begin{tabular}{|c|c|c|c|c|c|c|c|c|c|c|c|c|}
\hline $\begin{array}{l}1 \\
1 \\
2 \\
3 \\
4 \\
5 \\
6 \\
7 \\
8 \\
9 \\
0\end{array}$ & $\begin{array}{l}\text { DC/DRHO } \\
-0.00314 \\
-0.00605 \\
-0.00541 \\
-0.01635 \\
-0.01559 \\
-0.02792 \\
-0.02715 \\
-0.04330 \\
-0.02865 \\
-0.02107 \\
-0.01498 \\
-0.01067 \\
-0.00761 \\
-0.00295 \\
-0.00931 \\
-0.00425 \\
-0.00202 \\
-0.00096 \\
-0.00047\end{array}$ & $\begin{array}{l}\text { DC/DMU } \\
0.00013 \\
0.00046 \\
0.00105 \\
0.00839 \\
0.00840 \\
0.01684 \\
0.02477 \\
0.04262 \\
0.03338 \\
0.03197 \\
0.02271 \\
0.01615 \\
0.01150 \\
0.00444 \\
0.01391 \\
0.00614 \\
0.00277 \\
0.00132 \\
0.00063\end{array}$ & $\begin{array}{r}\text { DC/DRHO } \\
-0.00358 \\
-0.00688 \\
-0.00608 \\
-0.01812 \\
-0.01719 \\
-0.03041 \\
-0.02905 \\
-0.04526 \\
-0.02867 \\
-0.02000 \\
-0.01363 \\
-0.00931 \\
-0.00636 \\
-0.00239 \\
-0.00711 \\
-0.00292 \\
-0.00125 \\
-0.00054 \\
-0.00024\end{array}$ & $\begin{array}{l}0 C / D M U \\
0.00015 \\
0.00056 \\
0.00130 \\
0.00957 \\
0.00956 \\
0.01907 \\
0.02738 \\
0.04616 \\
0.03502 \\
0.03162 \\
0.02155 \\
0.01469 \\
0.01003 \\
0.00375 \\
0.01109 \\
0.00440 \\
0.00179 \\
0.00077 \\
0.00033\end{array}$ & $\begin{array}{l}D C / D R H O \\
-0.00404 \\
-0.00773 \\
-0.00673 \\
-0.01981 \\
-0.01869 \\
-0.03265 \\
-0.03061 \\
-0.04657 \\
-0.02823 \\
-0.01865 \\
-0.01218 \\
-0.00796 \\
-0.00521 \\
-0.00189 \\
-0.00531 \\
-0.00195 \\
-0.00075 \\
-0.00029 \\
-0.00011\end{array}$ & $\begin{array}{l}\text { DC/DMU } \\
0.00018 \\
0.00068 \\
0.00160 \\
0.01076 \\
0.01072 \\
0.02126 \\
0.02980 \\
0.04917 \\
0.03609 \\
0.03073 \\
0.02006 \\
0.01310 \\
0.00856 \\
0.00309 \\
0.00864 \\
0.00307 \\
0.00112 \\
0.00043 \\
0.00017\end{array}$ & $\begin{array}{l}\text { OC/ORHO } \\
-0.00452 \\
-0.00861 \\
-0.00738 \\
-0.02142 \\
-0.02009 \\
-0.03464 \\
-0.03186 \\
-0.04732 \\
-0.02743 \\
-0.01714 \\
-0.01070 \\
-0.00668 \\
-0.00418 \\
-0.00146 \\
-0.00388 \\
-0.00127 \\
-0.00043 \\
-0.00015 \\
-0.00005\end{array}$ & $\begin{array}{l}\text { DC/OMU } \\
0.00020 \\
0.00081 \\
0.00193 \\
0.01198 \\
0.01189 \\
0.02343 \\
0.03204 \\
0.05168 \\
0.03662 \\
0.02941 \\
0.01835 \\
0.01146 \\
0.00716 \\
0.00250 \\
0.00657 \\
0.00208 \\
0.00068 \\
0.00023 \\
0.00008\end{array}$ & $\begin{array}{r}\text { DC/ORHO } \\
-0.00502 \\
-0.00952 \\
-0.00004 \\
-0.02296 \\
-0.02140 \\
-0.03641 \\
-0.03282 \\
-0.04754 \\
-0.02634 \\
-0.01552 \\
-0.00925 \\
-0.00551 \\
-0.00329 \\
-0.00111 \\
-0.00276 \\
-0.00080 \\
-0.00024 \\
-0.00007 \\
-0.00002\end{array}$ & $\begin{array}{l}\text { OC/DMU } \\
0.00023 \\
0.00095 \\
0.00230 \\
0.01322 \\
0.01308 \\
0.02557 \\
0.03409 \\
0.05367 \\
0.03666 \\
0.02773 \\
0.01652 \\
0.00984 \\
0.00586 \\
0.00197 \\
0.00688 \\
0.00137 \\
0.00040 \\
0.00012 \\
0.00004\end{array}$ & $\begin{array}{l}\text { DC/DRHO } \\
-0.00556 \\
-0.01047 \\
-0.00870 \\
-0.02444 \\
-0.02263 \\
-0.03795 \\
-0.03349 \\
-0.04727 \\
-0.02500 \\
-0.01387 \\
-0.00786 \\
-0.00446 \\
-0.00253 \\
-0.00082 \\
-0.00192 \\
-0.00049 \\
-0.00013 \\
-0.00004 \\
-0.00001\end{array}$ & $\begin{array}{r}D C / 0 M U \\
0.00027 \\
0.00112 \\
0.00274 \\
0.01450 \\
0.01428 \\
0.02768 \\
0.03593 \\
0.05515 \\
0.03623 \\
0.02579 \\
0.01463 \\
0.00829 \\
0.00470 \\
0.00152 \\
0.00354 \\
0.00088 \\
0.00022 \\
0.00006 \\
0.00002\end{array}$ \\
\hline & 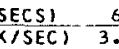 & & & & & & & & & & & \\
\hline
\end{tabular}

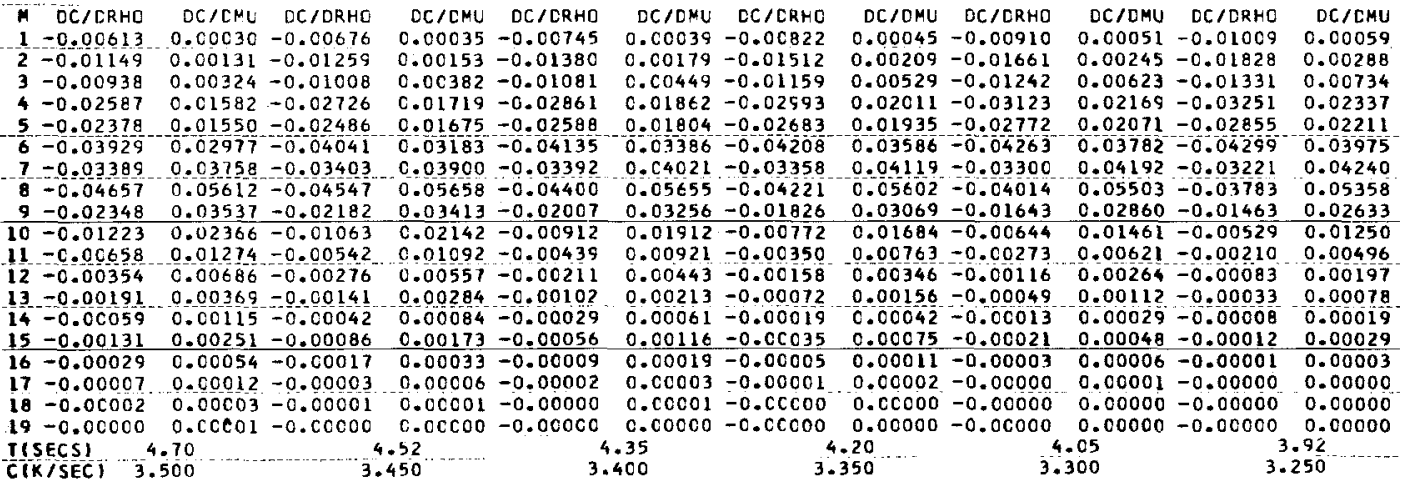

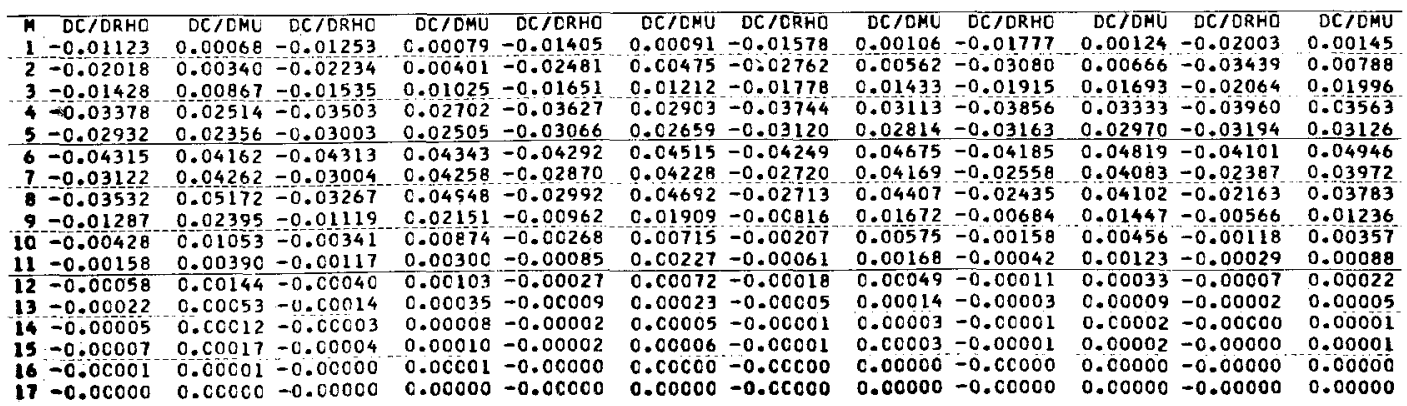

change is from a mantle velocity or density to the corresponding crustal value or vice versa.

\section{Partial Derivative Curves}

The tables of the preceding section allow for the effects of sphericity and a liquid core and can be used to calculate dispersion over any earth model. When plotted 


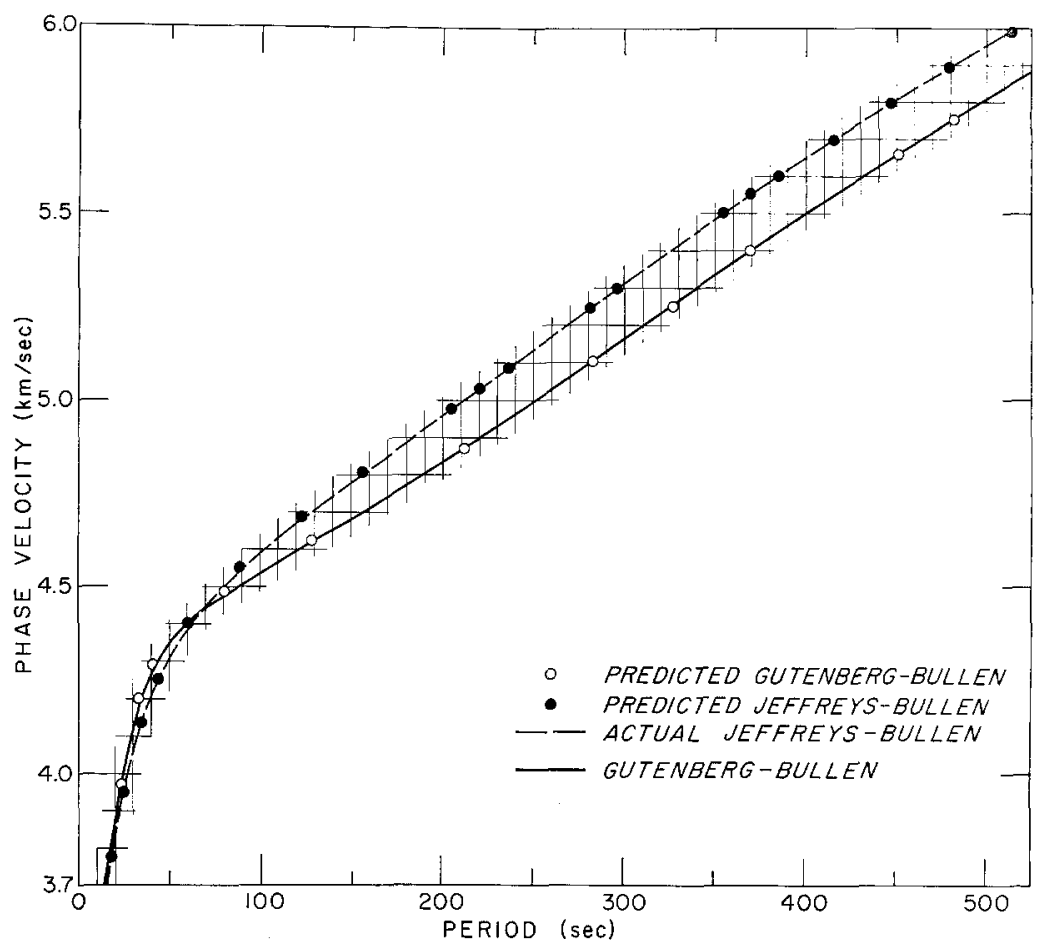

FIG. 1. Results of a numerical experiment in which the known dispersion results and partial derivative tables for a Jeffreys-Bullen type earth model are used to predict dispersion for a model with a Gutenberg-Bullen upper mantle and vice versa.

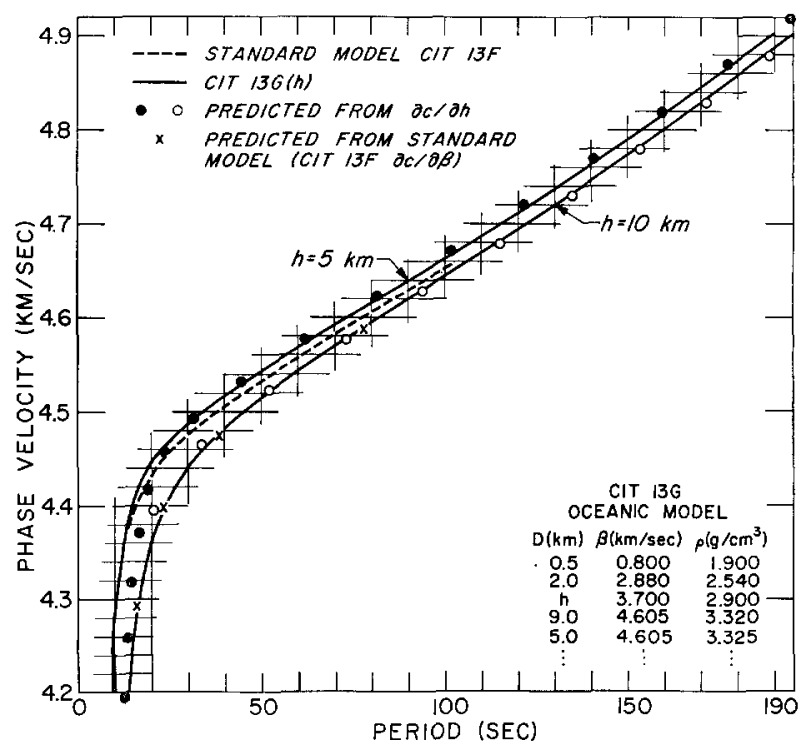

Frg. 2. Results of numerical experiment using equation 17 to predict effect of a change in thickness of a crustal layer compared with results obtained by modifying the shear velocities in the upper $250 \mathrm{~km}$. of the standard model CIT13F so as to correspond to the desired structure. 
the resulting partial derivative curves shed considerable light on the meaning and interpretation of dispersion data. The partial derivative curves tend to be bell shaped (on semi-log and log-log graphs) and peak at periods corresponding to horizontal wave lengths of roughly 2 to 6 times the layer depth. The peak, or maximum effect, decreases with increasing depth of the layer for a layer of a given thickness. The near surface layers dominate over quite a wide frequency range and their properties must be well known before any attempt is made to analyze the deeper layers, i.e., structures should be designed from the top down.

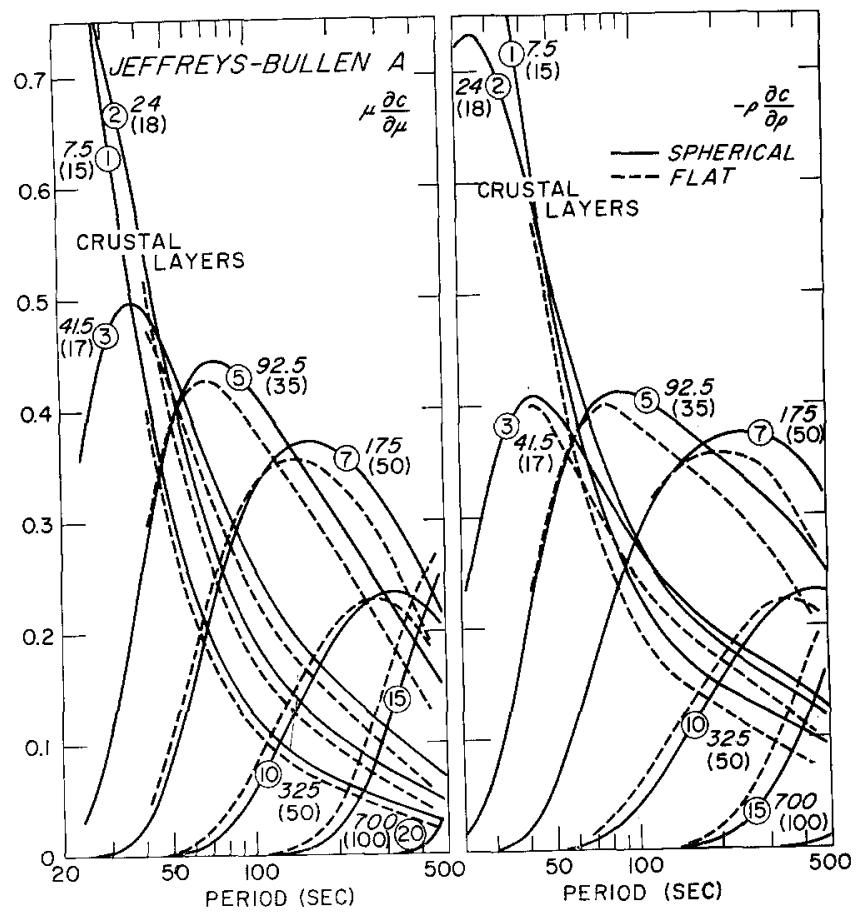

Fig. 3. Phase velocity perturbations ( $\mathrm{km} / \mathrm{sec}$ ) for Jeffreys-Bullen A Earth Model. The parameters are layer number (circled), depth to layer midpoint (in $\mathrm{km}$.) and layer thickness (in parentheses). To use, multiply the ordinate by the fractional change of the appropriate variable. Attenuation can be computed by allowing the perturbations to become complex.

These curves may be used to determine which section of the waveguide is contributing to an observed set of dispersion data and which sections are beyond the reach of the observations. They can be used to design a structure which satisfies the data and indicate how much freedom can be taken with the resulting model without violating the data to more than the experimental error.

Figure 3 shows partial derivative curves for the Jeffreys-Bullen Continental Model. In this model the rigidity and density increase monotonically with depth. Since the displacements for such a model die off approximately exponentially with depth, the partial derivative curves are roughly symmetrical on a logarithmic period scale and the peak effect divided by the layer thickness dies off uniformly with layer depth. Also shown are the partial derivatives for the equivalent flat layered model. Note that the effect of sphericity is to shift the curves to longer periods and slightly 
reduce the symmetry. These curves may be normalized by dividing by the layer thickness.

Corresponding curves for the Gutenberg IV continental model (MacDonald and Ness, 1961) are shown in figure 4 . This model has a velocity reversal in the upper mantle and the possibility exists for efficient channeling of certain periods. This effect shows up in a skewing of the partial derivative curves toward shorter periods.

The oceanic model CIT 9 has a more pronounced low-velocity zone and the skewing in figures 5 and 6 is even more apparent. An additional phenomenon, that of a cut-off period, also shows up now. A low rigidity region partially decouples the crust from the mantle. At periods greater than the decoupling period, which in this

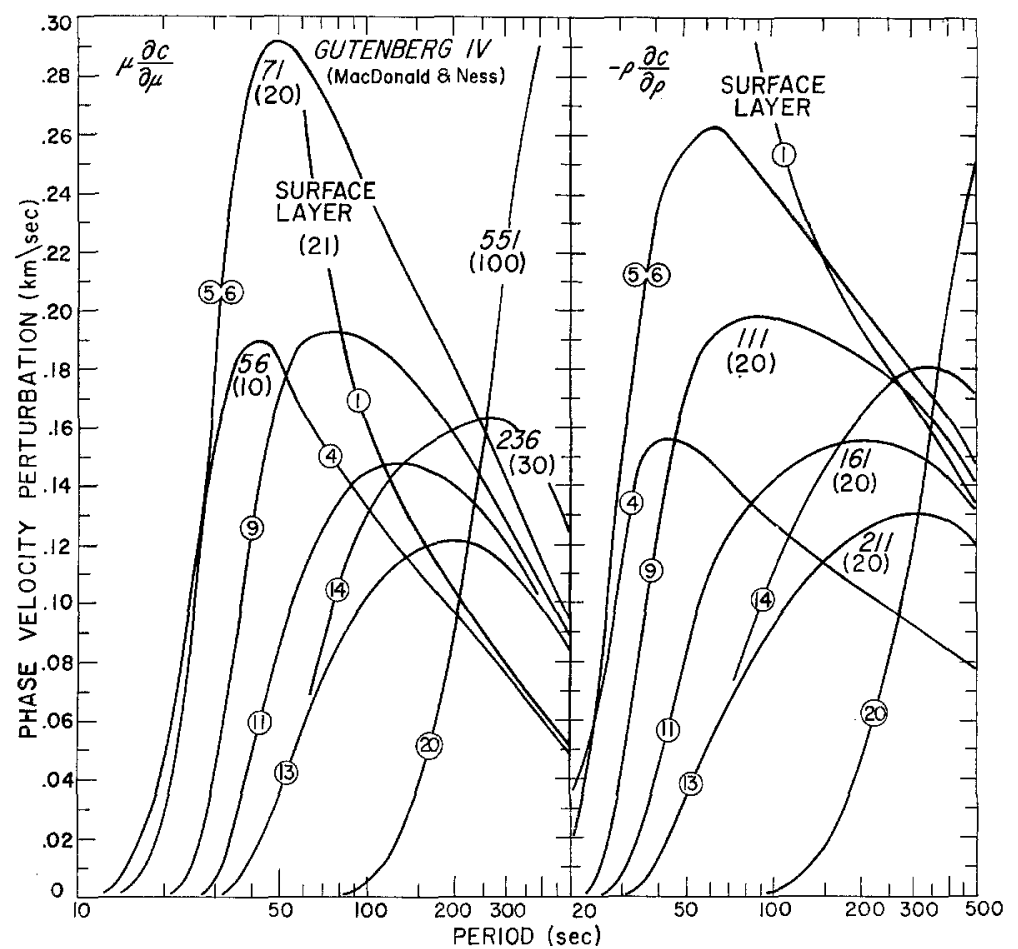

Frg. 4. Phase velocity perturbations (km/sec) for Gutenberg IV Earth Model.

case is about 23 seconds, a large amount of energy is in the low-velocity channel. At shorter periods most of the energy is in the crustal layers. The decoupling phenomenon also manifests itself by nearly vertical portions in the phase and group velocity curves.

Figures 7 and 8 show the partial derivative curves for the standard model designated CIT13F for the period range 10 to 1000 seconds. Note the double logarithmic scale. Figure 9 shows the shear velocity structure of this model and, for comparison, two other proposed oceanic models. The ticks on the ordinate show how this model was split into layers.

These curves all show the equal role played by density and rigidity in the Love wave problem. These are the natural variables in any theoretical discussion. However, the shear velocity and the density are more often the variables of interest in 


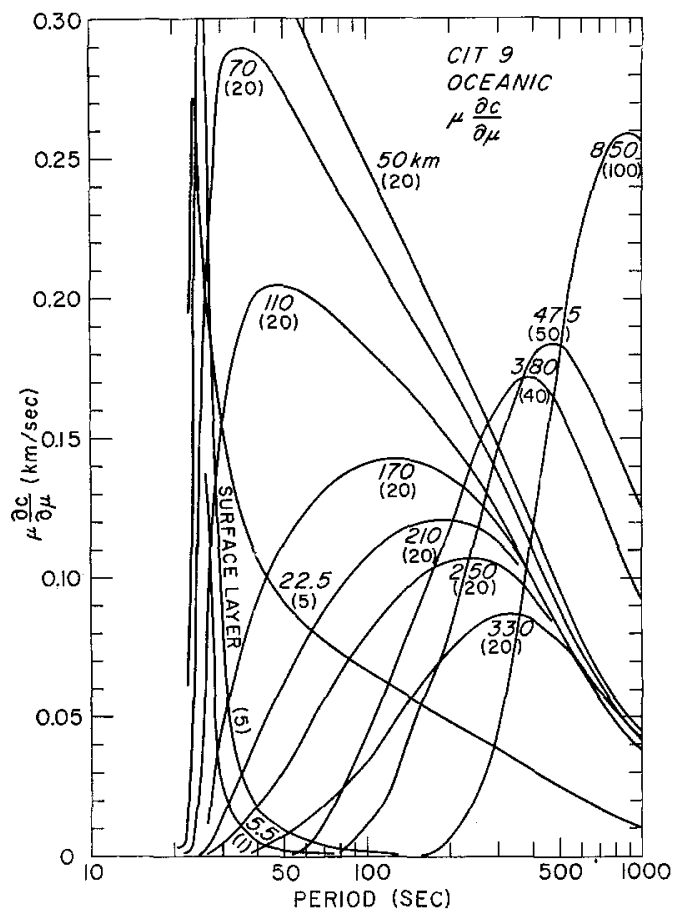

FIG. 5. Phase velocity perturbations due to a change in various layer rigidities (density held constant) for model CIT9.

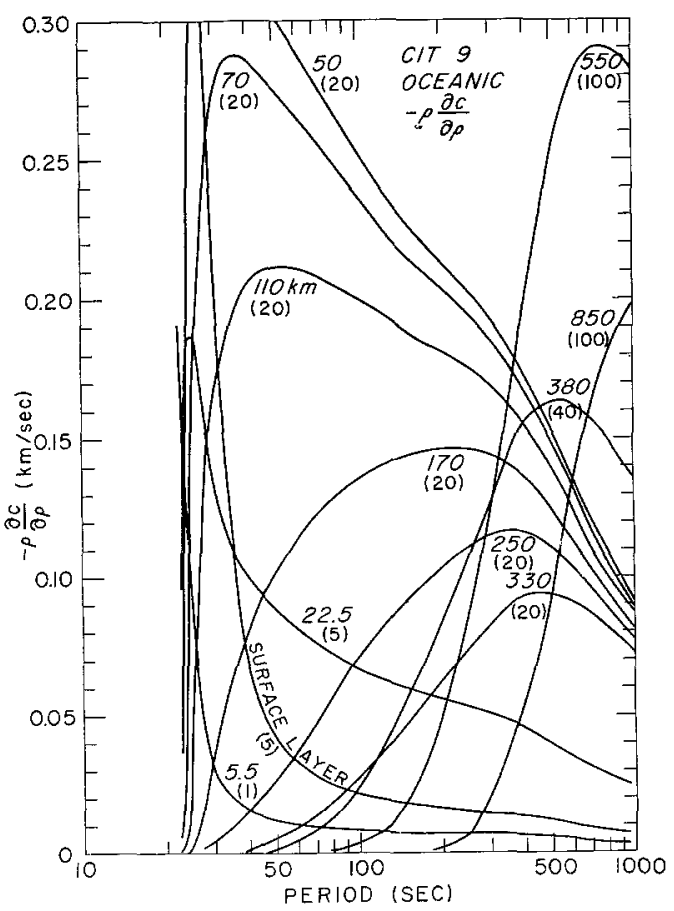

FIG. 6. Phase velocity perturbations due to a change in various layer densities (rigidity held constant) for model CIT9. 


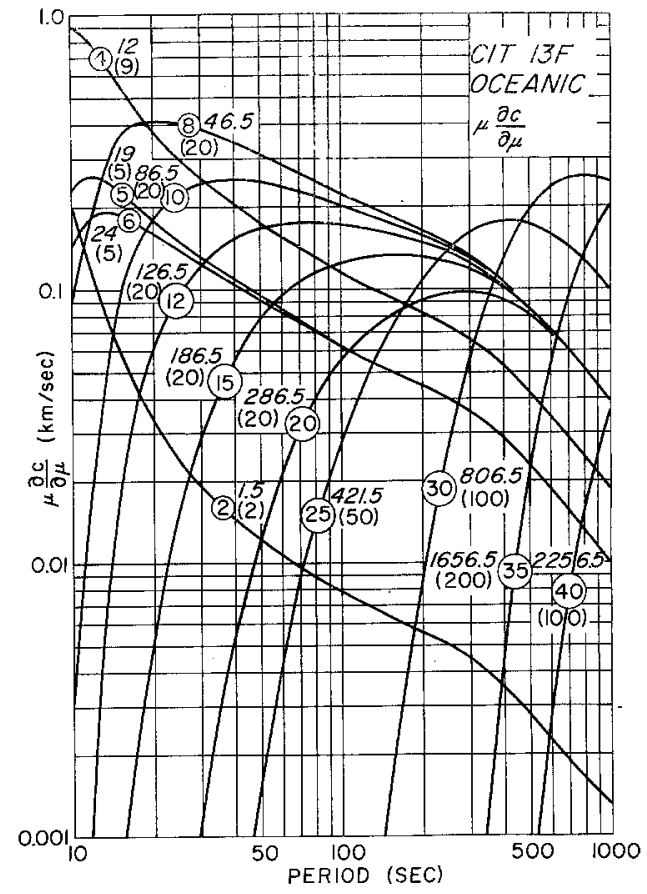

Fig. 7. Phase velocity perturbations corresponding to rigidity changes (density held constant) in various layers of the CIT13F Standard Oceanic Model. To use multiply ordinate by the fractional change in the layer rigidity being modified. The circled numbers are layer designations, italicized numbers are depths to layer midpoints, parenthetical numbers are layer thicknesses.

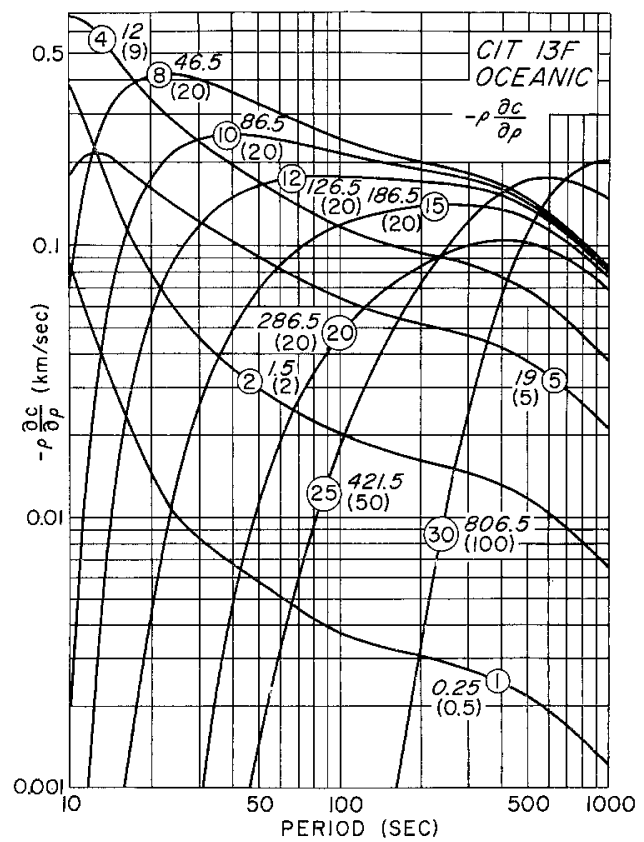

FIG. 8. Phase velocity perturbations corresponding to density changes (rigidity held constant) in various layers of the CIT13F Standard Oceanic Model. See caption of figure 7 for explanation. If the parameter perturbations are imaginery, then the curves are proportional to the resulting $\mathrm{Q}^{-1}$. 
the corresponding experimental problem since travel time results provide the starting point for most surface wave interpretations. Figure 10 shows the relative effect of shear velocity perturbations (density held constant) and density perturbations (velocity held constant) and indicates the predominant role played by the shear velocity. It is clear that the reliability of density determinations using Love wave results will be an order of magnitude less than the reliability of the shear velocity determinations. The situation improves, of course, if the shear velocity is known.

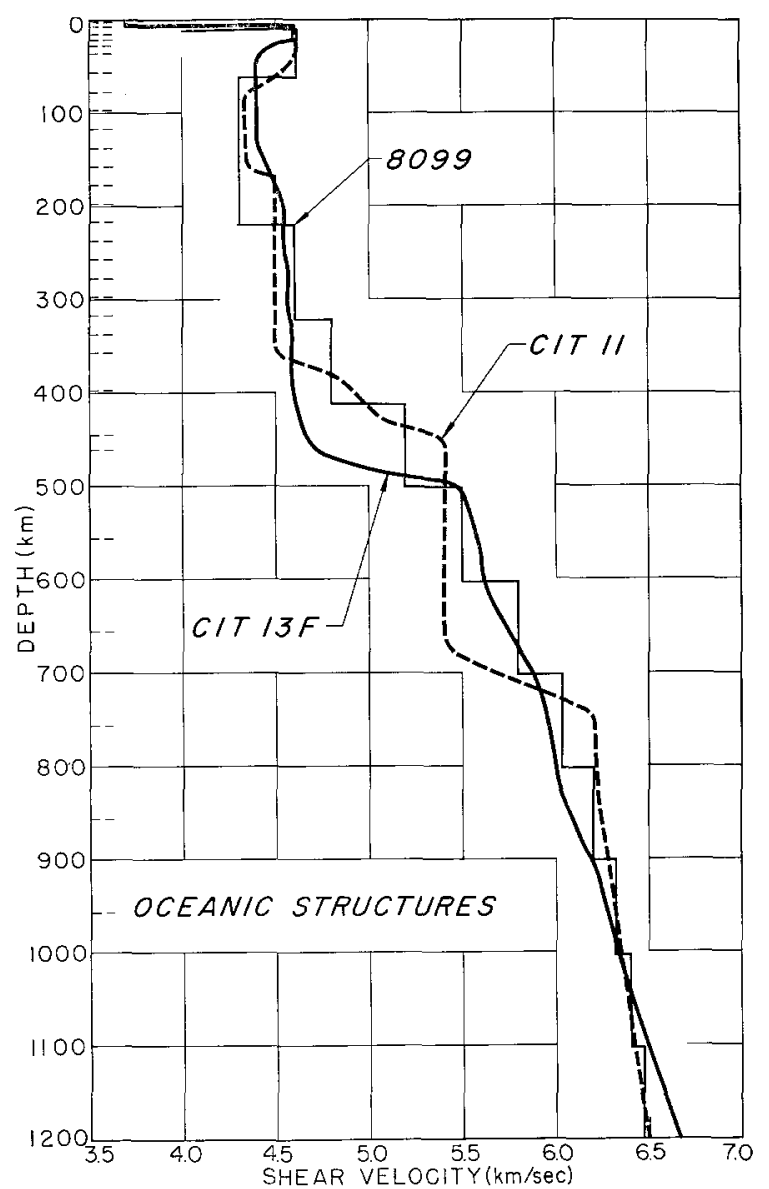

FIg. 9. Shear velocity structures for CIT13F, CIT11 and 8099.

independently. In fact, surface waves and free oscillations promise to be in important additional source of information concerning the density structure in regions of the Earth where the seismic velocities are well known.

The similarity in shape of the partial derivative curves for the various layers suggests that a considerable lack of uniqueness is involved when an attempt is made to find a linear combination of the theoretical perturbation curves that match an observed "discrepancy curve." This question is discussed more rigorously in a forthcoming paper (Archambeau and Anderson, in preparation). Ambiguity can be reduced by using higher mode data as a supplementary constraint. Phase velocity 
perturbations for the 2nd Love Mode for the Gutenberg-Birch Continental Model are shown in figure 11. Note the double peaked character of these curves compared with the single peak fundamental mode curves. Of particular interest here is the suggestion that this mode, the so-called $S a$ wave, in the period range of about 15-20 seconds, "sees" the upper mantle from about $80-150 \mathrm{~km}$. more than it sees the crustal layers, making this a particularly powerful phase for the study of the upper mantle.

The direct calculation of group velocity perturbations requires special techniques not covered in this paper. It is easy, however, to devise numerical schemes for determining a group velocity correction from derived phase velocity corrections.

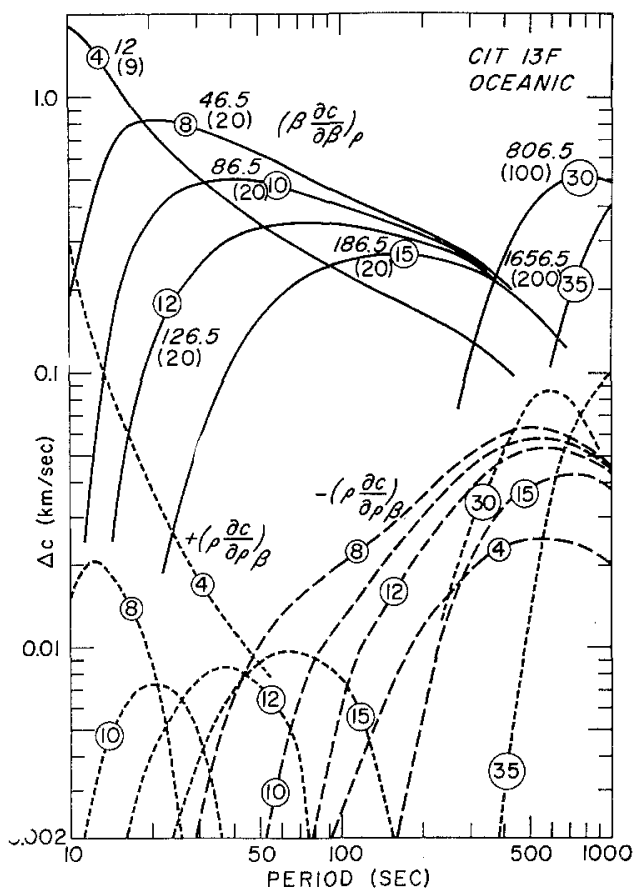

FrG. 10. Phase velocity perturbations for CIT13F Standard Oceanic Model with shear velocity and density as the independent variables.

For example, a two-point difference scheme yields

$$
U=U_{0}(1+\alpha)
$$

where

$$
\begin{aligned}
\alpha & =\left(\delta k_{-1}-\delta k_{+1}\right) /\left(k_{+1}-k_{-1}\right) \\
\delta k & =-(k \delta c / c) \\
k & =2 \pi / c T
\end{aligned}
$$


where the $\delta k$ are those determined at periods adjacent to the period for which group velocity is desired. Tables 12 and 13 provide group velocity information for the standard models.

A later paper will deal with the free oscillation problem. The question in this case is how the period $(\sigma)$ of a free oscillation of order $n$ is affected by changes in the physical properties. Meanwhile, torsional oscillation data for periods less

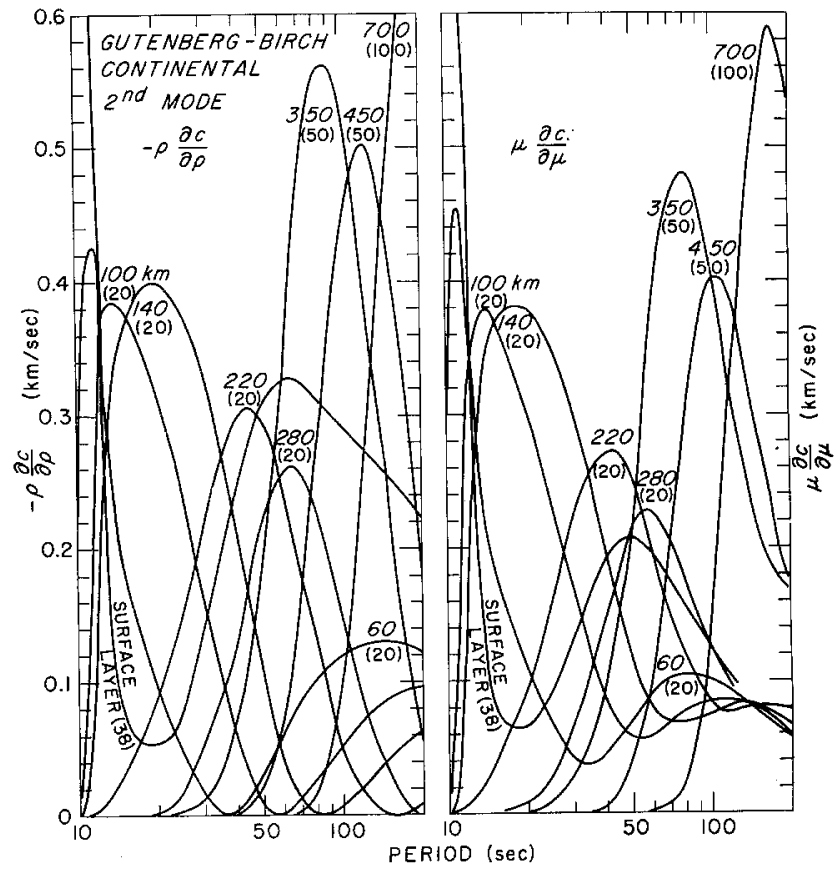

Fig. 11. Phase velocity perturbations for 1st higher Love mode for Gutenberg-Birch Continental Model. Note the "windows" that occur in certain period ranges which allow deeper layers to be seen through the nearer surface layers.

than 1000 seconds may be interpreted by converting it to dispersion data by computing

$$
\begin{aligned}
T & =2 \pi / \sigma \\
c & =2 \pi /\left[\left(n+\frac{1}{2}\right) \cdot T^{\prime}\right]
\end{aligned}
$$

and proceeding as in the travelling wave case.

\section{The Inverse Surface Wave Problem}

The usual method of interpreting surface wave data is by an indirect trial and error procedure. The tables and graphs in this paper can be used as guidelines in a technique of this sort. Dorman and Ewing (1962) systematized the trial and error method by numerically solving a simultaneous set of linear equations relating empirically determined parameter corrections to the discrepancy between the 
observed and the numerical trial dispersion data. The tables of theoretical partial derivatives presented in this paper can be used to replace the numerical derivatives in this technique. Preliminary results utilizing this approach were presented at the recent IUGG meeting (Anderson, et al. (1963), Archambeau and Anderson (1963)). Alternatively, the closed form expressions for the various partial derivatives can be used to do the same sort of thing completely analytically to avoid the numerical difficulties arising in the attempted inversion of almost singular matrices.

TABLE 12

Period $(T)$, Phase Velocity $(c)$ and Group Velocity $(U)$ for Gutenberg Birch II Continental Model

$\begin{array}{cccccc}T & \begin{array}{c}\mathrm{T} \\ (\mathrm{secs})\end{array} & \begin{array}{c}\mathrm{U} / \mathrm{km} / \mathrm{sec}) \\ (\mathrm{km} / \mathrm{sec})\end{array} & \begin{array}{c}\mathrm{T} \\ (\mathrm{secs})\end{array} & \begin{array}{c}\mathrm{c} \\ (\mathrm{km} / \mathrm{sec})\end{array} & \begin{array}{c}\mathrm{U} \\ (\mathrm{km} / \mathrm{sec})\end{array} \\ 1079.48 & 6.72 & 6.060 & 60.37 & 4.41 & 4.1240 \\ 951.57 & 6.62 & 5.783 & 56.60 & 4.39 & 4.0967 \\ 861.17 & 6.52 & 5.576 & 53.17 & 4.37 & 4.0664 \\ 788.74 & 6.42 & 5.406 & 50.07 & 4.35 & 4.0339 \\ 673.13 & 6.22 & 5.131 & 47.28 & 4.33 & 3.9994 \\ 580.00 & 6.02 & 4.912 & 44.78 & 4.31 & 3.9637 \\ 500.19 & 5.82 & 4.734 & 42.52 & 4.29 & 3.9273 \\ 422.12 & 5.60 & 4.5792 & 40.47 & 4.27 & 3.8909 \\ 388.89 & 5.50 & 4.5220 & 38.62 & 4.25 & 3.8549 \\ 356.64 & 5.40 & 4.4727 & 36.92 & 4.23 & 3.8198 \\ 325.10 & 5.30 & 4.4307 & 35.36 & 4.21 & 3.7860 \\ 294.04 & 5.20 & 4.3955 & 33.91 & 4.19 & 3.7537 \\ 263.25 & 5.10 & 4.3664 & 32.57 & 4.17 & 3.7231 \\ 232.54 & 5.00 & 4.3424 & 31.31 & 4.15 & 3.6944 \\ 201.79 & 4.90 & 4.3222 & 30.12 & 4.13 & 3.6678 \\ 170.93 & 4.80 & 4.3039 & 28.99 & 4.11 & 3.6431 \\ 155.49 & 4.75 & 4.2946 & 27.91 & 4.09 & 3.6204 \\ 140.07 & 4.70 & 4.2847 & 26.88 & 4.07 & 3.5998 \\ 124.77 & 4.65 & 4.2732 & 25.89 & 4.05 & 3.5812 \\ 109.70 & 4.60 & 4.2588 & 24.93 & 4.03 & 3.5645 \\ 106.74 & 4.59 & 4.2554 & 23.99 & 4.01 & 3.5497 \\ 100.87 & 4.57 & 4.2479 & 23.53 & 4.00 & 3.5430 \\ 95.10 & 4.55 & 4.2393 & 21.30 & 3.95 & 3.5161 \\ 89.47 & 4.53 & 4.2293 & 19.14 & 3.90 & 3.4993 \\ 84.00 & 4.51 & 4.2177 & 17.00 & 3.85 & 3.4916 \\ 81.33 & 4.50 & 4.2111 & 14.83 & 3.80 & 3.4917 \\ 78.73 & 4.49 & 4.2041 & 12.56 & 3.75 & 3.4982 \\ 73.70 & 4.47 & 4.1883 & 3.50 & 3.65 & 3.5089 \\ 68.94 & 4.45 & 4.1698 & 3.5089 & 3.5309 \\ 64.49 & 4.43 & 4.1484 & & & \end{array}$

\section{ACKNOWLEDGMENTS}

The author wishes to express his appreciation to Dr. David G. Harkrider, M. N. Toksöz and Charles B. Archambeau for many helpful discussions. Laszlo Lenches was of great assistance in the preparation of the manuscript.

This research was supported by AF-AFOSR-25-63 of the Air Force Office of Scientific Research as part of the Advanced Research Projects Agency, project VELA. 
TABLE 13

Period ( $T$ ), Phase Velocity $(c)$, and Group Velocity $(U)$ for Cit13F Oceanic Model

$\begin{array}{ccc}T & c & \\ (\text { secs }) & (\mathrm{km} / \mathrm{sec}) & \mathrm{U} \\ 1009.06 & 6.72 & 5.96 \\ 899.29 & 6.62 & 5.71 \\ 818.64 & 6.52 & 5.52 \\ 696.84 & 6.32 & 5.216 \\ 602.14 & 6.12 & 4.978 \\ 522.28 & 5.92 & 4.787 \\ 451.55 & 5.72 & 4.635 \\ 412.00 & 5.60 & 4.561 \\ 380.15 & 5.50 & 4.509 \\ 318.19 & 5.30 & 4.433 \\ 287.45 & 5.20 & 4.4093 \\ 256.41 & 5.10 & 4.3944 \\ 224.67 & 5.00 & 4.3886 \\ 191.71 & 4.90 & 4.3915 \\ 156.82 & 4.80 & 4.4020 \\ 119.10 & 4.70 & 4.4162 \\ 98.96 & 4.65 & 4.4211 \\ 78.14 & 4.60 & 4.4206 \\ 57.53 & 4.55 & 4.4109 \\ 38.58 & 4.50 & 4.3927 \\ 23.42 & 4.45 & 4.354 \\ 15.56 & 4.40 & 4.2349 \\ 12.55 & 4.35 & 4.0593 \\ 11.00 & 4.30 & 3.889 \\ 9.23 & 4.20 & 3.605 \\ 7.29 & 4.00 & 3.2067 \\ 6.32 & 3.85 & 2.9920 \\ 4.05 & 3.30 & 2.281\end{array}$

\section{REFERENCES}

Anderson, Don L.

1961. "Elastic Wave Propagation in Layered Anisotropic Media," J. Geophys. Research, $66,2593-2963$.

1962. "Love Wave Dispersion in Heterogeneous Anisotropic Media," Geophysics, 27, 445454.

Anderson, Don L. and M. N. Toksöz

1963. "Surface Waves on a Spherical Earth," J. Geophys. Research, 68, 3483-3500.

Anderson, Don L., M. N. Toksöz and R. Kovach

1963. "Upper Mantle Structure from Long Period Surface Waves," I.U.G.G. Upper Mantle Symposium, XIII General Assembly, Berkeley.

Archambeau, C. B. and Don L. Anderson

1963. "Inversion of Surface Wave Dispersion Data," I.U.G.G., XIII General Assembly, Berkeley.

Dorman, V. and M. Ewing

1962. "Numerical Inversion of Seismic Surface Wave Dispersion Data and Crust-Mantle Structure in the New York-Pennsylvania Area," J. Geophys. Research, 67, 5227-5242.

Gilbert, F. and G. J. F. MacDonald

1960. "Free Oscillations of the Earth, I. Toroidal Oscillations," J. Geophys. Research, $65,675-693$. 
Harkrider, D. G. and Don L. Anderson

1962. "Computation of Surface Wave Dispersion for Multilayered Anisotropic Media," Bull Seism. Soc. Am., 52, 321-332.

Haskell, N. A.

1953. "Dispersion of Surface Waves on Multi-layered Media," Bull Seism. Soc. Am., 43, $17-34$.

Jeffreys, H.

1961. "Small Corrections in the Theory of Surface Waves," Geophys. Journal, 6, 115-117.

MacDonald, G. J. F. and N. F. Ness

1961. "A Study of the Free Oscillations of the Earth," J. Geophys. Research, 66, 1865-1912.

SeIsmological Laboratory

California Institute of Technology

Pasadena, California

Diviston of the Geological Sciences

Contribution No. 1222

Manuscript received December 6, 1963. 